\title{
Metallization and Charge-Transfer Gap Closure of Transition-Metal Iodides under Pressure
}

by

Anthony Li-Chung Chen

Ph. D. Thesis

Department of Physics

UNIVERSITY of CALIFORNIA at BERKELEY

and

Materials Sciences Division

\section{LAWRENCE BERKELEY LABORATORY}

University of California

Berkeley, CA 94720

May 1993

This work is supported by the Director, Office of Energy Research, Office of Basic Energy Sciences, Materials Sciences Division, of the U.S. Department of Energy under Contract No. DE-AC03-76SF00098

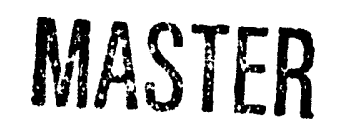




\section{Table of Contents}

Dedication

Page

Table of Contents

iii

List of Figures and Tables

iv

Acknowledgments

vi

I. Introduction to Transition-Metal Compounds and

viii

Insulator-Metal Transitions

II. $\mathrm{NiI}_{2}, \mathrm{CoI}_{2}$, and $\mathrm{FeI}_{2}$ : Sample Properties and Crystal Growth

A: Crystal Structure and F ectronic Properties 6

B: Crystal Growth 9

III. Theoretical Considerations of Band Overlap Insulator-Metal . 17

Transitions and Magnetic Transitions

A: Insulator-Metal Transitions and Band Overlap 17

B: Magnetic Transitions 20

IV. Measurements Under High Pressure: Experimental Procedure 28

A: Resistivity Measurements under High Pressure 28

B: Near-IR Absorption Measurements under $\quad 32$

High Pressure

V. High Pressure Mössbauer and X-ray Diffraction Studies

of $\mathrm{NiI}_{2}$ and $\mathrm{CoI}_{2}:$ Discovery of an Isostructural

Magnetic Transition

VI. Resistivity Data and Analysis

A: Resistivity Data of $\mathrm{NiI}_{2} \quad 52$

B: Resistivity Data of $\mathrm{CoI}_{2}$ and $\mathrm{FeI}_{2}$

VII. Absorption Data and Analysis 66

A: Absorption Data of $\mathrm{NiI}_{2} \quad 66$ 
B: Absorption Data of $\mathrm{CoI}_{2}$ and $\mathrm{FeI}_{2} \quad 68$

$\begin{array}{ll}\text { VIII. Discussion } & 78\end{array}$

A: Origin of the $\mathrm{IM}$ Transitions and Its Relation $\quad 78$

to the Magnetic Transitions in the TMIs

B: Discussion of the Near-IR Absorption Data: 81

Metallization by Charge-Transfer Gap Closure

C: Evidence of a First-Order Insulator-Metal Transition 83

IX. Conclusion and Future Efforts $\quad 89$

References $\quad 92$

Appendix: Pressure Dependence of $d-d$ Transitions 95

in $\mathrm{NiI}_{2}$ and $\mathrm{CoI}_{2}$ 


\section{List of Figures}

Figure Number ana Description

2.1 Crystal structures of $\mathrm{NiI}_{2}, \mathrm{CoI}_{2}$, and $\mathrm{FeI}_{2}$. 13

2.2 Probable density of states of the transition-metal iodides. 14

2.3 Band structure calculation of $\mathrm{NiBr}_{2}$. 15

2.4 Optical and Near-IR absorption spectra of $\mathrm{NiCl}_{2}$ and $\mathrm{NiBr}_{2}$. 16

3.1 Band overlap metallization. 24

3.2 Charge carrier density as a function of energy gap. 25

$\begin{array}{lll}3.3 & \text { Phase diagram of } \mathrm{NiI}_{2} \text {. } & 26\end{array}$

3.4 Zero-temperature phases of $\mathrm{NiI}_{2}$. 27

4.1 Schematic drawing of a diamond anvil cell. 37

4.2 Gasket preparation for resistivity measurements. 38

4.3 Wiring of the diamond anvil cell for resistivity measurements. 39

4.4 Experimental setup for absorption measurements. 40

5.1 Mössbauer spectra of $\mathrm{NiI}_{2}$ under pressure. $\quad 46$

5.2 Néel temperature, hyperfine magnetic field, and isomer shift of 47 $\mathrm{NiI}_{2}$ under pressure.

5.3 Mössbauer spectra of $\mathrm{CoI}_{2}$ under pressure. $\quad 48$

5.4 Néel temperature, hyperfine magnetic field, and isomer shift of 49 $\mathrm{CoI}_{2}$ under pressure.

5.5 Volume vs. pressure data of $\mathrm{NiI}_{2}$. 50

$5.6 \quad \mathrm{c}$ and a lattice constants of $\mathrm{NiI}_{2}$ under pressure. 51

6.1 Quasi-four-probe resistance vs. pressure data of $\mathrm{NiI}_{2}$. 60

6.2 True four-probe resistivity vs. pressure data of $\mathrm{NiI}_{2}$. 61

6.3 Arrhenius plots of $\mathrm{NiI}_{2}$ at various pressures. 62

6.4 Activation energy vs. pressure for $\mathrm{NiI}_{2}$. 63

6.5 Resistivity vs. pressure data of $\mathrm{CoI}_{2}$. 64 
6.6 Resistivity vs. pressure data of $\mathrm{FeI}_{2}$. 65

$7.1 \mathrm{NiI}_{2}$ near-IR absorption data. $\quad 72$

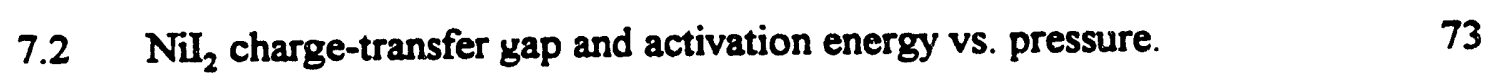

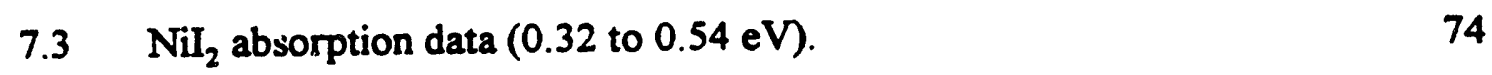

$7.4 \mathrm{CoI}_{2}$ near-IR absorption data. $\quad 75$

$7.5 \mathrm{FeI}_{2}$ near-IR absorption data 76

$7.6 \mathrm{CoI}_{2}$ and $\mathrm{FeI}_{2}$ charge-transfer gap vs. pressure. $\quad 77$

8.1 Scenarios for band overlap metallization. 88

Al Proposed symmetry of the crystal field of the transition 101 metal halides under pressure.

A2 Splitting of the $d$ levels of the $\mathrm{Ni}^{2+}$ and $\mathrm{Co}^{2+}$ ions in $\mathrm{NiI}_{2}$ and $\mathrm{CoI}_{2}$. 102

A3 Calculated crossings and anticrossings of the near-IR intra-3d 103 transitions in $\mathrm{NiI}_{2}$.

A4 Calculated crossings and anticrossings of the near-IR intra-3d $\quad 104$ transitions in $\mathrm{CoI}_{2}$.

A5 Peak positions of the intra-3d transitions as a function of 105 pressure for $\mathrm{NiI}_{2}$ and $\mathrm{CoI}_{2}$.

\section{List of Tables}

2.1 Ambient pressure properties of $\mathrm{NiI}_{2}, \mathrm{CoI}_{2}$, and $\mathrm{FeI}_{2}$. 11

9.1 Summary of the properties of $\mathrm{NiI}_{2}, \mathrm{CoI}_{2}$, and $\mathrm{FeI}_{2}$ determined by this work. 


\section{Chapter I : Introduction to Transition-Metal Compounds and Insulator-Metal Transitions}

The electronic structure of all crystalline materials can be conveniently divided into two general categories. At one extreme are the materials whose electrons have extended periodic wavefunctions in the form of Bloch waves. The alkali metals are prime examples. Their electronic properties, such as excitation spectra and electrical conductivity, can be aptly described using a picture involving single-electron processes. As a resuit their bulk properties are well understood from a theoretical standpoint and can be accurately determined from first-principles bandstructure calculations. At the other extreme are the materials whose electronic properties are strongly influenced by localized electrons. The magnetic transition-metal compounds fall into this regime. Magnetic ground states and hopping conductivity often characterize such materials. Our fundamental understanding of such systems is much poorer by comparison; even their ground-state properties are difficult to predict from first principles.

Sir Nevill Mott did much of the ground breaking work in our understanding of highly localized electron systems [1,2]. He first proposed the existence of a solid, now called a Mott insulator, that is an insulator because its electrons are highly correlated. High electron correlation results from the localized nature of the wavefunctions. Suppose one naively considers a system with an odd number of valence electrons per unit cell. If one neglects electron-electron interaction, the prototype solid is metallic since one of the bands is certain to be partially filled. However, if the electron wavefunctions are highly localized on the atomic sites, as in the case of the $d$ and $f$ shell electrons in the transition metals and rare earths respectively, the electron-electron interaction cannot be ignored. It costs a great deal of energy to move an electron from one ion to another because of the strong onsite Coulomb repulsion from the electrons on the other ion. This repulsion produces an effective energy gap to conduction known as the Mott-Hubbard gap (U) [3]. These systems are said to have highly correlated electrons because the electrons prefer to 
distance themselves from each other by remaining on their own atomic site. Many of the transition-metal compounds such as $\mathrm{NiO}$ are examples of compounds with large $U$ making them prime candidates for Mott insulators [4-5].

In a Mott insulator, the electronic wavefinnctions may transform from localized to extended states with variation of some thermodynamic variable such as pressure or temperature. One can visualize, for example, how this transition can result from application of hydrostatic pressure since pressure will decrease the lattice constants of the material. This leads to an increase in the overlap of adjacent localized wavefunctions and eventually produces a single-electron band. Mott again was the first to develop the theory of this eponymous transition [1,2]. As the wavefunctions become extended, Mott argued that the system should experience an insulator-metal transition (IM) with a simultaneous disappearance of magnetic moments. The search for Mott transitions is complicated by the existence of other conductivity gaps in real systems and crystal distortions that usually accompany such transitions. Few of the $\mathrm{MM}$ transitions observed in the transition-metal compounds are believed to be true Mott transitions as originally envisioned by Mott. In many cases the wavefunctions in question are already extended and merely overlap with another band. The temperature-driven $\mathrm{MM}$ transitions in $\mathrm{V}_{2} \mathrm{O}_{3}$ and $\mathrm{VO}_{2}$ are two notable exceptions $[6,7]$.

Recently our understanding of Mott insulators has been drastically changed by a new theory of the transition-metal compounds proposed by Zaanen, Sawatzky, and Allen (ZSA) [8]. In 1985, ZSA proposed a more general model which accounted for the large variation of insulating and metallic behaviors among the transition-metal compounds. They pointed out that a large $U$ is only a necessary condition for obtaining a finite transport gap. In their framework, there is a second kind of insulators in which the charge-transfer energy $(\Delta)$ rather than the Mott gap is the minimum transport gap. This $\Delta$ is the energy necessary to excite an electron from the anion valence band to the 
transition-metal ion. More formally $U$ and $\Delta$ are defined as the energies required to make following transitions in a transition-metal compound.

$$
\begin{array}{ll}
\Delta: & d^{h} \rightarrow d^{h+1} L \\
U: & d^{n} d^{h} \rightarrow d^{n-1} d^{h+1}
\end{array}
$$

Here $L$ represents a ligand hole, such as a hole in the anion valence $p$ band. The electronic properties of a transition-metal compound are determined by the relative magnitudes of these two energies. According to ZSA there are four possible different cases, which listed below.
1) $\Delta>U>0$
Mott Insulator
2) $U>\Delta>0$
Charge-Transfer Insulator
3) $U=0, \Delta>0$
Mott Metal
4) $\Delta=0, U>0$
Charge-Transfer Metal

Based on x-ray photoemission, which is able to measure both $U$ and $\Delta$, Sawatzky and Allen concluded that nickel oxide is a charge-transfer insulator rather than a Mott insulator as previously believed [4]. A similar study of the nickel dihalides also showed them to be charge-transfer insulators [9]. More recent reports of $\mathbf{M}$ transitions in transition-metal compounds have been interpreted along the lines of the ZSA framework $[10,11]$.

The introduction of a new kind of insulator opens the possibility for a new type of $\mathrm{M}$ transition that results from the closure of the charge-transfer gap. To date this type of transition has only been reported [11] in some perovskites of the formula $R \mathrm{NiO}_{3}$ where $R$ is a rare-earth ion. This type of transition was previously undocumented, and what changes in the magnetic and optical properties that accompany the $\mathbf{M}$ transition were unknown. We investigated for the first time in this thesis the possibility of inducing this type of transition in $\mathrm{NiI}_{2}, \mathrm{CoI}_{2}$, and $\mathrm{FeI}_{2}$ by application of hydrostatic pressure. These crystals are highly compressible so large changes in their properties are expected with application of moderately high pressures. Our efforts have led to the discovery of a pressure-induced $\mathrm{IM}$ transition in $\mathrm{NiI}_{2}$ that simultaneously shows an antiferromagnetic to 
diamagnetic transition due to disappearance of the Ni magnetic moments. An x-ray study showed that there was no change in crystal structure across the transition. Initially it was uncertain whether this IM phase transition was a Mott or a charge-transfer type. Both are expected to be accompanied by disappearance of magnetic moments but for fundamentally different reasons. Since then several theoretical studies of this transition have assumed that the charge-transfer transition is the underlying cause. By comparing IR-absorption and resistivity measurements, we show that the charge-transfer gap decreases under pressure and that closing of this gap is the fundamental cause of the $\mathbf{I M}$ transition in $\mathrm{NiI}_{2}$. In addition we found evidence for a discontinuous gap closure suggesting that the transition is first-order. Similar absorption and resistivity measurements on $\mathrm{CoI}_{2}$ and $\mathrm{FeI}_{2}$ show that the entire family of the late transition-metal iodides (TMI) metallize under pressure by charge-transfer gap closure.

Additional motivation for this work stems from the current interest in the highpressure physics community in pressure-induced metallization. It has been realized very early on that every solid will eventually metallize under sufficiently high pressure. Through much work it has now been shown that but the route from insulating to metallic behavior is rich and varied. Some IM transitions are the result of structural transformations (e.g. silicon [12]) while others are due to isostructural overlap between valence and conduction bands (e.g. xenon [13] and hydrogen [14]). However, so far no discontinuities in the closing energy gap have been reported in all of the band-overlap transitions at high pressures. Our measurements of the transition-metal iodides are the first experimental evidence that band-overlap transitions in these materials can be firstorder, as suggested by theory.

The remainder of this thesis is organized in the following way. Chapter II consists of a description of the ambient pressure properties of th. TMIs and a description of the method for growing the crystals. In Chapter III we summarize the present understanding of insulator-metal transitions in general and specifically in these compounds. Chapter IV 
is a detailed account of the experimental procedures that we followed to prepare a diamond-anvil cell for resistivity and absorption measurements and to measure these properties under pressure. Mössbauer and $x$-ray data showing a pressure induced, isostructural magnetic transition in $\mathrm{NiI}_{2}$ and $\mathrm{CoI}_{2}$ are presented in Chapter V. Chapter VI and VII are devoted, respectively, to the resistivity and IR-absorption results in the TMIs. Chapter VIII contains the conclusions based on these results and their implication. Chapter $\mathbf{X}$ is a summary of the entire thesis. As part of the $\mathbb{R}$ measurements we have also studied the pressure dependence of the intra-ion $3 d$ transitions in $\mathrm{NiI}_{2}$ and $\mathrm{CoI}_{2}$. Since the results are not relevant to the main theme of this thesis, they are presented in the appendix. 


\section{Chapter II: $\mathrm{NiI}_{2}, \mathrm{CoI}_{2}$, and $\mathrm{FeI}_{2}$ : Sample Properties and Crystal Growth}

\section{A: Drystal Structure and Electronic Properties}

$\mathrm{NiI}_{2}, \mathrm{CoI}_{2}$, and $\mathrm{FeI}_{2}$ all crystallize in similar hexagonal layered structures [15]. These crystal structures are easily visualized as formed by a hexagonal layer of the transition-metal ions sandwiched between two hexagonal layers of iodine ions. This "triple slab" is then repeated and stacked on top of each other with the planes perpendicular to the c-axis. The difference between the three compounds is the sequence of the stacking. Both $\mathrm{CoI}_{2}$ and $\mathrm{FeI}_{2}$ have the $\mathrm{CdI}_{2}$ structure in which the stacking sequence is $. . A C B, A C B, A C B \ldots$ while $\mathrm{NiI}_{2}$ crystallizes in the $\mathrm{CdCl}_{2}$ structure with stacking sequence ..ACB, $C B A, B A C, A C B \ldots$ In this notation the commas separate each triple slab. Both structures are illustrated in Fig. 2.1. The space group of $\mathrm{NiI}_{2}$ is $D_{3 d}^{\mathrm{s}}$ whereas that of $\mathrm{CoI}_{2}$ and $\mathrm{FeI}_{2}$ are $D_{3 d}^{3}$.

In the TMIs, the $3 d$ electrons of the transition-metal ions remain in their elemental $3 d^{\text {h }}$ configuration. In the solid a gap forms between occupied and unoccupied $3 d$ states because of strong electron-electron interaction. These electronic states thus do not contribute to conductivity even though they are partially filled. They are more accurately treated as localized impurity states that are split by the crystal field of their nearestneighbor iodine ions. The nearest-neighbor crystal field is approximately cubic with a small trigonal distortion [16]. Neglecting the spin-orbit coupling, the representations of the ground-state $3 d$ electrons of $\mathrm{NiI}_{2}, \mathrm{CoI}_{2}$, and $\mathrm{FeI}_{2}$ within the $D_{3 \mathrm{~d}}$ point group are ${ }^{3} A_{2 \mathrm{~g}}$, ${ }^{4} T_{1 \mathrm{~g}},{ }^{5} E_{2 \mathrm{~g}}$ respectively [17].

Because their $3 d$ shells are partially filled, the transition-metal ions have magnetic moments. In $\mathrm{NiI}_{2}, \mathrm{CoI}_{2}$, and $\mathrm{FeI}_{2}$ the $3 d$ shells have total spins $S=1, S=3 / 2$, and $S=2$ respectively [18]. The magnetic moments of the ions couple ferromagnetically within the hexagonal planes and antiferromagnetically between the planes. The crystals are 
antiferromagnetic at low temperatures with Néel temperatures $\left(T_{\mathrm{N}}\right)$ of $75 \mathrm{~K}, 3-12 \mathrm{~K}$, and $10 \mathrm{~K}$ [18] respectively. In addition, at lower temperatures the spins in $\mathrm{NiI}_{2}$ and $\mathrm{CoI}_{2}$ are known to form helical structures [19].

The TMIs are excellent insulators at atmospheric pressure [20]. In forming the iodides, the transition-metal ions give up their two $4 s$ electrons to fill the iodine $5 p$ shell resulting in a predominantly ionic bond. In the solid the empty $4 s$ states are expected to form a high-lying conduction band, while the iodine $5 p$ states comprise the main valence band. The Fermi level lies in between the two. The $3 d$ states are highly correlated and form an upper and lower Hubbard band with little dispersion. In an energy diagram they are typically shown schematically as two narrow bands separated by an energy, $U$. In the intrinsic material the large $U$ inhibits the hopping of a $3 d$ electron from site to site and suppresses conduction. We remind the reader that the energy of an electron in the conduction band represents the excess energy of the solid after an extra electron has been added while a valence band represents the decrease in energy of the solid after an electron has been removed. To add an electron to the upper Hubbard band of $\mathrm{NiI}_{2}$, which would be localized on a $\mathrm{Ni}^{2+}$ ion, would require at least the onsite Coulomb repulsion energy $(U)$.

Based on the arguments made in the preceding paragraph, we draw, in Fig. 2.2, a qualitative view of the density of states of these solids illustrating the relevant energy bands and gaps. Unlike ZSA, we take the more lucid approach of Mott and make a clear distinction between the charge-transfer energy $(\Delta)$ and the charge-transfer gap $\left(E_{\mathrm{ctG}}\right)$, which we defined as

$$
E_{\text {CTG }}=\Delta-\left(B_{\mathrm{v}} / 2\right)-\left(B_{\mathrm{p}} / 2\right) \text {. }
$$

Here $B_{\mathrm{U}}$ and $B_{\mathrm{p}}$ are the bandwidths of the upper Hubbard band and the anion $p$ band respectively. Similarly we define the Mott-Hubbard gap $\left(E_{\mathrm{MHG}}\right)$ as

$$
E_{\mathrm{MHG}}=U-\left(B_{\mathrm{U}} / 2\right)-\left(B_{\mathrm{L}} / 2\right),
$$


where $B_{\mathrm{L}}$ represent the bandwidth of the lower Hubbard bands. These energies and gaps are illustrated in Fig. 2.2.

Although, no band-structure calculations of any of the TMIs have been attempted, a one-electron band structure (f isostructural $\mathrm{NiBr}_{2}$ (Fig. 2.3) is available and is expected to be ciualitatively similar [21]. The main features are a manifold of valence bands about 5 eV wide composed predominantly of the anion $p$ shell, and a transition-metal 45 conduction band with a bandwidth of about $2 \mathrm{eV}$. We incorporate both features into our density-of-states figure. In addition, the calculations show a narrow band (about $0.5 \mathrm{eV}$ wide) of $3 d$ states near the Fermi level. The authors admit that their calculations are based on a single-particle approach and therefore cannot accurately treat the localized $3 d$ electrons. Eased on the one-electron calculation the Fermi level lies in the partially filled $3 d$ band so that $\mathrm{NiBr}_{2}$ should be a metal. The calculated $p$ band width in $\mathrm{NiBr}_{2}$ agrees with the result of a recent photoemission study of $\mathrm{NiI}_{2}$ [22]. The failure of the oneelectron band structure in explaining the insulating ground state of the TMIs shows clearly the importance of electron-electron correlation in these materials.

The relative positions of the bands near the Fermi level determine which kind of insulator these compounds are. Either the minimum gap lies between the I $5 p$ band and the upper Hubbard band (charge-transfer insulator) or between the lower and upper Hubbard bands (Mott insulator). From the one-electron band-structure calculation alone it is not possible to determine the nature of the minimum energy gap. There are a number of works indicating that the TMIs are charge-transfer insulators. In one reflectivity study [23] of the TMIs the authors loosely claim that the lowest energy structures are due to charge-transfer transitions or excitons associated with such transitions. The excitonic peaks are broad (FWHM $0.7 \mathrm{eV}$ ) and are centered at $\sim 2,2.5$, and $3 \mathrm{eV}$ for $\mathrm{NiI}_{2}, \mathrm{CoI}_{2}$, and $\mathrm{FeI}_{2}$ respectively. The corresponding charge-transfer continua are centered at 4 to 5 eV. From these studies we deduce that the charge-transfer gap. $\left(E_{\mathrm{ctG}}\right)$ forms the minimum gap in these crystals and $\Delta$ ranges from 4 to $5 \mathrm{eV}$ in the sequence of $\mathrm{NiI}_{2}, \mathrm{CoI}_{2}$, and $\mathrm{FeI}_{2}$. 
This conclusion is consistent with the trend one observes in the absorption spectra within the series of $\mathrm{Ni}$ dihalides in which the halogen is varied from $\mathrm{Cl}$ to $\mathrm{I}[24,25]$. As Fig. 2.4 illustrates for $\mathrm{NiCl}_{2}$ and $\mathrm{NiBr}_{2}$, these spectra show peaks caused by $\mathrm{N}^{2+}$ intra-d transitions and an absorption edge. While the peaks associated with the intra-d transitions are unaffected by changing the halogen atoms, the charge-transfer edge shifts quite drastically from $4 \mathrm{eV}$ in $\mathrm{NiCl}_{2}$ to $1.2 \mathrm{eV}$ in $\mathrm{NiI}_{2}$. The edge of the charge-transfer transition is expected to show just such a shift because of decrease in the anion electron affinity from $\mathrm{Cl}$ to I.

$U$ cannot be determined from the optical data because the oscillator strength of the transition is weak. Photoemission, however, can measure both $U$ and $\Delta$ as sidebands of a deep core-level emission of the transition-metal ion $[4,9]$. From such a study of $\mathrm{NiI}_{2}$ Zaanen et. al. deduced that $U=4.5 \mathrm{eV}$ and $\Delta=1.5 \mathrm{eV}$ and hence that $\mathrm{NiI}_{2}$ is a chargetransfer insulator. This value of $\Delta$ is lower than the value of $\sim 4 \mathrm{eV}$ obtained by the reflectivity measurements because the photoemission study most likely measures the gap $E_{\text {cto }}$ or its associated exciton instead. For $\mathrm{NiI}_{2}$ the associated excitons determined by the reflectivity study are about $2.0 \mathrm{eV}$, in good agreement with the photoemission study. Similarly, the value of $U$ determined by photoemission is also probably a better estimate of the gap, $E_{\mathrm{MHG}}$, or its associated exciton.

Table 2.1 summarizes the ambient pressure properties of the TMIs.

\section{B: Crystal Growth}

Detailed procedures for the growth of large platelets of the samples can be found in the literature [23] Here we outline the necessary steps. We grew the crystals by the vapor transport method inside an evacuated quartz ampoule using a three-zone furnace. The TMI compounds were obtained commercially from Alfa products in the form of high purity, fine grain powder. To grow the crystals, several grams of the starting material were placed in the bottom of a clean quartz tube with one end sealed. Care was taken to 
make certain that none of the TMI powder contaminatw the inside, growth surface of the tube and to serve as seeds for crystal growth. The tube was immediately connected to a turbomolecular vacuum pump and evacuated. This was done as soon as possible to

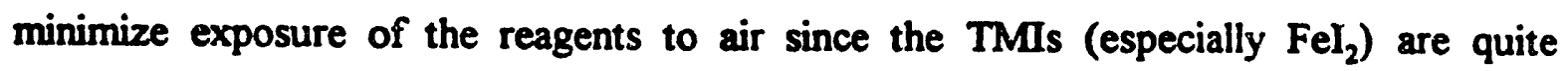
hygroscopic. The tube was then heated to $150^{\circ} \mathrm{C}$ for several hours to drive off any residual water and afterwards sealed with a hydrogen/oxygen torch to create an approximately $25 \mathrm{~cm}$ long ampoule. $\mathrm{NiI}_{2}, \mathrm{CoI}_{2}$, and $\mathrm{FeI}_{2}$ were grown at temperatures of $700-730^{\circ} \mathrm{C}, 480-500^{\circ} \mathrm{C}, 480.500^{\circ} \mathrm{C}$ respectively for a period of about 150 hours. The higher (lower) figure indicates the temperature at the powder (growth) end of the ampoule. The resulting platelets were about $1 \mathrm{~cm}$ in diameter. 
Table 2.1 Ambient Pressure Properties of $\mathrm{NiI}_{2}, \mathrm{CoI}_{2}$, and $\mathrm{FeI}_{2}$

\begin{tabular}{|c|c|c|c|c|}
\hline Property & $\|$ & $\mathrm{NiI}_{2}$ & $\mathrm{CoI}_{2}$ & $\mathrm{FeI}_{2}$ \\
\hline Crystal Structure & $\|$ & $\begin{array}{l}\text { Hexagonal } \\
\left(\mathrm{CdCl}_{2}\right)\end{array}$ & $\begin{array}{l}\text { Hexagonal } \\
\left(\mathrm{CdI}_{2}\right)\end{array}$ & $\begin{array}{l}\text { Hexagonal } \\
\left(\mathrm{CdI}_{2}\right)\end{array}$ \\
\hline \multirow{2}{*}{$\begin{array}{l}\text { Lattice Constants } \\
\qquad \text { a, c ( } \AA)\end{array}$} & $\|$ & & & \\
\hline & $\|$ & $3.892,19.634$ & $3.96,6.65$ & $4.04,6.75$ \\
\hline Space Group & $\|$ & $D_{3 d}^{5}$ & $D_{3 d}^{3}$ & $D_{3 d}^{3}$ \\
\hline$T_{\mathrm{N}}$ (Kelvin) & $\|$ & 75 & $3-12$ & 10 \\
\hline $\mathbf{S}$ & $\|$ & 1 & $3 / 2$ & 2 \\
\hline \multirow{3}{*}{$\begin{array}{l}\text { Ground-state } \\
\text { representation of } 3 d^{\mathrm{h}} \\
\text { within the } D_{3 \mathrm{~d}} \text { point } \\
\text { group }\end{array}$} & $\|$ & & & \\
\hline & $\begin{array}{l}\| \\
\|\end{array}$ & ${ }^{3} A_{2 \mathrm{~B}}$ & ${ }^{4} T_{1 \mathrm{~g}}$ & ${ }^{s} E_{8}$ \\
\hline & $\|$ & & & \\
\hline \multirow[t]{3}{*}{$\Delta(e V)$} & $\|$ & 1.5 [ref. 9] & 4 [ref. 23] & 4 [ref. 23] \\
\hline & $\|$ & 4-5 [ref. 23] & & \\
\hline & $\|$ & & & \\
\hline$U(\mathrm{eV})$ & $\|$ & 4.5 [ref. 9] & n.a. & n.a. \\
\hline
\end{tabular}




\section{Figure Captions for Chapter II}

Fig. 2.1 Crystal structures of a) $\mathrm{CoI}_{2}$, and $\mathrm{FeI}_{2}$ and b) $\mathrm{NiI}_{2}$ [15]. The small black atoms are the transition-metal ions while the larger shaded ones are the iodide ions. The diagram to the left in figure a) shows the projection of the ions onto the $a b$ plane. The lattice constants appear in Table 2.1.

Fig. 2.2 Schematic density of states of the transition-metal iodides at atmospheric pressure. The upper and lower Hubbard bands are comprised of the transition-metal $3 d$ states and separated by the onsite Coulomb repulsion $U$. The charge-transfer gap ( $\left.E_{\text {CTG }}\right)$ is the gap between the iodine $5 p$ valence band and the upper Hubbard band. The transition-metal iodides are thought to be charge-transfer insulators.

Fig. 2.3 Band-structure calculation of $\mathrm{NiBr}_{2}$ [21] showing the transition-metal $4 s$ and $3 d$ bands and anion $p$ bands. The Fermi level lies within the $3 d$ bands.

Fig. 2.4 Optical and Near-IR absorption spectra of $\mathrm{NiCl}_{2}$ and $\mathrm{NiBr}_{2}$ taken at temperatures of (A) $300 \mathrm{~K}$ and (B) $5 \mathrm{~K}$ from ref. [25]. The intra-d transitions are marked by their symmetry assignments. Notice that the absorption edge shifts toward lower energies as one moves from $\mathrm{NiCl}_{2}$ to $\mathrm{NiBr}_{2}$ while the positions of intra-d transitions is rather constant. 

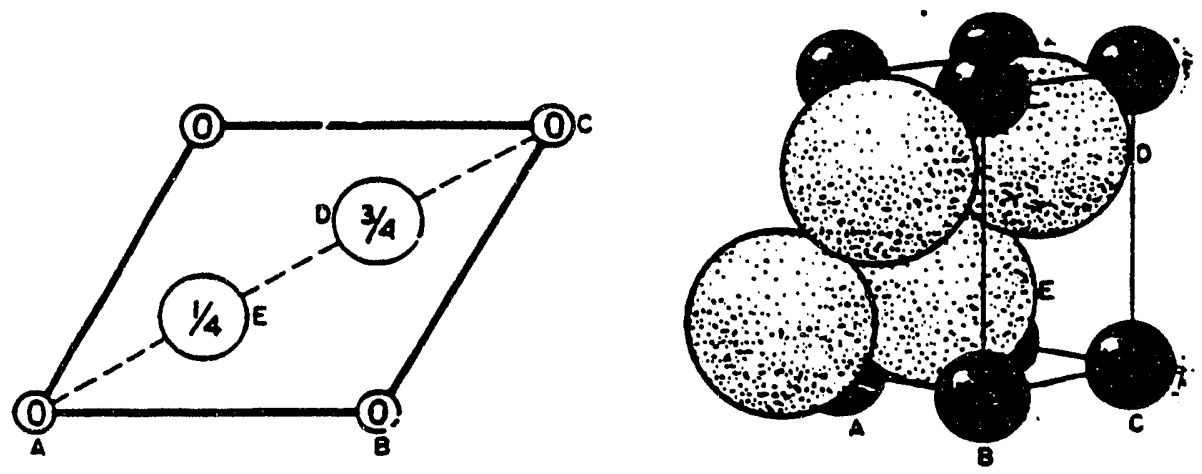

a)

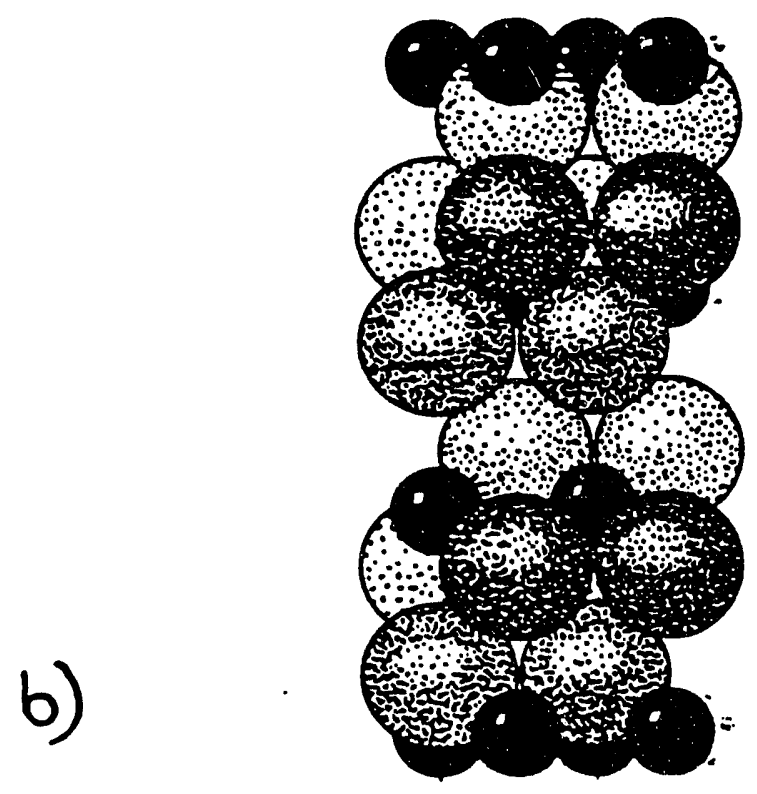

Fig. 2.1 


\section{Pressure $=0$}

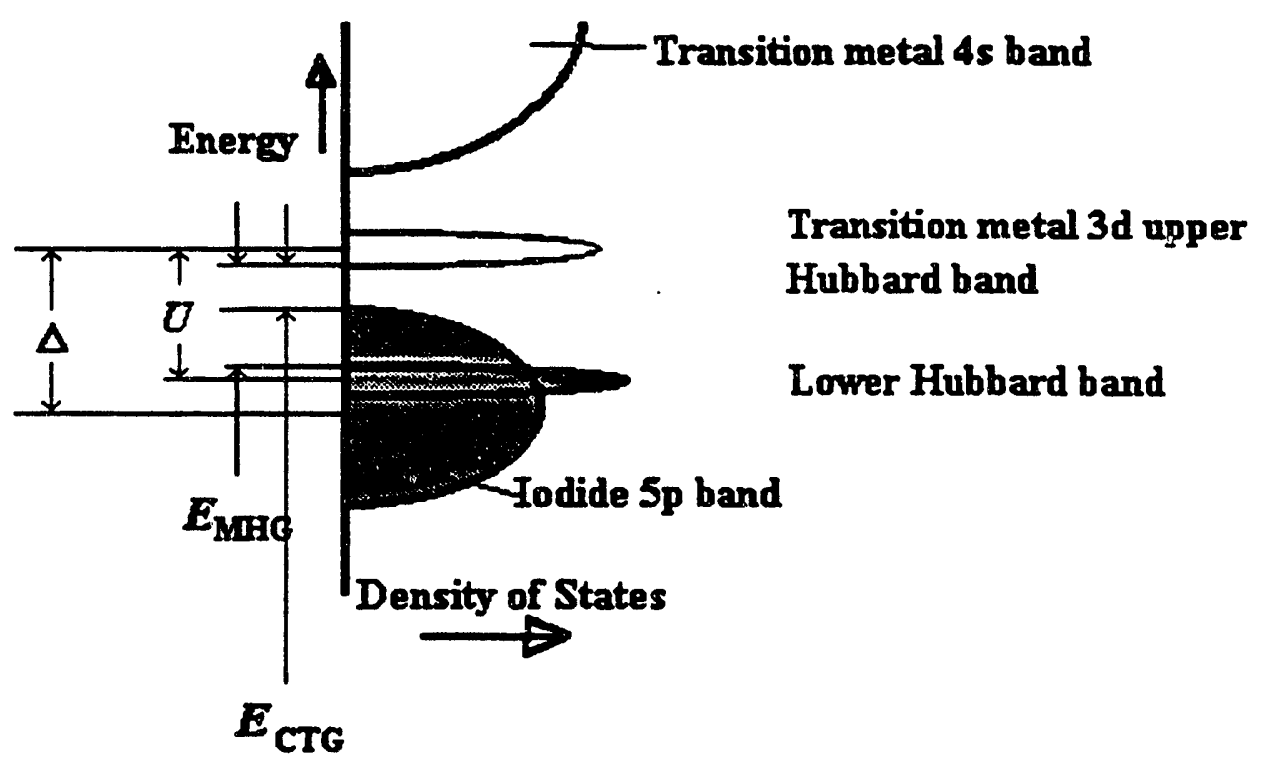

Fig. 2.2 


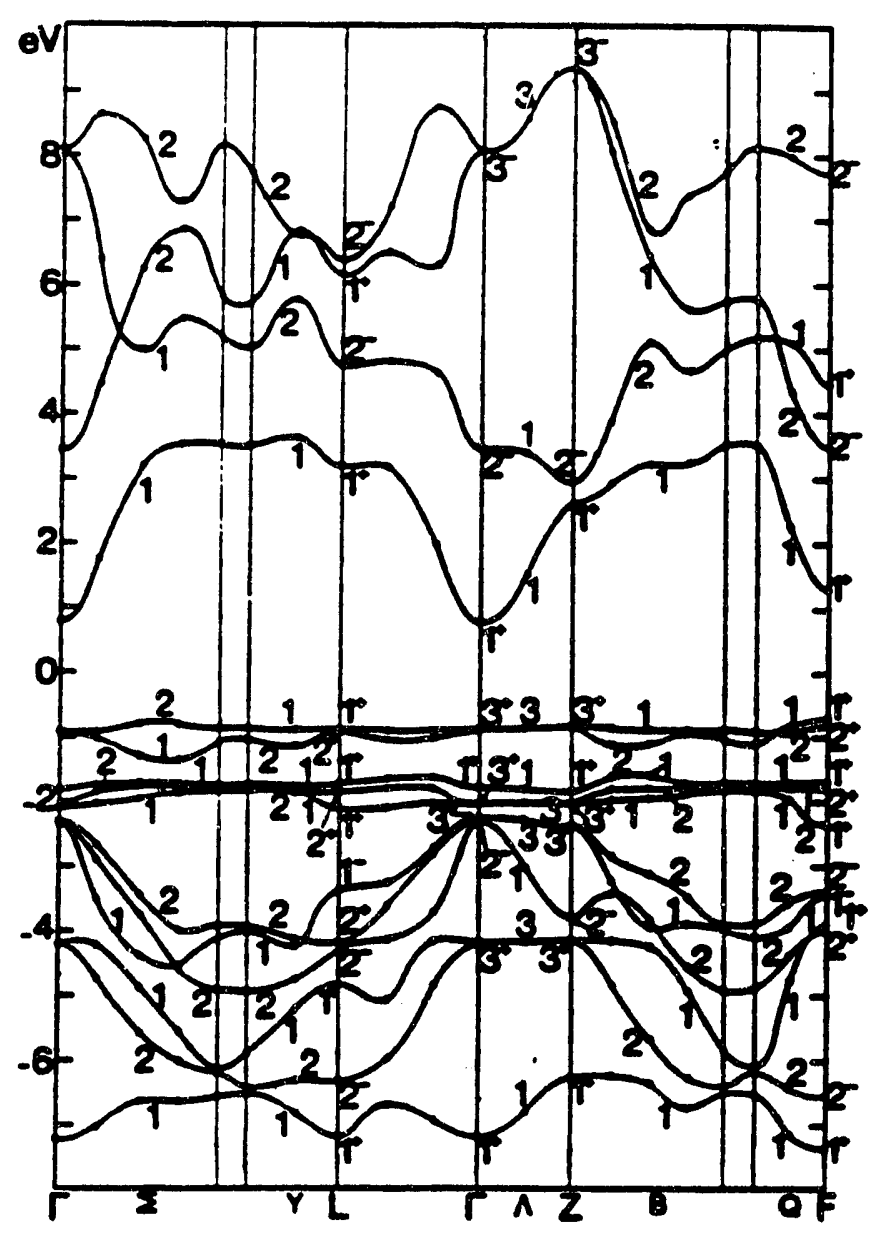

Fig. 2.3 

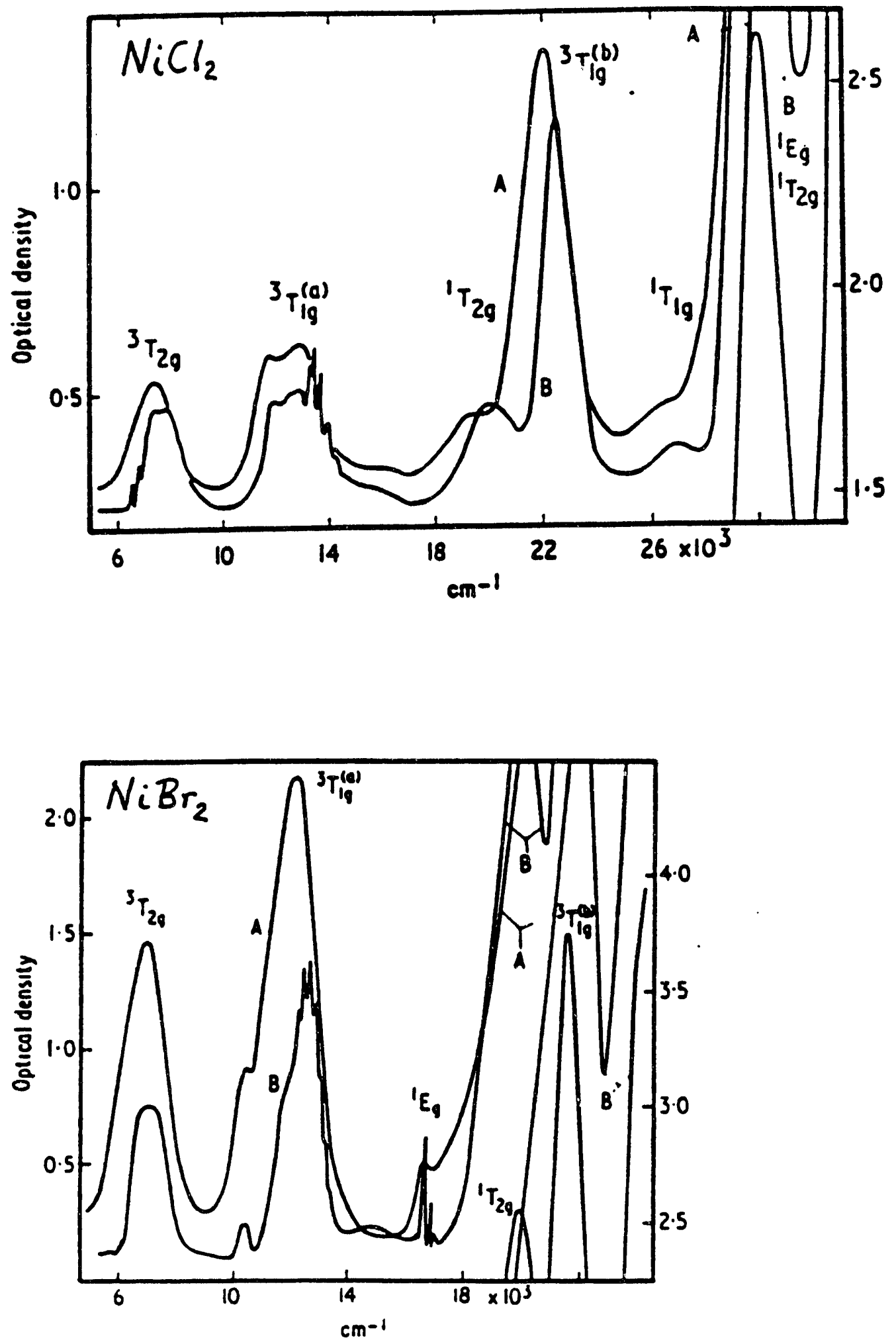

Fig. 2.4 


\section{Chapter III: Theoretical Considerations of Band-Overlap}

\section{Insulator-Metal Transitions and Magnetic Transitions}

\section{A: Insulator-Metal Transitions and Band Overlap}

An insulator is a solid that has an energy gap, $E_{\mathrm{g}}$ separating a filled valence band and an empty conduction band. An IM transition occurs when the valence and conduction bands overlap. The band movement may be induced by changing an external variable such as hydrostatic stress and temperature or by varying the material composition by alloying.

If one considers the simplest case of band overlap involving non-interacting electrons, the number of conduction electrons increases continuously as $E_{\mathrm{g}}$ decreases and the $\mathrm{IM}$ transition is second-order at zero temperature $[2,26]$. There is no phase transition at finite temperature. We can visualize this in the following way. In an ideal, perfect insulator the number of free carriers at a finite temperature $T$ is limited to a small number of charges that are thermally excited across the energy gap. As $E_{\mathrm{g}}$ decreases to the point where $E_{\mathrm{g}} \sim k T$, the number of free carriers becomes significantly higher. (See Fig. 3.1) Once the bands overlap the solio becomes a metal, but there is no significant change in the number of conduction electrons when this occurs. As the bands continue to overlap, the number of carriers increases continuously as electrons spill over from the valence band to the conduction band. The number of conduction electrons always increases continuously regardless of the temperature. Its derivative with respect to $E_{\mathrm{B}}$ will also be continuous except at $T=0$. At $T=0$ there will be a kink in the carrier concentration as a function of $E_{\mathrm{g}}$. Since the carrier concentration itself is continuous but its derivative with respect to $E_{\mathrm{g}}$ is not, the transition at $T=0$ is second-order.

We can make a simple model calculation of the number of conduction electrons, $n$, as a function of the energy gap $\left(E_{\mathrm{g}}\right)$. The expression for $n$ is 


$$
\begin{aligned}
& n=\int_{E_{\mathrm{F}}}^{\infty} N(E) f(E) d E \\
& f(E) \equiv \frac{1}{\exp \left(\left(E-E_{\mathrm{F}}\right) / k T\right)+1}
\end{aligned}
$$

where $f(E)$ is the Fermi factor and $N(E)$ is the density of states. For three-dimensional parabolic valence and conduction bands with equal effective masses, $N(E)$ is

$$
\begin{gathered}
N(E)=\left\{\begin{array}{l}
\alpha\left(\sqrt{E-E_{\mathrm{g}} / 2}\right) \theta\left(E-E_{\mathrm{g}} / 2\right) \\
+\alpha\left(\sqrt{-E-E_{\mathrm{g}} / 2}\right) \theta\left(-E-E_{\mathrm{g}} / 2\right)
\end{array}\right\} \\
\text { where } \theta(x) \equiv\left\{\begin{array}{ll}
1, & x \geq 0, \\
0, & x<0
\end{array}\right\} .
\end{gathered}
$$

$\theta(x)$ is a step function. The Fermi level, $E_{\mathrm{F}}$, will remain half way between the top of the valence band and the bottom of the conduction band even as the bands continue to overlap. In the insulating regime $\left(E_{\mathrm{g}}>>h T\right), f(E)$ can be approximated by a Boltzmann distribution function and the integral evaluated in closed form. The result is that $n$ varies as $\exp \left(-E_{\mathrm{g}} / 2 k T\right)$. On the other hand, when $E_{\mathrm{g}} \ll<-k T$ (metallic phase), most of the conduction electrons exist within an energy $k T$ of the Fermi surface and $n \propto N\left(E_{\mathrm{F}}\right) k T \propto\left(\sqrt{\left|E_{\mathrm{g}}\right| / 2}\right) k T$. The Fig. 3.2 shows the number of conduction electrons as a function of the energy gap at finite temperature. Notice that the electron concentration varies smoothly. Examples of band-overlap transitions include the metals $\mathrm{Yb}$ and $\mathrm{Ba}$. The application of pressure induces a metal-to-insulator transition in these materials by separating the valence and conduction bands [27]. The metallizations of both $\mathrm{Xe}$ and $\mathrm{H}_{2}$ under pressure are also believed to be caused by band overlap $[13,14]$.

Physically the number of conduction electrons is reflected in the resistivity of the sample via the expression 


$$
\rho=\frac{1}{\left(n \mu_{e}+p \mu_{h}\right) e},
$$

where $\mu_{\mathrm{e}}$ and $\mu_{\mathrm{h}}$ are the mobilities of the electrons and holes respectively and $e$ is the electron charge. If the mobilities are assumed to remain equal and constant as the bands overlap, the resistivity at constant temperature behaves as $1 / n$. In Fig. 3.2 we have also plotted $\log (1 / n)$ vs. $E_{g} k T$ to show the qualitative behavior of the resistivity during a bandoverlap metallization. Notice that in this simple model, $\log (\rho)$ decreases linearly with $E_{\mathrm{g}}$ until $E_{\mathrm{g}}$ vanishes. After metallization the resistivity becomes comparatively independent of $E_{\mathbf{g}}$

If we include electron-electron interaction, the situation becomes more interesting. The lowest lying optical excitations across the energy gap require an energy less than $E_{\mathrm{B}}$ because the electron and hole attract each other to form an exciton.

$$
\hbar \omega=E_{\mathrm{g}}-E_{\mathrm{ex}} .
$$

$E_{\text {ex }}$ is the exciton binding energy given crudely by

$$
E_{\text {ex }} \sim 13.6\left(\frac{\mu_{r}^{2}}{\varepsilon}\right) \mathrm{eV},
$$

where $\mu_{\mathrm{r}}$ is the reduced mass defined by $1 / \mu_{\mathrm{r}}=1 / m_{\mathrm{e}}+1 / m_{\mathrm{h}}$ and $\varepsilon$ is the dielectric constant. When $E_{g}$ is less than or equal to the exciton binding energy, excitons will form spontaneously $[2,26]$ causing a first-order transition. $E_{\text {ex }}$ is expected to diminish with decreasing $E_{\mathrm{g}}$ because the dielectric function $\varepsilon$ will increase. Furthermore, an attractive exciton-exciton interaction causes a high density of excitons to form a state of condensed electrons and holes with an additional cohesive energy. There is a critical density where the cohesive energy per exciton, $E_{\mathrm{c}}$, is maximum and excitons condense into an electronhole liquid. The formation of an electron-hole liquid was thoroughly studied in germanium where the critical density is $-10^{17} \mathrm{~cm}^{-3}$ [28]. Thus a first-order transition into this electron-hole liquid will happen when 


$$
E_{\mathrm{g}} \sim E_{\mathrm{ex}}+E_{\mathrm{c}}
$$

Although free excitons cannot contribute to the conductivity because they are neutral, in the liquid phase the electrons and holes form a plasma that can have metallic properties.

\section{B: Magnetic Transitions}

In many rare-earth elements and their compounds, antiferromagnetic (or ferromagnetic) to diamagnetic transitions have been observed. These have been accompanied by first-order IM transitions (e.g. Ce metal, SmTe, EuO [29]). These transitions have been modeled by simple Hamiltonians that assume the crossing of a nonmagnetic band with an exchange-split Hubbard band that carries magnetic moments. Metallization is caused by band overlap. Disappearance of the magnetic moments is caused by filling of the magnetic band. In these models only the relative positions of the bands and the position of the Fermi level changes as a function of temperature or pressure. The character of the electronic wavefunctions are assumed to remain intact across the transition. An excitonic interaction between the bands is included. Such models have been successful in qualitatively describing the transitions.

After it was discovered that the magnetic transition (to be described in Chapter $\mathrm{V}$ ) in $\mathrm{NiI}_{2}$ was accompanied by an $\mathrm{MM}$ transition (discussed in Chapter VI), Giesekus et. al. [30] and Freericks et. al. [31] nuticed the similarity between this transition and those in the rare-earth compounds. They performed similar model calculations using the following Hamiltonian [31].

$$
\begin{gathered}
H=\sum_{v * \sigma} \varepsilon_{v}(\mathrm{k}) a^{*}{ }_{v * \sigma} a_{v * \sigma}+\varepsilon_{d} \sum_{i \sigma} d^{*}{ }_{i \sigma} d_{i \sigma}-G \sum_{v \sigma} c^{*}{ }_{v \sigma} c_{v \sigma} d^{*}{ }_{i \sigma} d_{i \sigma} \\
+\sum_{i j} J_{i j}\left(1-d^{*}{ }_{i \uparrow} d_{i \uparrow}-d^{*}{ }_{i \downarrow} d_{i \downarrow}\right) \mathbf{S}_{i} \bullet \mathbf{S}_{j}\left(1-d^{*}{ }_{j \uparrow} d_{j \uparrow}-d^{*}{ }_{j \downarrow} d_{j \downarrow}\right) \\
\text { where } c^{*}{ }_{v \sigma \sigma} \equiv \frac{1}{\sqrt{N}} \sum_{k} \exp \left(-k \bullet \mathbf{R}_{\mathrm{i}}\right) a^{*}{ }_{v * \sigma}
\end{gathered}
$$


The terms of the Hamiltonian are summarized below.

i) $a^{*}{ }_{w 0}$ creates Bloch holes in filled anion $p$ band $v$ with wavevector $k$ and spin $\sigma$. The energy of a hole in band $v$ is denoted by $\varepsilon_{v}(k)$.

ii) $\varepsilon_{d}$ is the energy of a dispersionless $d$ orbital localized on site $i$ with spin $\sigma$ created with operator $d_{\text {io }}^{*}$. By definition, this band is separated from the $p$ band by the charge-transfer energy $\Delta$.

iii) $G$ is an interaction potential between a hole in the $p$ band and an electron in the $d$ band both localized at the site and with spin $\sigma$. The creation operator of a Wannier hole orbital from band $v$ localized about site $i$ with spin $\sigma$ is cienoted by $c^{*}{ }_{\text {no }}$.

iv) $J_{i j}$ is the exchange parameter between $d$ electrons on sites $i$ and $j$.

The only effect of the applied pressure is assımed to be a linear decrease in $\Delta$. Using a mean-field approximation, the model was solved by Freericks and Falicov for various values of the parameters $G, J$, and $\Delta[31]$. They calculated the phases at non-zero temperature and found a first-order phase line separating an antiferromagnetic insulating state from a diamagnetic metallic state with a cri:cal point at about $1500 \mathrm{~K}$ and the usual second-order antiferromagnetic-paramagnetic phase line (Fig. 3.3). This calculation predicts that metallization and disappearance of magnetic moments happen simultaneously before $\Delta$ vanishes. On the other hand Giesekus and Falicov [30] performed a calculation at $T=0$ including $p-d$ hybridization. They obtained a rich phase diagram (Fig. 3.4) that for different values of $G$, the interaction strength, predicts a first-order $I M$ transition only, two second-order transitions, or both first and second-order phase transitions depending on the strength of $G$. If both first and second-order transitions occur, one of the intermediate phases will be a weakly antiferromagnetic metallic phase. The ratio $\mathrm{E} / \mathrm{W}$ (the abscissa in Fig. 3.4) is essentially a normalized $\Delta$ while the ordinate $G / W$ is the normalized interaction strength. 
Although these model calculations and theoretical considerations are not quantitative, they do provide insight into the physics of these transitions, namely a physical reason for a connection between the magnetic and $\mathrm{IM}$ transitions and an explanation for the possibility of observing both first and second-order phase transitions. 


\section{Figure Captions for Chapter III}

Fig. 3.1 A band-overlap insulator-metal transition. a) Insulating phase. b) Metallic phase.

Fig. 3.2 Number of conduction electrons as a function of the normalized energy gap during a band-overlap $\mathrm{IM}$ transition. The calculation assumes a valence and a conduction band of equal parabolicity. The transition from an insulating phase $\left(E_{\mathrm{B}}>0\right)$ to a metallic phase $\left(E_{\mathrm{g}}<0\right)$ is a second-order phase transition at zero temperature. There is no phase transition at finite temperatures.

Fig. 3.3 Calculated phase diagram of $\mathrm{NiI}_{2}$ from Ref. [31]. The chained line is a secondorder phase line separating the antiferromagnetic phase (below) from the paramagnetic phase (above). The solid line represents a first-order insulator (left) to metal (right) transition. This phase line ends at high temperatures with a classical critical point. The dotted line represents where the number of solutions to their model Hamiltonian changes. Note that at room temperature the $\mathrm{IM}$ trarsition happens before $\Delta$ goes to zero.

Fig. 3.4 Calculated zero-temperature phase diagram of $\mathrm{NiI}_{2}$ from Ref. [30] based on a model which includes $p-d$ hybridization. $G / W$ (the $y$-axis) is the interaction energy between $p$ and $d$ electrons normalized to the $p$ band width $\mathrm{W}$. The $\mathrm{x}$-axis $(\mathrm{E} / \mathrm{W})$ is the normalized, average energy difference between the $d$ and $p$ electrons. $m=1$ labels the region where the magnetic moments are at their me imum possible value. $m=0$ labels the non-magnetic region. In between there are several possibilities depending on the value of $G / W$. The thick line indicates a first-order transition (discontinuous change in $m$ ) while the thin lines mark second-order transitions. The first-order phase line ends in a classical critical point. 


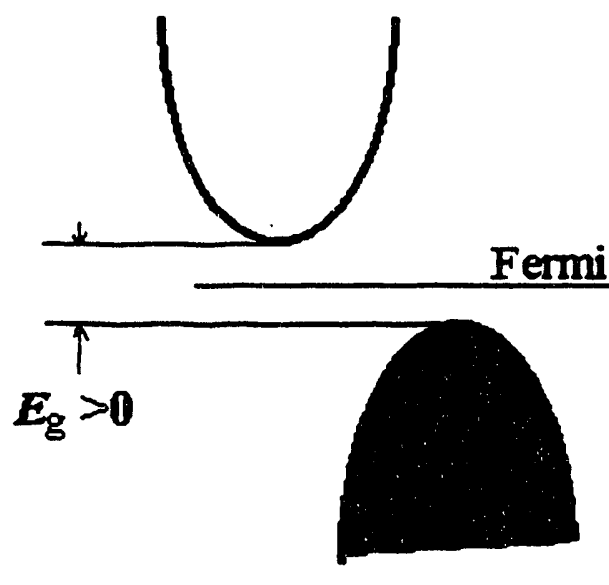

a) Insulator

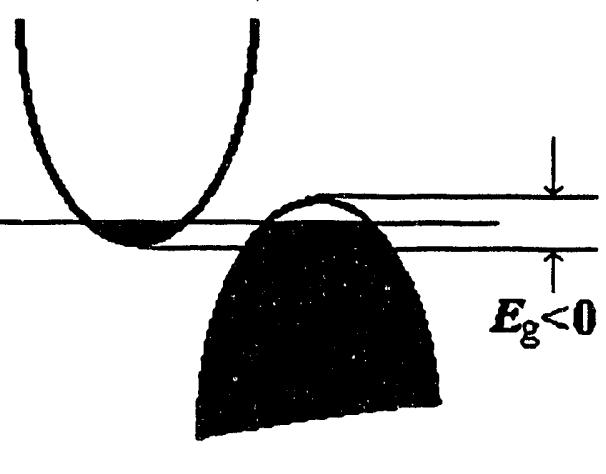

b) Metal

Fig. 3.1 


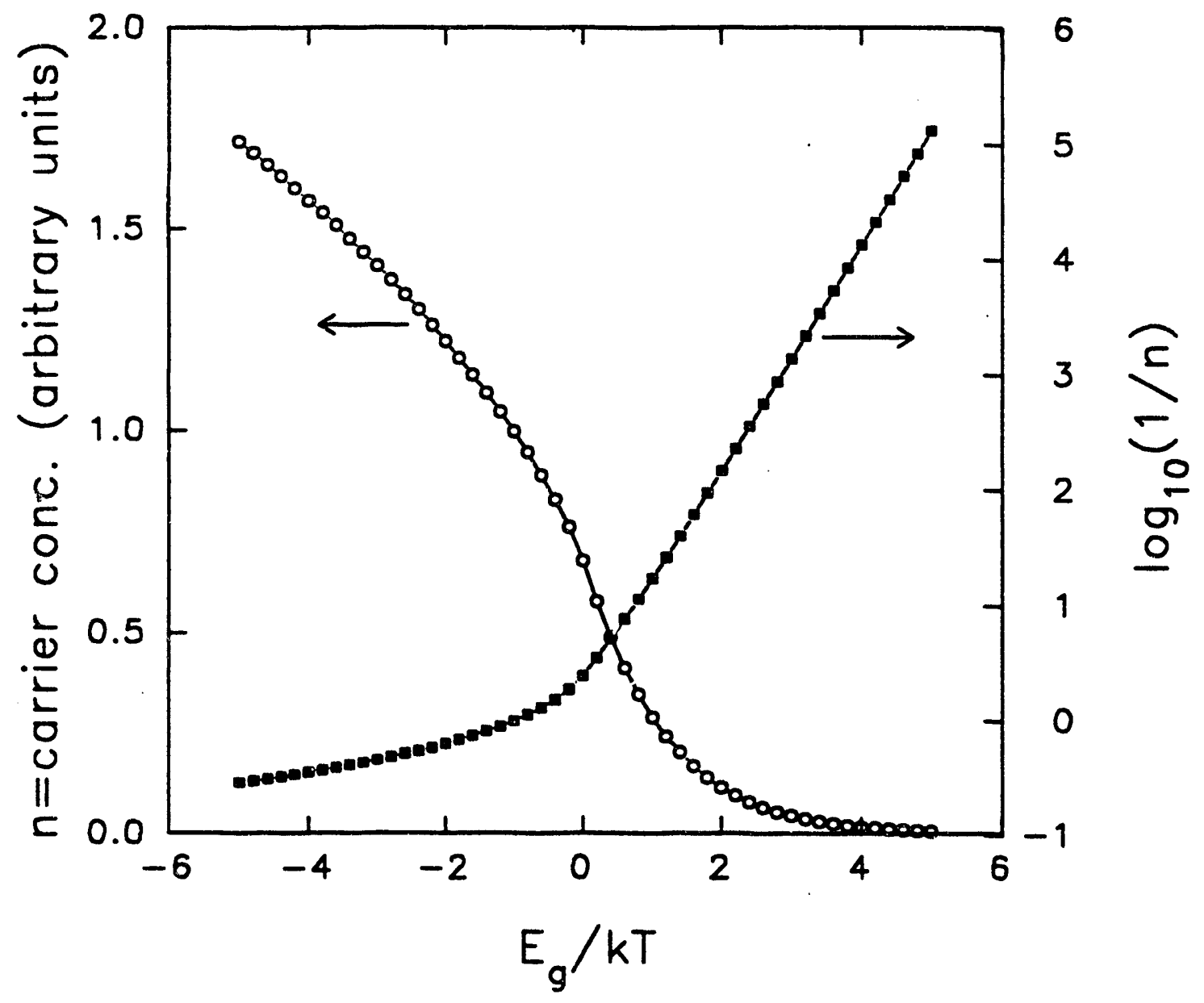

Fig. 3.2 


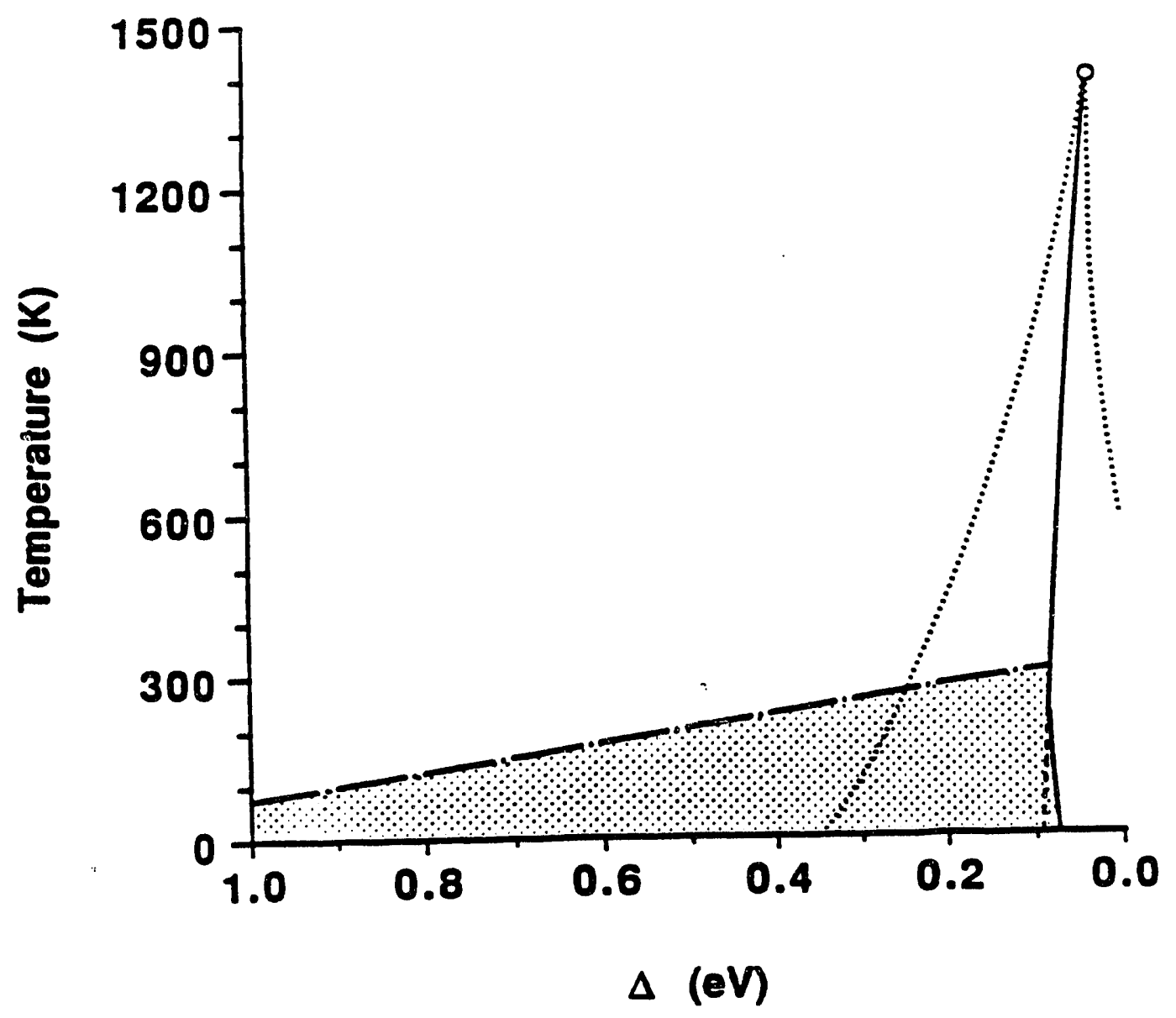

Fig. 3.3 


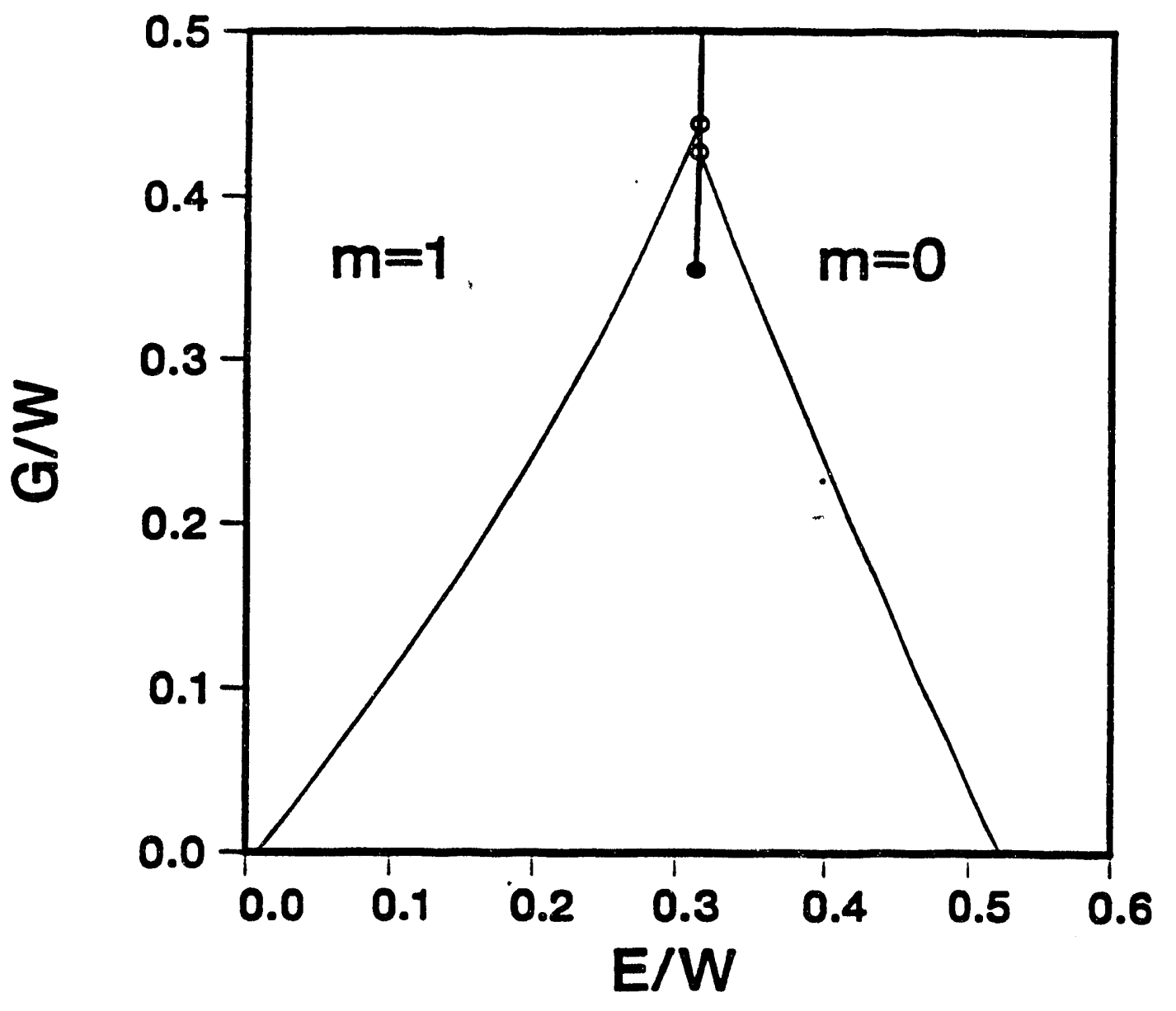

Fig. 3.4 


\section{Chapter IV: Measurements Under High Pressure:}

\section{Experimental Procedure}

We performed both resistivity and absorption measurements at high pressure. The high pressures were achieved with a piston-cylinder-type diamond-anvil cell (DAC). Since the DAC is has been used extensively for more than a decade and extensive review articles exist describing their design and function, we refer the reader to a thorough review article for details of its construction and use [32].

For reference, the components of the DAC that we used are illustrated and labeled in Fig. 4.1. The pressure was initially applied by an external hydraulic press and maintained by tightening the lock ring collar. This type of cell is extremely compact and can be easily fitted into an optical cryostat for low temperature measurements. However, tightening the lock ring invariably increased the pressure in the cell making it difficult to obtain precisely a desired pressure. When precise pressure control was necessary, we used a vise-like device called a "nutcracker" which enabled us to increase the pressure gradually in small increments by tightening a nut. For pressure calibration we used the shift of the $\mathrm{R} 1$ fluorescence line of nuby with a conversion factor of $0.274 \mathrm{GPa} / \mathrm{A}$.

The procedures for making resistivity and absorption measurements are described below.

\section{A: Resistivity Measurements Under High Pressure}

Performing resistivity measurements inside a DAC is difficult because the wires must be insulated from the metal gasket. We accomplished this by placing powdered $\mathrm{Al}_{2} \mathrm{O}_{3}$ powder on the gasket and precompressing it to form a disk of compacted insulating powder. Because $\mathrm{Al}_{2} \mathrm{O}_{3}$ is very hard, it is not a suitable pressure medium. Furthermore the use of powdered $\mathrm{Al}_{2} \mathrm{O}_{3}$ as an insulating layer precludes the use of a liquid as the pressure medium. Thus in the gasket hole, $\mathrm{CaSO}_{4}$ powder, which is considerably softer, is 
used instead. This two-powder method was developed by D. Erskine et. al. [33]. The details of our technique are described in the next paragraph.

We assume that one is starting with a cell outfitted with diamond anvils that have been aligned parallel to each other within one optical fringe. The first step is to make an electrical feedthrough from the inside of the DAC to the outside. We passed six insulated, $0.5 \mathrm{~mm}$ diameter copper wires through a hole located on the upper half of the DAC and attached them to the metal support holding the diamond with either epoxy or stycast 2850FT. Four of the wires were used to measure the sample resistivity; the other two were connected to a silicon diode thermometer mounted adjacent to the diamond. The stripped ends of the wires inside the cell were turned upward to serve as mounting posts. A Teflon sheath was placed over the wires from the outside to protect the wires from external damage. The outside ends of the wires were soldered to a connector.

Samples are mounted using this procedure. A gasket of appropriate metal is chosen, mounted on the bottom half of the DAC with duct seal, and preindented to the highest pressures one would like to achieve during the experiment. The gasket is removed and a hole about 1/3 the diameter of the culet is drilled in its center. Any metal burrs on the back side of the gasket resulting from the drilling procedure are removed. After cleaning the culets with a cotton swab, the gasket is replaced on the anvil in the same position as when it was preindented. This assures alignment of the culet corners. The gasket should be snugly held by the duct seal; it is important that it does not move from this point on. A small wire in attached from the gasket to the body of the DAC with conducting silver paint assuring electrical grounding of the gasket and enabling one to detect undesirable electrical shorts of the measurement wires to the gasket. The upper surface of the gasket surrounding the indentation area is then painted with epoxy to prevent accidental shorting of wires from the upper half of the DAC. It is crucial that the epoxy partly coats the sides of the indentation crater but not the flat area where the culet of the upper diamond will eventually press on the gasket. The epoxy in this region helps 
to secure the $\mathrm{Al}_{2} \mathrm{O}_{3}$ powder. After the epoxy has hardened, a layer of $0.5 \mu \mathrm{m} \mathrm{Al}_{2} \mathrm{O}_{3}$ powder is compacted into the indentation area. The gasket hole area should be cleaned of $\mathrm{Al}_{2} \mathrm{O}_{3}$ powder and then filled with $\mathrm{CaSO}_{4}$ powder. The DAC is carefully assembled and the pressed by hand to compact both powders. When the DAC is reopened, the powder should not stick to the upper diamond. The surface of the compacted powder should be flat and seem slightly translucent. One should be able to distinguish the two powders. This process is repeated until three layers of the $\mathrm{CaSO}_{4}$ and $\mathrm{Al}_{2} \mathrm{O}_{3}$ powder base are laid down and compacted. The completed arrangement is schematically shown in Fig. 4.2. The lower half of the DAC is then set aside while we place wires on the upper half.

We used $12.5 \mu \mathrm{m}$ diameter $(0.5 \mathrm{mil})$ gold wires to make electrical contact from the copper wire posts to the sample. Gold is best because it is highly ductile. One of the chief reasons for failure of this technique is that the edge of the culet has a tendency to sever the wires. The other main reason for failure is the shorting of the gold wires through the $\mathrm{Al}_{2} \mathrm{O}_{3}$ powder at high pressures. The latter problem can be diminished by using thinner gold wires. Because thin wires are difficult to handle, thinner wires are only used in the vicinity of the culet where shorting most likely occurs. The wire can be made thinner by rolling it into a thin sheet with a steel ball bearing. One can then cut the resulting sheet into triangular wedges about 200-300 microns in length with a surgical knife with the help of a stereo-microscope. The wedge shaped leaves of gold are then glued to the culet of the upper diamond using tiny dabs of epoxy. These are arranged in a star fashion pointing toward the center (see Fig. 4.3). Thicker $0.5 \mathrm{mil}$ wires are then cut to the proper length and then glued to the side of the diamond so as to make physical contact with the wedge shaped leaves. When the cell is eventually closed the pressure will bond the 0.5 mil wire to the thin leaves of gold. When the glue has dried, the other ends of the 0.5 mil wires are wrapped around the copper-wire posts and electrically attached with a small amount of conducting silver paint. Several small ruby chips are placed in the center of the star pattern of gold wedges for measuring the pressure by the ruby fluorescence method. 
The transition-metal halides are quite hygroscopic. They must be loaded into the DAC under a dry atmosphere inside a glove box or similar bag. The operator should wear latex gloves. We used nitrogen gas to purge the glove box of moisture. Because dry atmospheres are conducive to the build up of static electricity, one should carefully ground all instruments used in manipulating the small samples. Otherwise one may find that as one tries to touch a small sample it will be repelled and often disappears. Furthermore, the compressed powder invariably holds some moisture. We placed the bottom half of the cell in a desiccator for about 12 hours to drive off any residual moisture. Even with this precaution the $\mathrm{FeI}_{2}$ samples were sometimes visibly altered after loading into the DAC for resistivity measurements.

The samples were cut in a dry environment to dimensions of about $50 \times 50 \times 10 \mu \mathrm{m}^{3}$ and placed on the $\mathrm{CaSO}_{4}$ powder at the center of the culet indentation on the lower half of the DAC. A powder pressure medium inherently introduces some pressure variation across the surface of the culet. Across the culet we typically encountered pressure gradients of \pm 8 percent for pressures below $20 \mathrm{GPa}$. The use of smaller samples is one way to minimize the pressure variation across the sample. The DAC was then carefully assembled and the diamond anvils brought together. As the DAC was closed, the goldleaf wires on the upper half of the cell made physical contact with the sample. No special treatment was made to insure the ohmic character of this contact.

The resistance measurement technique reported in ref. [33] is referred to as a quasi-four probe technique. In that method two loops of wire were used to contact the sample in a way that four leads to the sample are formed. There were, however, only two contacts. The current was passed along two leads while the voltage was measured along the other two. Although this method does avoid measurement of the resistance of the wires, it does not avoid measurement of the contact resistance. We improved this technique by making a true four-probe measurement that uses four wires and four contact points. We were able to measure the contact resistance and the sample resistance and 
found that, for our samples, the value of the contact resistance under pressure followed the value of the sample resistance, but always remained about 10 times higher. In other words, the general behavior of the contact resistance followed the sample resistance so that interpretations of $\mathrm{IM}$ transitions based on the quasi-four probe measurements remained valid. However measuring more subtle effects require the use of a four-point technique. For example in our work on the superconductivity of As under pressure [34], a quasi-four probe technique would have sufficed for the measurement of the superconducting transition temperature because the proximity effect for superconductors insures that the contacts will superconduct when the sample does. But in determining the normal-state resistivity, a true four-probe technique was necessary.

Four-probe resistance measurements were made with d.c. currents ranging from 1 $\mu \mathrm{A}$ to $10 \mathrm{~mA}$. We estimated the resistivity of our samples from the resistance and the approximate dimensions given above. Two kinds of resistivity measurements were performed. In the first the sample pressure was increased at room temperature in increments of $1 \mathrm{GPa}$ or less while the resistance was monitored as a function of pressure. In the second the sample pressure was set at room temperature and the resistance was measured as a function of temperature as the cell was cooled to liquid-helium temperatures. The silicon diode thermometer was biased at $10 \mu \mathrm{A}$ of current. Because of thermal contraction, the pressure inside the cell typically increased by about $10 \%$ as the cell was cooled from room temperature down to $77 \mathrm{~K}$.

\section{B: Near-IR Absorption Measurements Under High Pressure}

We utilize the transparency of diamond to photons of energies between 0.3 and 5 $\mathrm{eV}$ to perform absorption measurements under pressure. Specifically, absorption measurements can be used to measure the energy of the band gap under pressure and determine by extrapolation the metallization pressure. The measurements can be extended farther into the infrared if one uses type $\Pi$ a diamonds which are free from nitrogen 
aggregates. Even though absorption measurements can only be performed above $0.3 \mathrm{eV}$ with the more common type I diamond, they are an excellent complement to the resistivity measurements which are more sensitive to smaller activation energies.

Loading the samples into the DAC for absorption measurements is comparatively easy. We preindented the gaskets as described above and drilled gasket holes of about $200 \mu \mathrm{m}$ in diameter. Again several ruby chips were placed into the gasket hole to serve as pressure indicators. As before, the samples were loaded under dry nitrogen gas inside a glove box. The transition-metal iodides are weakly bonded along the $\mathrm{c}$-axis and can be easily cleaved into thin sheets that are ideal for absorption measurements. To minimize light leakage around the sample during absorption measurements, we usually covered the entire gasket hole with one thin sample sheet .

For the absorption experiments we used liquid nitrogen $\left(\mathrm{LN}_{2}\right)$ as the pressure medium because it is transparent, is much softer than powders under high pressure, and does not chemically affect our samples. To load the DAC with $\mathrm{LN}_{2}$ the cell must be immersed in the liquid and closed only after its temperature has equilibrated with the surrounding liquid. The procedure is as follows. After the sample is in place, we close the cell, screw on the lock ring until it is finger tight, and then loosen it by $1 / 6$ of a turn. At this point the cell is slightly open. The DAC is then placed in the "nutcracker", a vise-like device that allows the pressure of the DAC to be increased by tightening a nut. The nut is tightened by hand and then loosened by $1 / 2$ turn. Afterwards the nutcracker with the DAC is immersed in a wide-mouth dewar filled with $\mathrm{LN}_{2}$. The entire nutcracker must be covered with the liquid. Once the bubbling stops, the nut is tightened by about one and half turns. The amount of tightening necessary to trap the liquid depends on the size of the diamond culets and the type of gasket used, and some experimenting is inevitably necessary. It is imperative to use the spring-like Bellevue washers with the nutcracker so that the pressure is applied gradually. Otherwise breakage of the diamonds may result. 
The absorption apparatus (shown in Fig. 4.4) consisted of a 250 Watt quartz tungsten-halogen lamp, a 1/4 meter Instruments, SA monochromater, a light chopper, and a $\mathrm{PbS}$ photodetector. Light emanating from the lamp was focused onto the entrance slit of the monochromater with a spherical mirror. The exiting, monochromatic light was chopped at $383 \mathrm{~Hz}$ and passed through an appropriate low-pass filter to eliminate higher harmonics of the fundamental light frequency. A reflecting microscope objective was used to focus the light into the DAC. After passing through the DAC, the light was collected by an $\mathbb{R}$ grade fused silica lens and focused onto the PbS detector biased by a $180 \mathrm{~V}$ battery in series with a $6 \mathrm{M} \Omega$ resistor. The signal was detected by a lock-in amplifier. Both the monochromater and the lock-in amplifier were interfaced to a computer via a IEEE-488 bus to automate the data-collection procedure. For wavelengths between 600 $1200 \mathrm{~nm}$ we used a 1200 line/mm grating. For wavelengths between $1000-2500 \mathrm{~nm}$, a 300 line/mm grating was used instead. The low-pass filters and their usable ranges are listed below.

$\begin{array}{ll}\text { Schott Glass Orange Filter No. OG570 } & 600-1000 \mathrm{~nm} \\ \text { IR transmitting filter for a FJW IR viewer } & 1000-1500 \mathrm{~nm} \\ \text { Silicon } & 1500-1800 \mathrm{~nm} \\ \text { Germanium } & 1800-2500 \mathrm{~nm}\end{array}$

All the absorption measurements were performed at room temperature.

To determine the absorption, we measured the transmittance of the DAC with $\left(T_{\text {somple }}\right)$, and without $\left(T_{\text {cal }}\right)$ the sample. From these transmittances, we calculated the absorbance, $\alpha x$, where $\alpha$ is the absorption coefficient and $x$ the sample thickness:

$$
\infty x=-\ln \left(\frac{T_{\text {sumple }}}{T_{\text {cal }}}\right)
$$


No attempt was made to correct for the reflectivity of the sample. Although this limits the accuracy of any quantitative interpretation, especially in the determination of line shapes, it cannot greatly alter our conclusions when prominent features such as a strong absorption edge or the position of a sharp peak are concerned. When $c x$ is below 1, reflectivity is an important issue. Since at ambient pressures the reflectivities of the TMIs are relatively constant below the absorption edge, it should not add any strong features to our absorption spectra but will more likely contribute a constant background. The $\alpha x$ measurable with our setup was limited by a detector noise of about $1 \mu \mathrm{V} / \sqrt{\mathrm{Hz}}$, which could be reduced by cooling the detector and using better shielding, and by stray light leakage around the sample. The latter was often the dominant factor. Typically we were able to measure $\infty x \leq 6$.

The lower limit of the photon energies accessible with our equipment was actually limited to $0.40 \mathrm{eV}$ by the transparency of the quartz used in the lamp's construction. For one sample, $\mathrm{NiI}_{2}$, we measured the absorption farther into the infrared (down to $0.3 \mathrm{eV}$ ) using a Digilab Fourier-transform infrared spectrometer. In this case, the samples were mounted in a Merrill-Bassett type DAC [35] and the measurements were performed at licuid-He temperatures. For details of the apparatus and experimental procedure we refer the reader to the existing literature [36]. 


\section{Figure Captions for Chapter IV}

Fig. 4.1 Schematic drawing of a piston-cylinder type DAC. The diamonds are supported by tungsten-carbide disks. The culets are made parallel by adjusting a hemispherical rocker on the jower half and aligned laterally by a set screw on the upper half. The pressure is applied externally by a hydraulic press and then maintained by tightening the lock ring.

Fig. 4.2 Gasket preparation for resistivity measurements. A preindented metal gasket is drilled. Compacted layers of epoxy glue and aluminum-oxide powder are used to insulate the wires from the metal gasket. Soft calcium-sulfate powder is used to ensure a quasihydrostatic environment in the gasket hole.

Fig. 4.3 Wiring of the DAC for resistivity measurements. Wedge shaped gold leaflets are glued to the diamond anvil and connected to $0.5 \mathrm{mil} \mathrm{Au}$ wire held down by epoxy glue. The $0.5 \mathrm{mil}$ Au wires are electrically connected to the larger wires by silver paint.

Fig. 4.4 Experimental setup for absorption measurements. Light from the 250 Watt quartz halogen lamp was focused into the DAC with mirror optics to minimize chromatic aberration. The light was chopped and detected by a $\mathrm{PbS}$ photoconductor with a lock-in amplifier. 


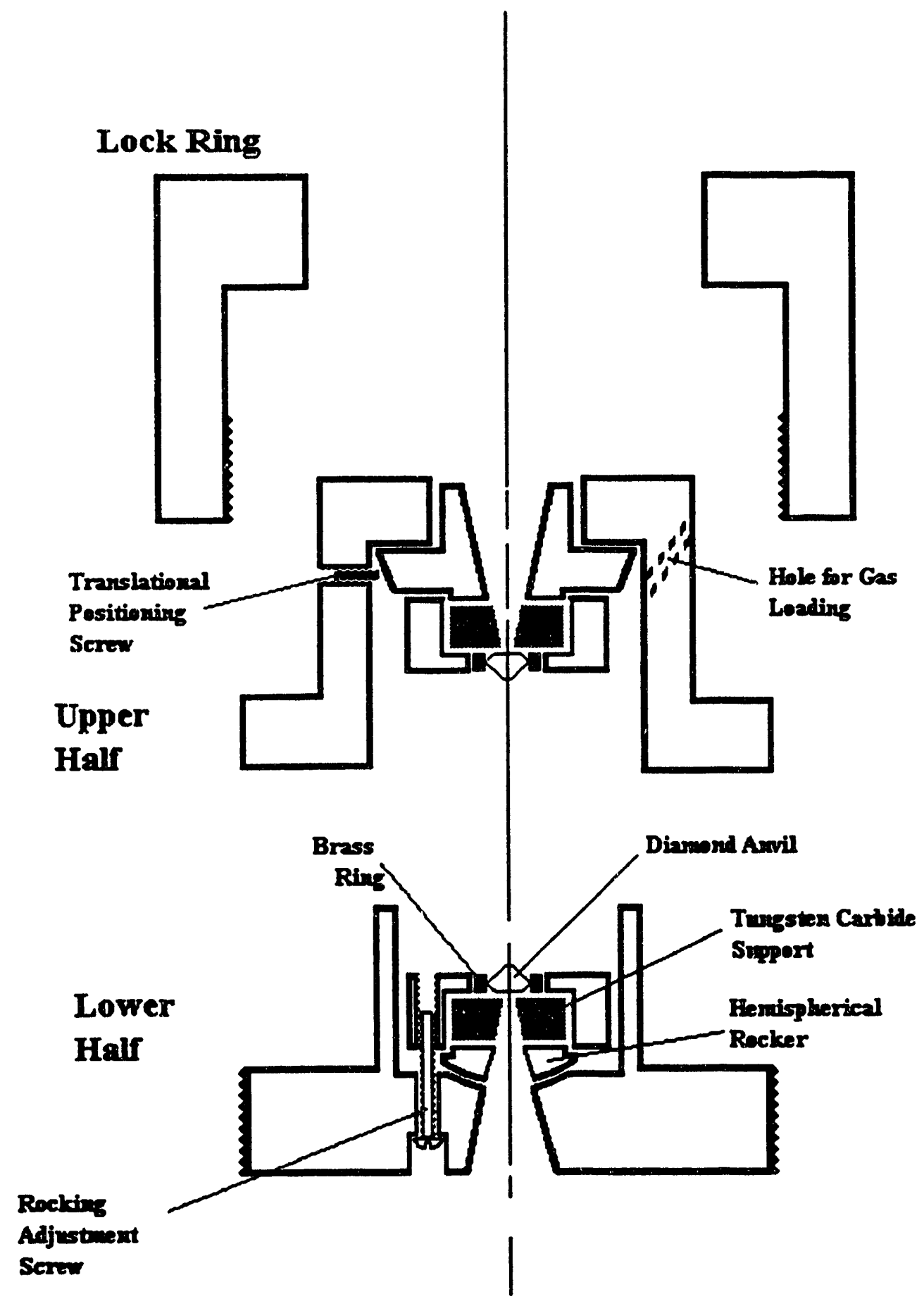

Fig. 4.1 


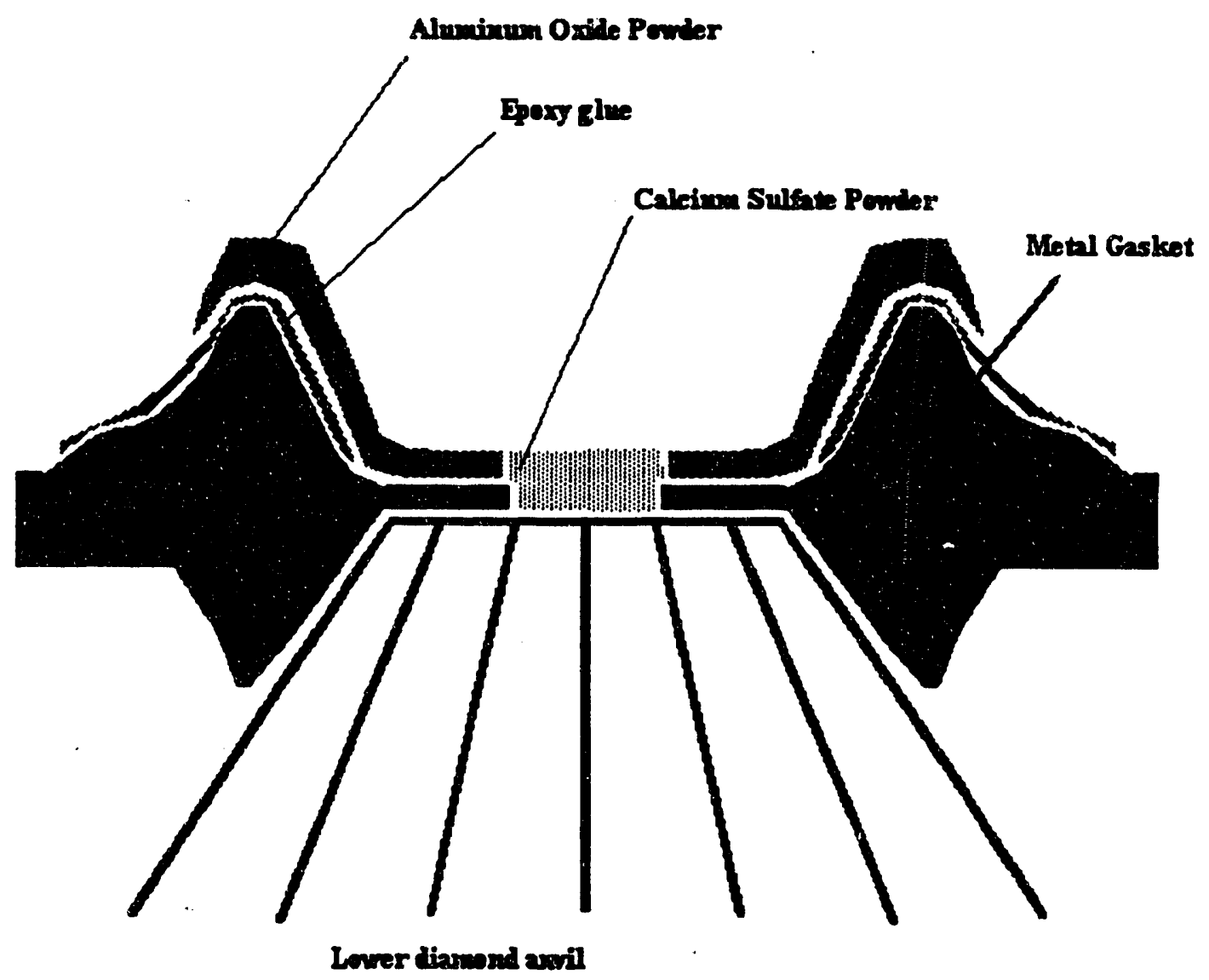

Fig. 4.2 


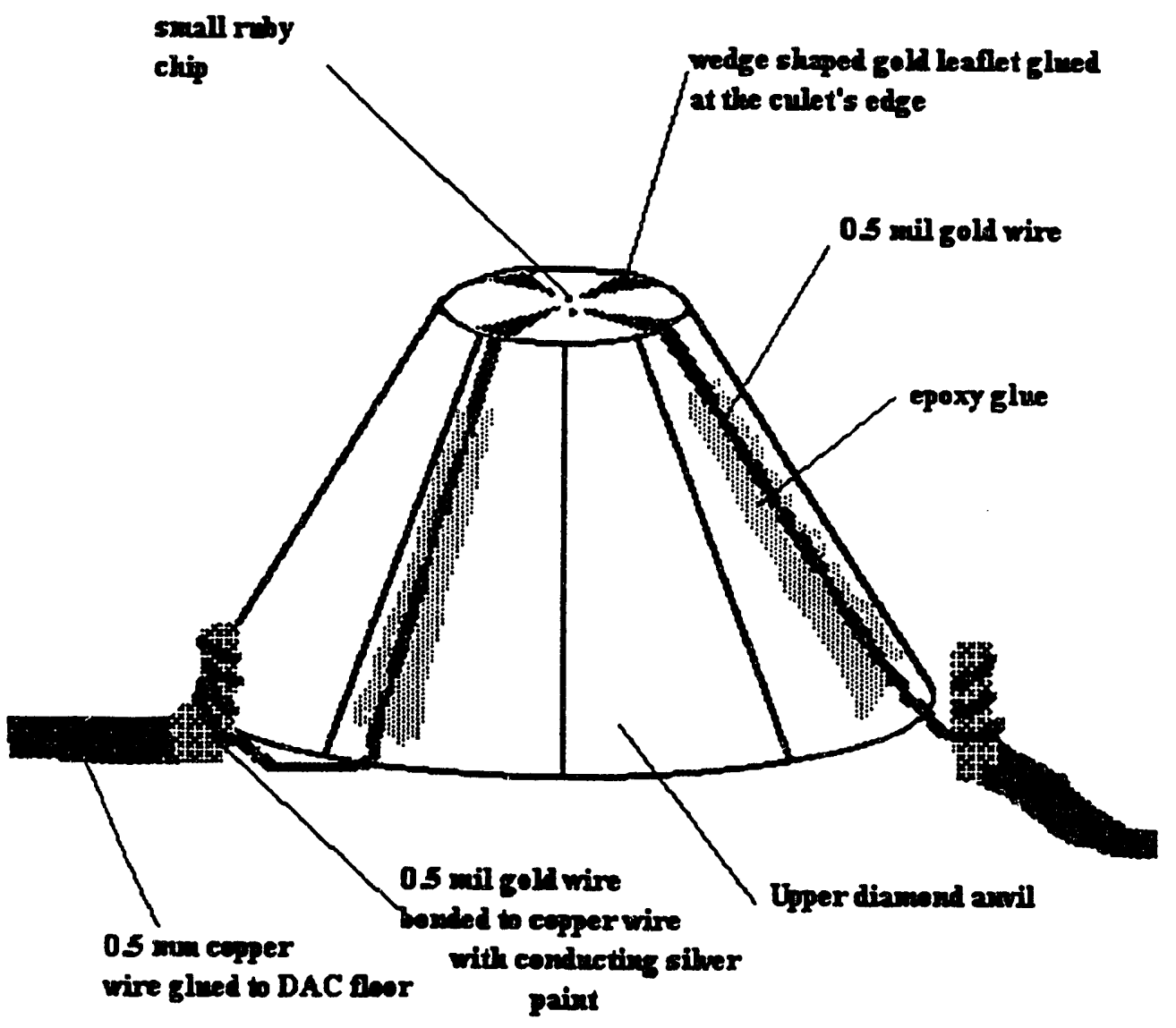

Fig. 4.3 


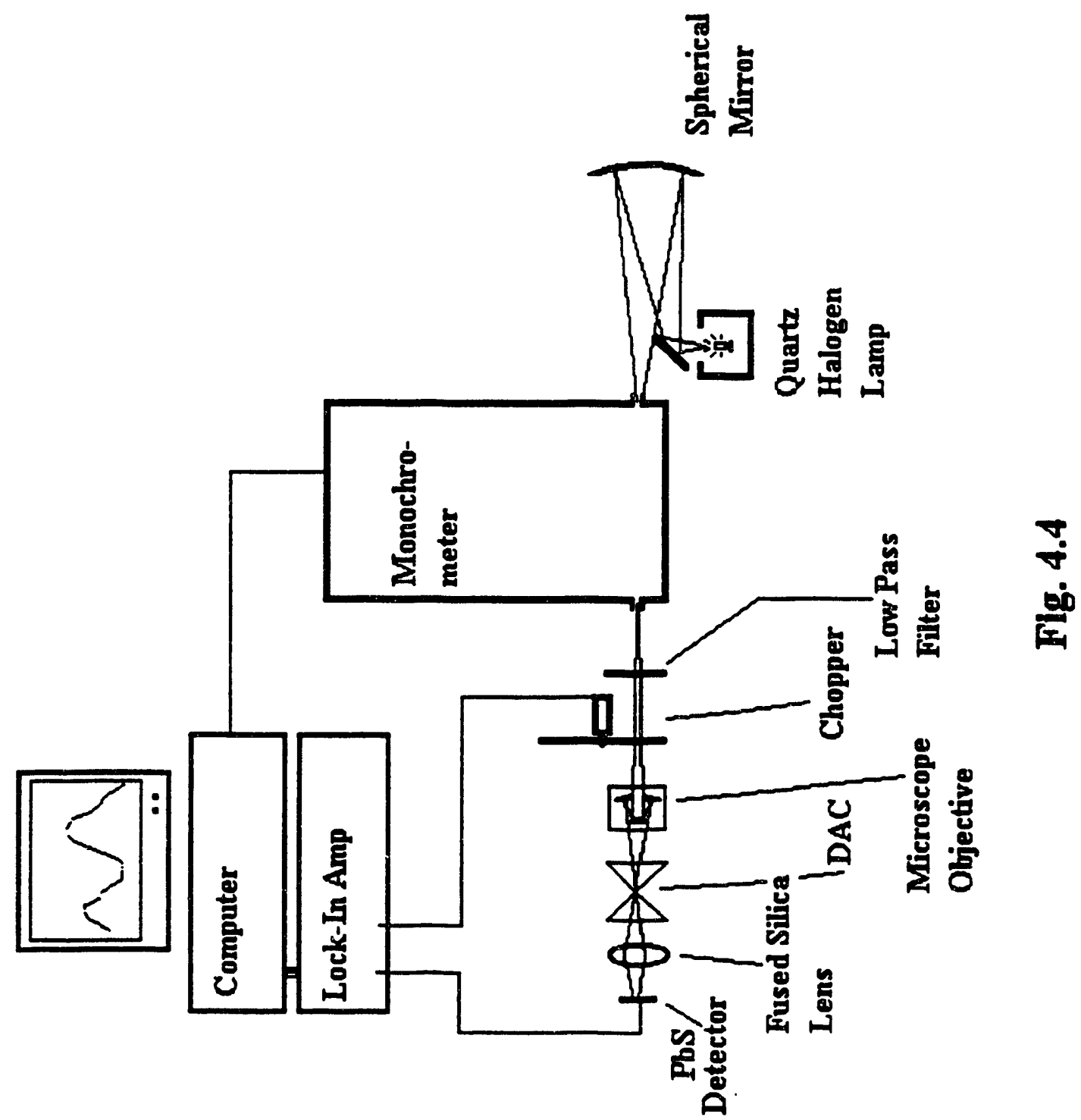




\section{Chapter V: High-Pressure Mössbauer and X-ray Diffraction Studies of $\mathrm{NiI}_{2}$ and $\mathrm{CoI}_{2}$ : Discovery of an Isostructural Magnetic Transition}

In this chapter we present and analyze Mössbauer spectroscopy and x-ray diffraction data taken on the transition-metal halides by our collaborators [37]. Actually, our interest in these compounds was triggered by observations of a pressure-induced quenching of the magnetic moments of $\mathrm{Ni}$ in $\mathrm{NiI}_{2}$ in the Mössbauer experiments performed by Moshe P. Pasternak and R. Dean Taylor. These additional measurements originally perked our interest in these compounds and provided vital information about the magnetic and crystalline structures respectively.

Mössbauer spectroscopy is one of the few ways to probe magnetic ordering under pressure inside a DAC. The Mössbauer effect is essentially the recoilless absorption of a $\gamma$ -ray by the nucleus of an atom within a crystal. The nuclear absorption line of a Mössbauer active nucleus is so narrow $\left(-10^{-9} \mathrm{eV}\right)$ that the splitting of this transition induced by a magnetic field of less than $1 \mathrm{kG}$ can easily be observed. When an antiferromagnetic or ferromagnetic crystal is in the ordered phase, the spin alignment produces an internal magnetic field. By measuring this magnetic-field strength as a function of temperature, one can determine the phase-transition temperature. Mössbauer spectroscopy can provide us with one additional piece of information. A shift in the center of the entire spectrum, the isomer shift, is a measure of the probability density of electrons at the Mössbauer nucleus and can provide information about changes in bonding between the Mössbauer nucleus and its neighbors.

When Mössbauer spectroscopy is applied to the detection of magnetic ordering, a non-magnetic ion is preferable as the Mössbauer nucleus. The spin of the magnetic ion will influence its own Mössbauer spectra even when the crystal is in the paramagnetic state. In $\mathrm{NiI}_{2}, \mathrm{CoI}_{2}$, and $\mathrm{FeI}_{2}$, the $\mathrm{I}-$ nucleus is situated at a high symmetry point so that the magnetic fields experienced by all the I- nuclei are non-zero and equal in magnitude 
when the transition-metal spins align antiferromagnetically. In other words, all the Mössbauer active iodine ions experience the same environment, and only one sharp spectrum results.

The source of the $\gamma$-rays for the experiment is a sample containing "hot" Mössbauer active 129I nuclei. The energy of the $\gamma$-rays from a source is scanned by the Doppler effect by moving the source at different constant velocities. The $\mathbf{x}$-axis of a Mössbauer spectra usually denotes the velocity of the source.

Representative Mössbauer spectra of the ${ }^{129} \mathrm{I}$ nucleus in $\mathrm{NiI}_{2}$ and $\mathrm{CoI}_{2}$ are shown in Fig. 5.1 and Fig. 5.3 respectively (data from ref.[37,38]). They are currently being performed on $\mathrm{FeI}_{2}$ as well. The $\mathrm{NiI}_{2}$ spectra show runs at various temperatures for a pressure of $6.7 \mathrm{GPa}$. The spectrum recorded at $185 \mathrm{~K}$ shows a single degenerate peak $\left(T T_{N}\right)$, while the others recorded at lower temperatures have multiple peaks due to splittings induced by the internal magnetic field $\left(T<T_{N}\right)$. The splittings increase with decreasing temperature. $T_{\mathrm{N}}$ was determined by extrapolating isobaric magnetic field vs. temperature data to zero field. Fig. 5.2 summarizes all the relevant information gathered from the Mössbauer data. $T_{\mathrm{N}}$ increases dramatically from $75 \mathrm{~K}$ at ambient pressures to $\sim 300 \mathrm{~K}$ at $19 \mathrm{GPa}$. The saturation hyperfine magnetic field is constant until $16 \mathrm{GPa}$ where it begins to decrease and disappears abruptly at $19 \mathrm{GPa}$. The smoothly varying isomer shift was interpreted as evidence of the absence of a discontinuous bonding change. Similar spectra were recorded for $\mathrm{CoI}_{2}$. But in this case, as Fig. 5.3 shows, the spectra are more complex. They show both a multiplet of peaks due to an antiferromagnetic phase and a single peak due to a non-magnetic phase for pressures between 9 and $12 \mathrm{GPa}$ suggesting that there is a coexistence of two phases. Fig. 5.4 sums up the $\mathrm{CoI}_{2}$ results. The relative fractions of the two components can be calculated from the Mössbauer spectra to give the fraction of the high-pressure phase, which is plotted in the inset. Furthermore, the two components have different isomer shifts, indicating a change in 
electronic configuration across the transition. In $\mathrm{CoI}_{2}$ the increase in $T_{\mathrm{N}}$ is more dramatic, rising from $10 \mathrm{~K}$ to $150 \mathrm{~K}$ with an increase in pressure of $10 \mathrm{GPa}$.

It is evident from the increase in $T_{\mathrm{N}}$ with pressure in $\mathrm{NiI}_{2}$ and $\mathrm{CoI}_{2}$ that as pressure is increased, the antiferromagnetic phase in both compounds first increases in stability and then disappears abruptly. We do not know completely the magnetic properties of the high-pressure phases. All we know is that there is no magnetic ordering down to about 4 $\mathrm{K}$ in the high-pressure phase so we will refer to them as non-magnetic phases. When this phase was first discovered, the underlying cause of this transition was not known. An $\mathrm{x}$ ray diffraction study was performed with a rotating anode source to check for structural changes [37]. The resultant volume and lattice constants, $c$ and $a$, as a function of pressure are strown in Figs. 5.5 and 5.6 respectively [37,39]. No change in crystal structure or measurable discontinuity in the lattice constants was observed, suggesting that the magnetic to non-magnetic transition in $\mathrm{NiI}_{2}$ is purely electronic. High-pressure $\mathrm{x}$-ray studies on $\mathrm{CoI}_{2}$ and $\mathrm{FeI}_{2}$ are currently being performed. 


\section{Figure Captions for Chapter V}

Fig. 5.1 Representative Mössbauer spectra of $\mathrm{Nil}_{2}$ at $6.7 \mathrm{GPa}$ of pressure. The amount of splitting of the peak with decreasing temperature is proportional to the internal magnetic field. Above the Néel temperature the peak is not split. Ref. [37,38]

Fig. 5.2 Néel temperature, saturation magnetic field, and isomer shift of $\mathrm{NiI}_{2}$ as determined from the Mössbauer spectra. $T_{\mathrm{N}}$ rises from $\sim 75$ to $\sim 300 \mathrm{~K}$. The saturation field disappears above $19 \mathrm{GPa}$ indicating a magnetic phase transition to a non-magnetic phase. The increase in the isomer shift with pressure is smooth, suggesting that there is no discontinuous change in bonding. Ref. [37,38].

Fig. 5.3 Low-temperature Mössbauer spectra of $\mathrm{CoI}_{2}$ at various pressures. In addition to the magnetic-field split lines, the 9.5 and $11.8 \mathrm{GPa}$ spectra show coexistence of two phases, one with (antiferromagnetic phase) and one without (non-magnetic) peak splittings. At $13.0 \mathrm{GPa}$ only the non-magnetic phase exists. Ref. [39].

Fig. 5.4 The pressure dependence of the Néel temperature (c), saturation magnetic field (b), and isomer shift (a) of $\mathrm{CoI}_{2}$ as determined from the Mössbauer spectra. Notice that at low (high) pressures only the antiferromagnetic (non-magnetic) phase exists. However, between 9.5 and $12 \mathrm{GPa}$ the two phases coexist. The non-magnetic $\mathrm{CoI}_{2}$ has a different isomer shift, suggesting a change in bonding. The fraction of non-magnetic phase as a function of pressure is graphed in the inset. Ref. [38].

Fig. 5.5 Volume vs. pressure data of $\mathrm{NiI}_{2}$ determined by $\mathrm{x}$-ray diffraction. There is no evidence of a structural phase transition or discontinuous change in volume. Ref. [37]. 
Fig. 5.6 The pressure dependence of the $c$ and $a$ lattice constants of $\mathrm{NiI}_{2}$ as determined by $\mathrm{x}$-ray diffraction. There is no sign of discontinuity. Lines are third-order least-squares fits to the data. Ref. [39]. 


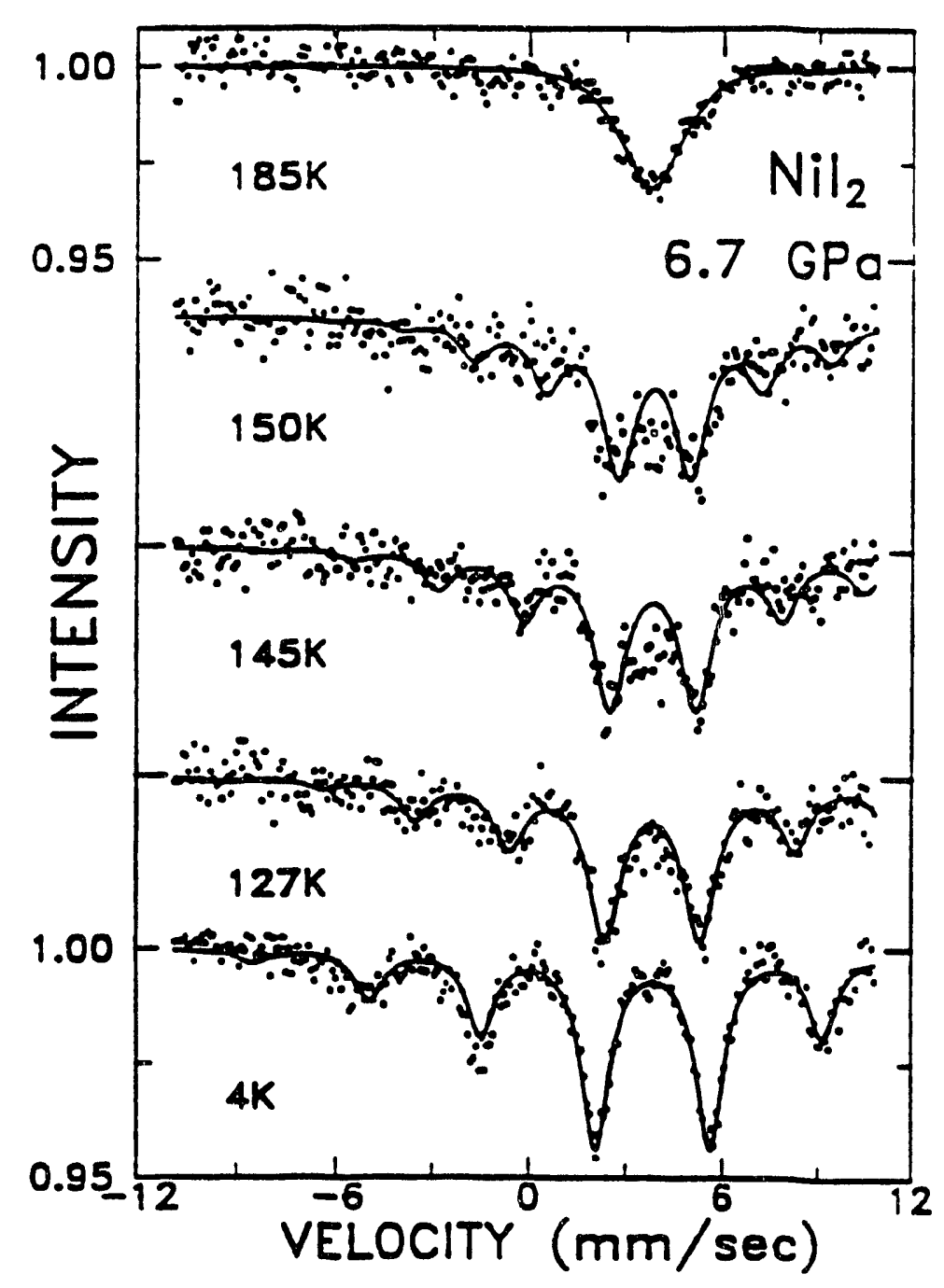

Fig. 5.1 


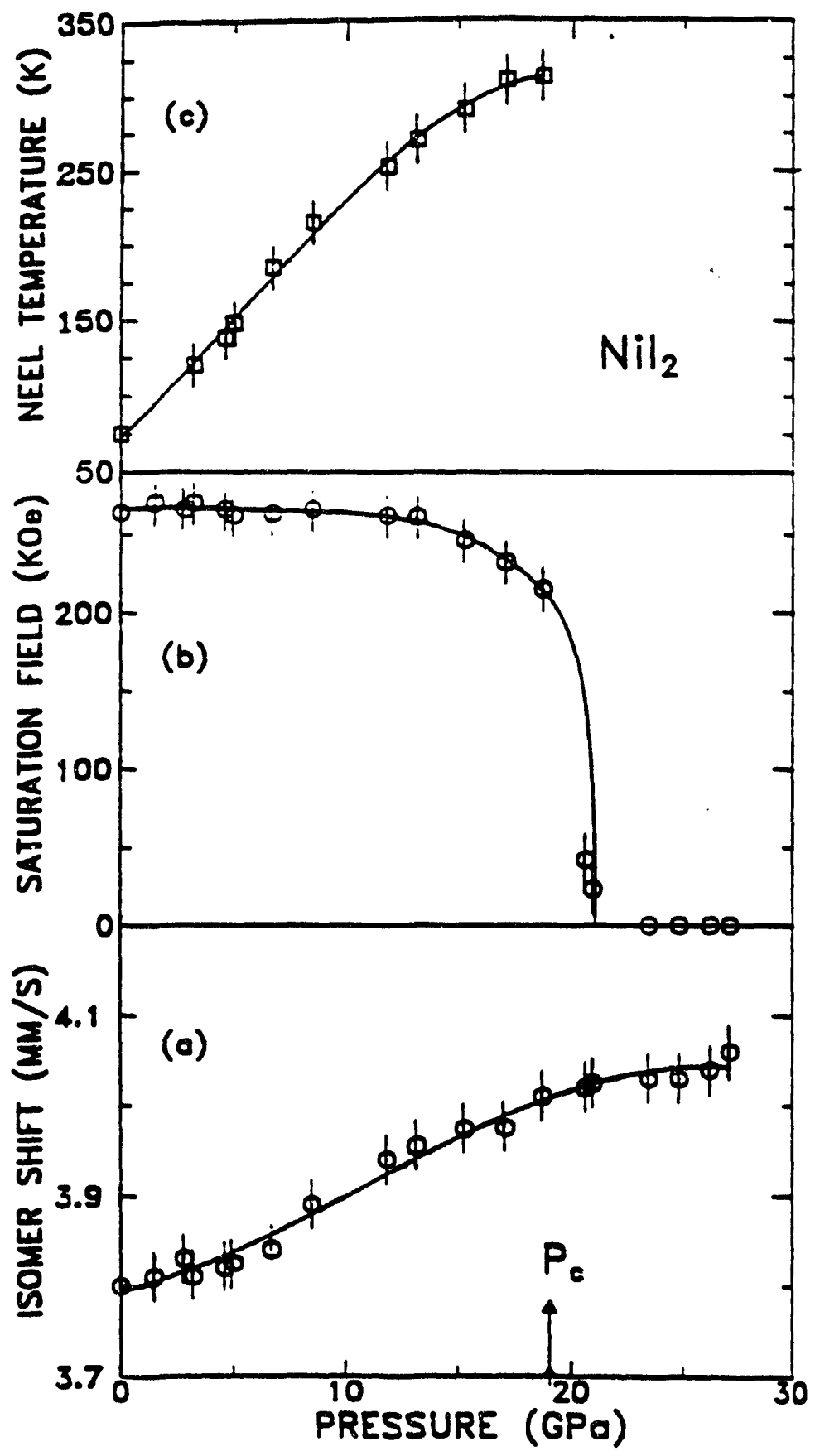

Fig. 5.2 


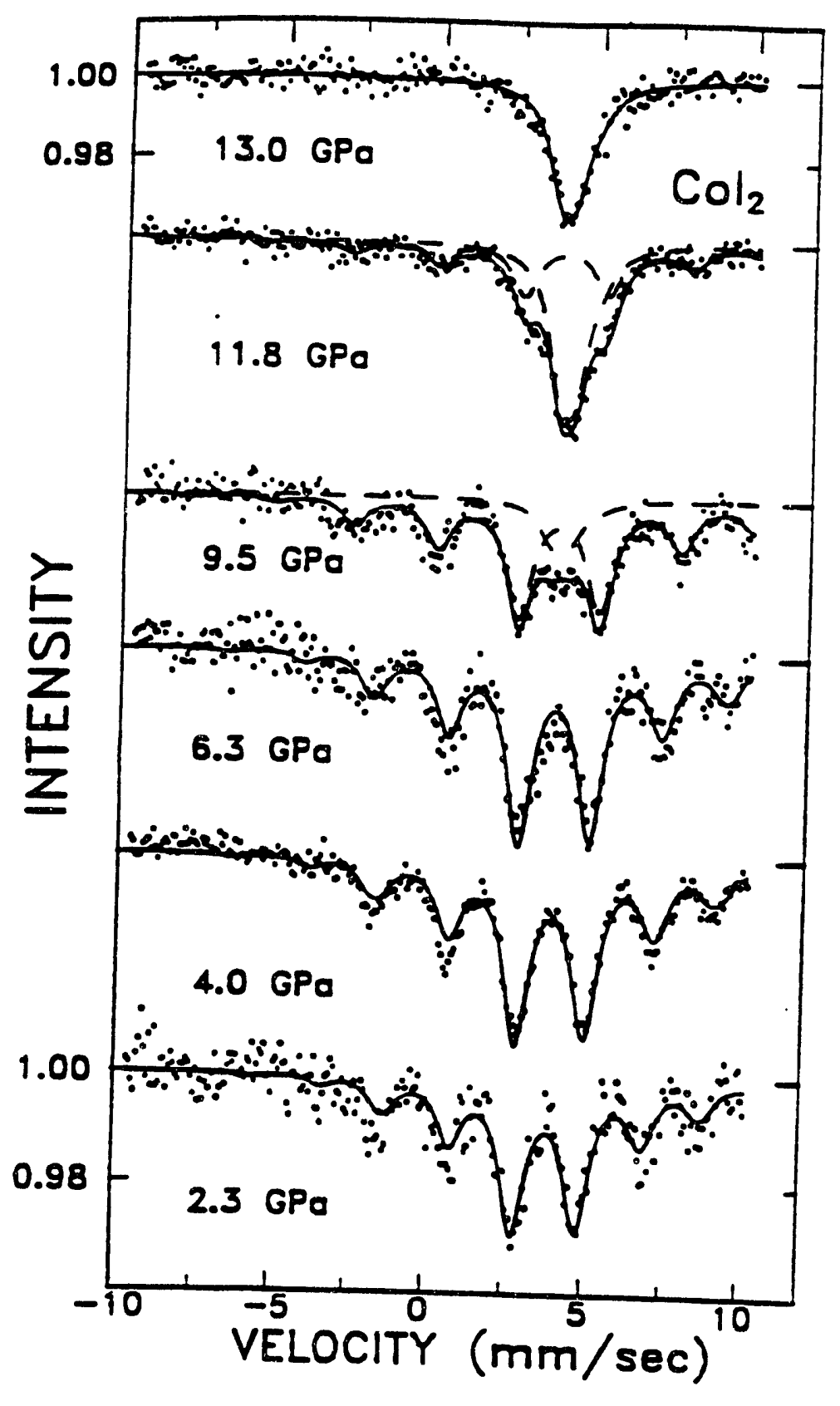

Fig. 5.3 


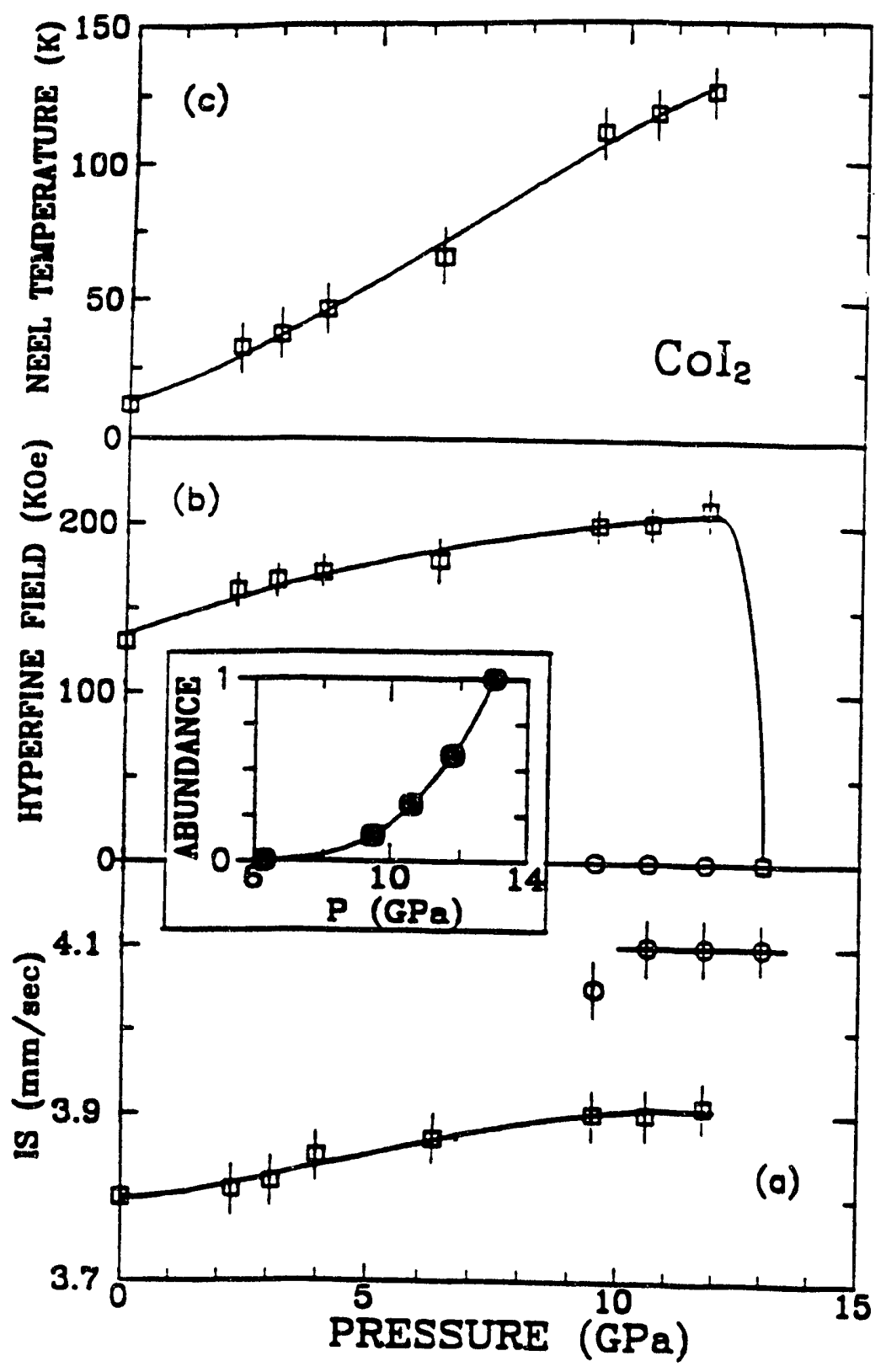

Fig. 5.4 


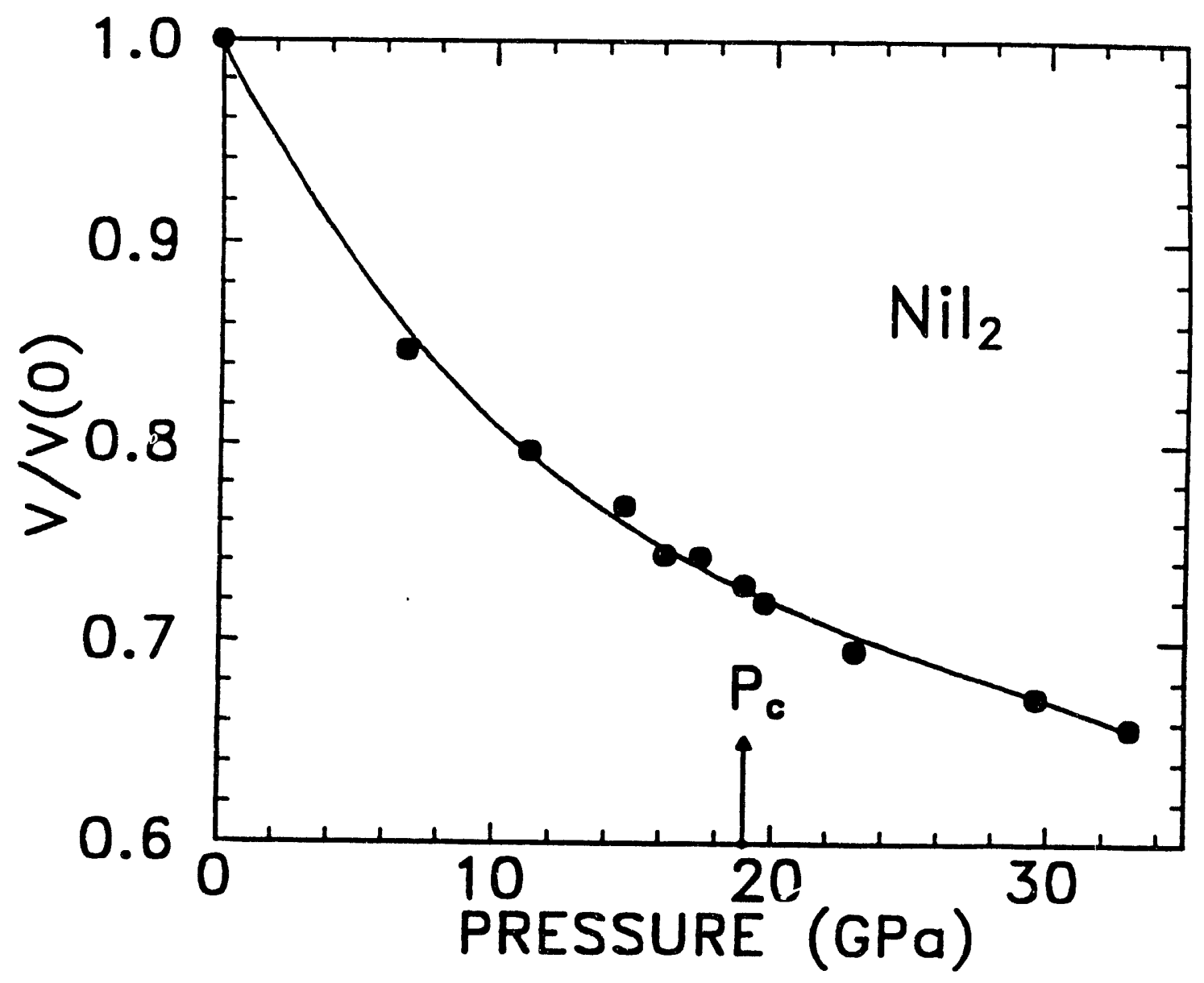

Fig. 5.5 


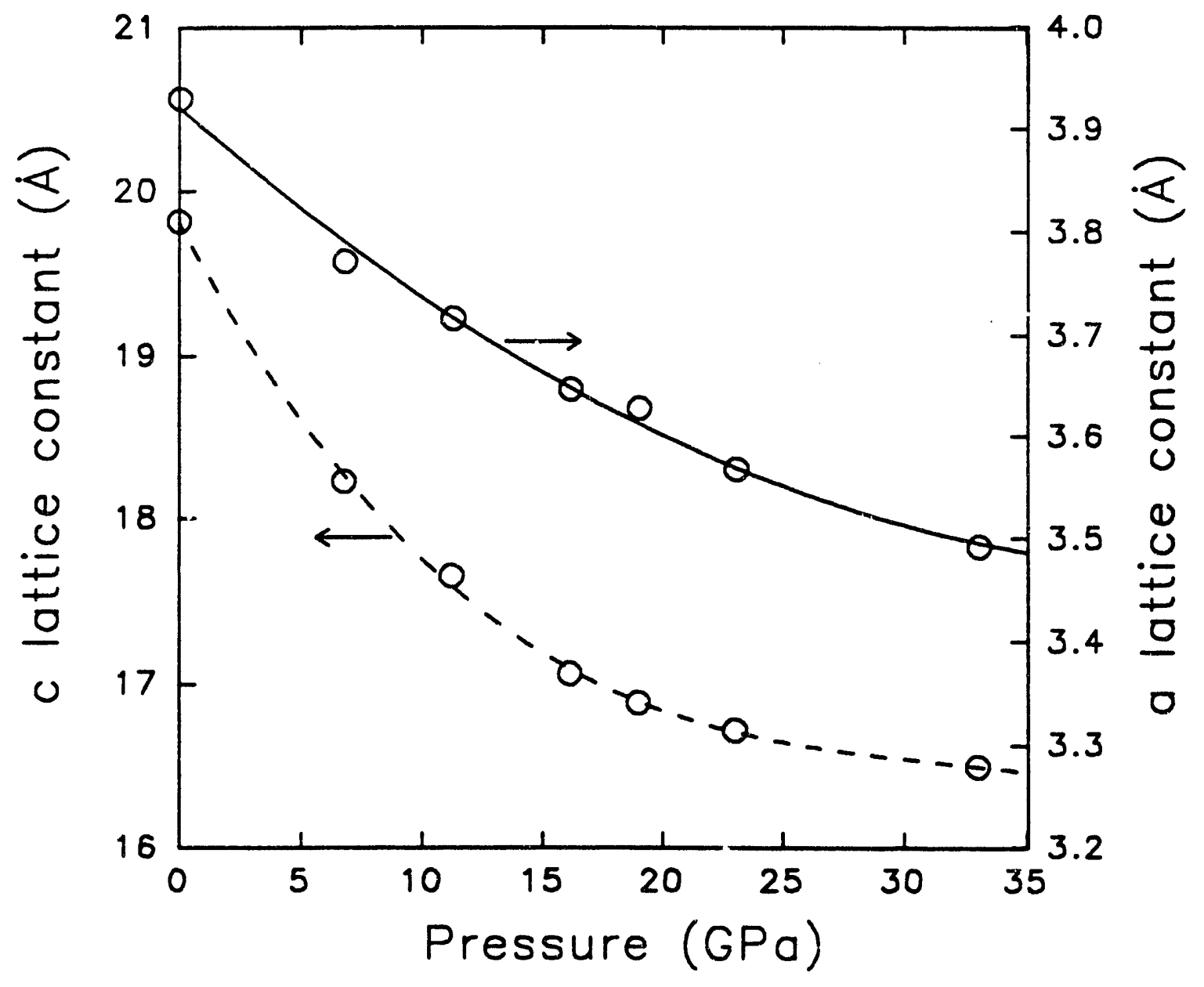

Fig. 5.6 


\section{Chapter VI: Resistivity Data and Analysis}

In this charter we present the resistivity measurements performed under high pressure on $\mathrm{NiI}_{2}, \mathrm{CoI}_{2}$, and $\mathrm{FeI}_{2}$. Our main interest in pursuing these measurements was to determine whether an IM transition occurs in these materials in the neighborhood of the reported magnetic transition. From the resistivity measurements we determined that these compounds do become metallic under pressure by gradual closure of an activation energy. Section A describes the $\mathrm{NiI}_{2}$ resistivity data and its analysis. In section $\mathrm{B}$ we present similar data on $\mathrm{CoI}_{2}$ and $\mathrm{FeI}_{2}$.

\section{A: Resistivity Data of $\mathrm{NiI}_{2}$}

The results of our first resistance study on $\mathrm{NiI}_{2}$ are shown in Fig. 6.1 [37]. This is a quasi-four probe measurement; the reported resistance includes the contact resistance in series with the sample resistance. The data were taken at $300 \mathrm{~K}$ with increasing pressure. The resistance decreases exponentially with increasing pressure for pressures below 14 GPa. Above $19 \mathrm{GPa}$ the conductivity saturates and the resistivity levels off. The resistance as a function of temperature is plotted in the inset of the graph for two pressures, 17 and $21 \mathrm{GPa}$. In the $17 \mathrm{GPa}$ run the resistance increases with decreasing temperature, indicating the existence of thermally activated carriers and implying semiconducting behavior. However the $21 \mathrm{GPa}$ run has a resistance that increases with increasing temperature, suggesting that scattering is dominated by phonons as is the case in most metals.

In addition to the temperature-dependent measurements, the exponential decrease in the resistance and its eventual leveling also suggest that pressure induces an $\mathrm{IM}$ transition. Furthermore, these results indicate that closure of an activation energy gap causes metallization as discussed in Chapter III. These preliminary results suggest that metallization in $\mathrm{NiI}_{2}$ occurs between 17 and $19 \mathrm{GPa}$. 
In this earlier experiment, the resistance was also measured as a function of decreasing pressure (results not plotted) to search for any hysteresis in the IM transition. For any phase transition, a natural question is whether it is a first-or second-order phase transition. If th - phase transition is strongly first-order, one would expect to see some hysteresis in the transition. This occurs because of slow kinetics of the transformation and internal stresses at the boundary of the two phases during the transformation [39a]. The pressure-induced, first-order, structural $\mathrm{MM}$ transitions in the III-V semiconductors show hysteretic resistance as a function of pressure. The measurements of $\mathrm{NiI}_{2}$ do not indicate any noticeable hysteresis. Of course these measurement do not preclude that the IM transition in $\mathrm{NiI}_{2}$ is first-order, but rather suggest that the transition is either second-order or weakly first-order. Upon decreasing the pressure the resistance increased but did not return to its original low pressure value. This is expected because in the resistance measurements the pressure is only quasi-hydrostatic. A large component of uniaxial stress may cause the sample to be permanently deformed.

Later, we repeated the resistance measurements with several improvements[40]. i) We used a four-probe technique to measure the true resistance of the sample. We found that, for our samples, the contact resistance was typically 10 times higher than the sample resistance, demonstrating the need for a true four-probe measurement. ii) From the sample size $\left(50 \times 50 \times 10 \mu \mathrm{m}^{3}\right)$ and the resistance, we were able to estimate the resistivity of our sample and determine the metallic conductivity at high pressure. iii) A more thorough temperature-dependent study was performed. Arrhenius plots of the temperaturedependent resistivity in the insulating state allowed us to determine the carrier-activation energy as a function of pressure.

Data from the improved measurements are shown in Fig. 6.2 and 6.3. One can see from the pressure-dependent resistivity that the general features are the same as in Fig. 6.1. In the region below $15 \mathrm{GPa}$ the resistivity decreases exponentially. Above $18 \mathrm{GPa}$ the resistivity levels and becomes comparatively independent of pressure. Around 16.5 
$\mathrm{GPa}$, however, a distinct knee appears in the resistivity which is reproducible over several runs using different $\mathrm{NiI}_{2}$ samples. A closer examination of the data in Fig. 6.1 shows that this knee is also present at approximately the same pressure. This knee is suggestive of a slight discontinuity in the resistivity that is smoothed over by the pressure inhomogeneity, implying that the transition may be electronically first-order. We shall retum to this interesting point later in the discussions.

The temperature-dependent resistivity at various pressures is plotted in an Arrhenius format in Fig. 6.3 [40]. When the activation energy is large in comparison to the temperature, the resistivity is determined by the expression

$$
\rho=\rho_{0} \exp \left(\frac{E_{t}}{2 k T}\right)
$$

Here $\rho_{0}$ is the high temperature resistivity and is inversely proportional to the mobility $(\mu)$. The activation energy, $E_{t}$, can be determined by the Arrhenius plots. For runs at pressures below $16.2 \mathrm{GPa}$ the resistivity exhibits activated behavior. The slopes of the Arrhenius plots in this pressure range are not constant but rather suggest a range of activation energies. The activation energies are deduced from the resistivity curves in Fig. 6.3 using Eq. (6.1) provided that $\rho_{0}$ is indeed temperature independent. First we must examine when the temperature dependence of the mobility can be neglected. The logarithmic derivative of Eq. (6.1) with respect to temperature is

$$
\frac{d \ln (\rho)}{d \ln (T)}=\frac{E_{t}}{2 k T}-\frac{d \ln (\mu)}{d \ln (T)}
$$

The temperature dependence of the mobility in $\mathrm{NiI}_{2}$ is not known, but in intrinsic semiconductors where $\mu$ is dominated by electron-phonon scattering $d \ln (\mu) / d \ln (T) \sim-2.5$ so that it can safely be ignored when $E_{t} \gg 2 k T$. Then $E_{l}$ can be determined by the slope of the Arrhenius plot.

$$
E_{t}=2 k \frac{d \ln (\rho)}{d(1 / T)}
$$


The values of $E_{t}$ determined in this way for $\mathrm{NiI}_{2}$ are shown as open squares in Fig. 6.4. The vertical bars at each data point in Fig. 6.4 indicate the range of activation energies deduced from the Arrhenius plots. At a pressure of $5 \mathrm{GPa}, E_{t}$ is on the order of $0.5 \mathrm{eV}$ and decreases with pressure at an initial rate of $\sim-110 \mathrm{meV} / \mathrm{GPa} . E_{t}$ can be extrapolated back to an atmospheric pressure value of about $1.1 \mathrm{eV}$ which is smaller than the chargetransfer gap. With increasing pressure, the rate of decrease slows above $10 \mathrm{GPa}$ and extrapolates to zero around $16-17 \mathrm{GPa}$. In the inset of Fig. 6.2 we show the resistivity of $\mathrm{NiI}_{2}$ versus temperature on a linear scale at 16.2 and $19.3 \mathrm{GPa}$. In both curves the resistivity increases almost linearly with temperature, as expected for a metal except for the small dip at very low temperature in the $16.2 \mathrm{GPa}$ curve.

$d E_{t} d P$ can also be estimated from a quantitative analysis of the resistivity vs. pressure data (Fig. 6.1 and 6.2) using Eq. (6.1). If $\rho_{0}$ is assumed to be independent of pressure and $E_{t}=\alpha\left(P-P_{0}\right)$ for $P<P_{0}$, the resistivity would show an exponential decrease as a function of pressure for pressures below $P_{0}$, the gap closure pressure, as discussed above. The coefficient $\alpha=d E_{t} / d P$, when determined from the slope of the data points taken at pressures below $12 \mathrm{GPa}$ in Fig. 6.2 , is $\sim-50 \mathrm{meV} / \mathrm{GPa}$, roughly half the value of $d E_{t} / d P$ determined by the Arrhenius plots. Part of the difference can be attributed to the pressure dependence of $\rho_{0}$. Since our pressure medium is not hydrostatic, defects may be created at high pressure causing $\rho_{0}$ to have a strong pressure dependence. Nevertheless, we note that the values of $d E_{\downarrow} / d P$ deduced from both sets of data are roughly the same order of magnitude.

There is another criterion for determining whether a conducting material is metallic. Mott and others calculated the minimum conductivity of a metal based on the fact that the mean free path of an electron can never be less than the distance between the ions. Their estimate of the minimum metallic conductivity is $\sim 10^{3} \mathrm{ohm}^{-1}-\mathrm{cm}^{-1}[2]$. At 18 $\mathrm{GPa}$ we estimate that the conductivity of $\mathrm{NiI}_{2}$ is about $2 \times 10^{3} \mathrm{ohm}^{-1}-\mathrm{cm}^{-1}$, indicating that it is possible that the material has reached a metallic state. 
All our data unambiguously show that metallization of $\mathrm{NiI}_{2}$ occurs around $17 \mathrm{GPa}$ with an uncertainty of $\pm 1 \mathrm{GPa}$. Firstly, the pressure-dependent resistivity measurements at room temperature show a leveling of the resistivity above $17 \mathrm{GPa}$ (Fig. 6.1 and 6.2). Secondly, the temperature dependence of the resistivity shows a crossover from semiconducting to metallic behavior for pressures between 16 and $19 \mathrm{GPa}$ (inset in Fig. 6.2). Finally the conductivity reaches the minimum metallic value in that range. Furthermore the measurements indicate metallization by closure of a transport energy gap. In general the changes in the transport properties under pressure happen smoothly. There are no precipitous drops in resistivity of several orders of magnitude, as often occurs when there is a first-order structural change. This is consistent with the $x$-ray study reviewed in the previous chapter which rules out any structural transformations and any large discontinuity in the lattice constants.

\section{B: Resistivity Data of $\mathrm{CoI}_{2}$ and $\mathrm{FeI}_{2}$}

In this section we present resistivity measurements of $\mathrm{CoI}_{2}$ made by some of our collaborators [41] and our own measurements on $\mathrm{FeI}_{2}$. The measurements on $\mathrm{CoI}_{2}$ show that it undergoes an $\mathrm{MM}$ transition near $10 \mathrm{GPa}$ where the magnetic transition begins. $\mathrm{FeI}_{2}$ also metallizes but at higher pressures $(-23 \mathrm{GPa})$. These measurements underscore the fact that gap closure $\mathbb{M}$ transitions and associated the magnetic transitions are general high-pressure properties of these materials.

The room-temperature resistance as a function of pressure for $\mathrm{CoI}_{2}$ is plotted in Fig. 6.5. It again shows an exponentially decreasing resistivity that levels off at high pressures, the signature of a closing activation gap. There also seems to be a knee, in this case even more pronounced than in $\mathrm{NiI}_{2}$, starting at $8 \mathrm{GPa}$ and continuing up to $10 \mathrm{GPa}$. This knee may suggest a discontinuous closure of the carrier-activation gap although further detailed studies would be necessary to strengthen this conclusion. The metallization occurs at about $10 \pm 1 \mathrm{GPa}$, as determined from Fig. 6.5. Temperature- 
dependent measurements (not shown) were also performed and they confirm a change from semiconducting to metallic behavior at $10 \mathrm{GPa}$. Our resistivity data on $\mathrm{FeI}_{2}$ are shown in Fig. 6.6, which indicate metallization at about $23 \pm 1 \mathrm{GPa}$. They, however, do not have the characteristic knee that is apparent in the both $\mathrm{CoI}_{2}$ and $\mathrm{NiI}_{2}$. Although we have not carried out temperature-dependent measurements on $\mathrm{FeI}_{2}$, results similar to those in $\mathrm{NiI}_{2}$ and $\mathrm{CoI}_{2}$ are expected.

We made a rough estimate of $d E_{t} d P$ for both of these compounds using the slope of the $\log (\mathrm{R})$-versus-P curves at low pressure assuming that pressure does not greatly affect the mobility just as we did for $\mathrm{NiI}_{2}$. For $\mathrm{CoI}_{2}$ this is the region below $8 \mathrm{GPa}$. The result is $\sim-120 \mathrm{meV} / \mathrm{GPa}$ for $\mathrm{CoI}_{2}$ and $\sim-34 \mathrm{meV} / \mathrm{GPa}$ for $\mathrm{FeI}_{2}$. Mössbauer measurements on $\mathrm{FeI}_{2}$ are underway and it is quite likely that a magnetic transition around the metallization pressure of $23 \mathrm{GPa}$ will be observed. 


\section{Figure Captions for Chapter VI}

Fig. 6.1 Quasi-four probe resistance of $\mathrm{NiI}_{2}$ as a function of pressure or volume change at $300 \mathrm{~K}$. The main features to note are a resistance that decreases exponentially up to 15 $\mathrm{GPa}$ a conspicuous knee at $17 \mathrm{GPa}$, and a relatively constant resistance above $19 \mathrm{GPa}$. The inset shows the temperature dependence of the resistance at a) $17 \mathrm{GPa}$ and b) 21 $\mathrm{GPa}$.

Fig. 6.2 True four-probe resistivity measurements of $\mathrm{NiI}_{2}$ vs. pressure at $300 \mathrm{~K}$. The resistivity was estimated from the resistance of the sample using its estimated size and geometry. Inset shows temperature dependence of the resistivity plotted on a linear scale at $16.2 \mathrm{GPa}$ and $19.3 \mathrm{GPa}$. The resistivity at the latter pressure has been magnified 10 times.

Fig. 6.3 Temperature-dependent resistivity data of $\mathrm{NiI}_{2}$ at various pressures plotted in Arrhenius format. The curves with pressures below $16 \mathrm{GPa}$ show activated behavior. The 19.3 GPa run shows metallic behavior. The $16.2 \mathrm{GPa}$ run near the metallization point shows both activated and metallic behavior.

Fig. 6.4 Activation energies vs. pressure for $\mathrm{NiI}_{2}$. The error bars represent the range of activation energies exhibited by the Arrhenius plots. Lines are a guide for the eye. The activation energies decrease monotonically and extrapolate to a metallization pressure of 16-17 GPa.

Fig 6.5 Resistance-vs.-pressure data for $\mathrm{CoI}_{2}$ (from Ref. [41]). Data were taken using a quasi-four probe technique at $300 \mathrm{~K}$. Notice the same features as in Fig. 6.1. The 
resistance decreases exponentially upto $8 \mathrm{GPa}$, where it begins to decrease more steeply (a large knee), and then levels off above 10. GPa.

Fig. 6.6 Resistivity vs. pressure of $\mathrm{FeI}_{2}$ to $32 \mathrm{GPa}$. Data were taken with a true fourprobe technique at $300 \mathrm{~K}$. Leveling of the resistivity at pressures above $23 \mathrm{GPa}$ suggests that metallization occurs at that pressure. 


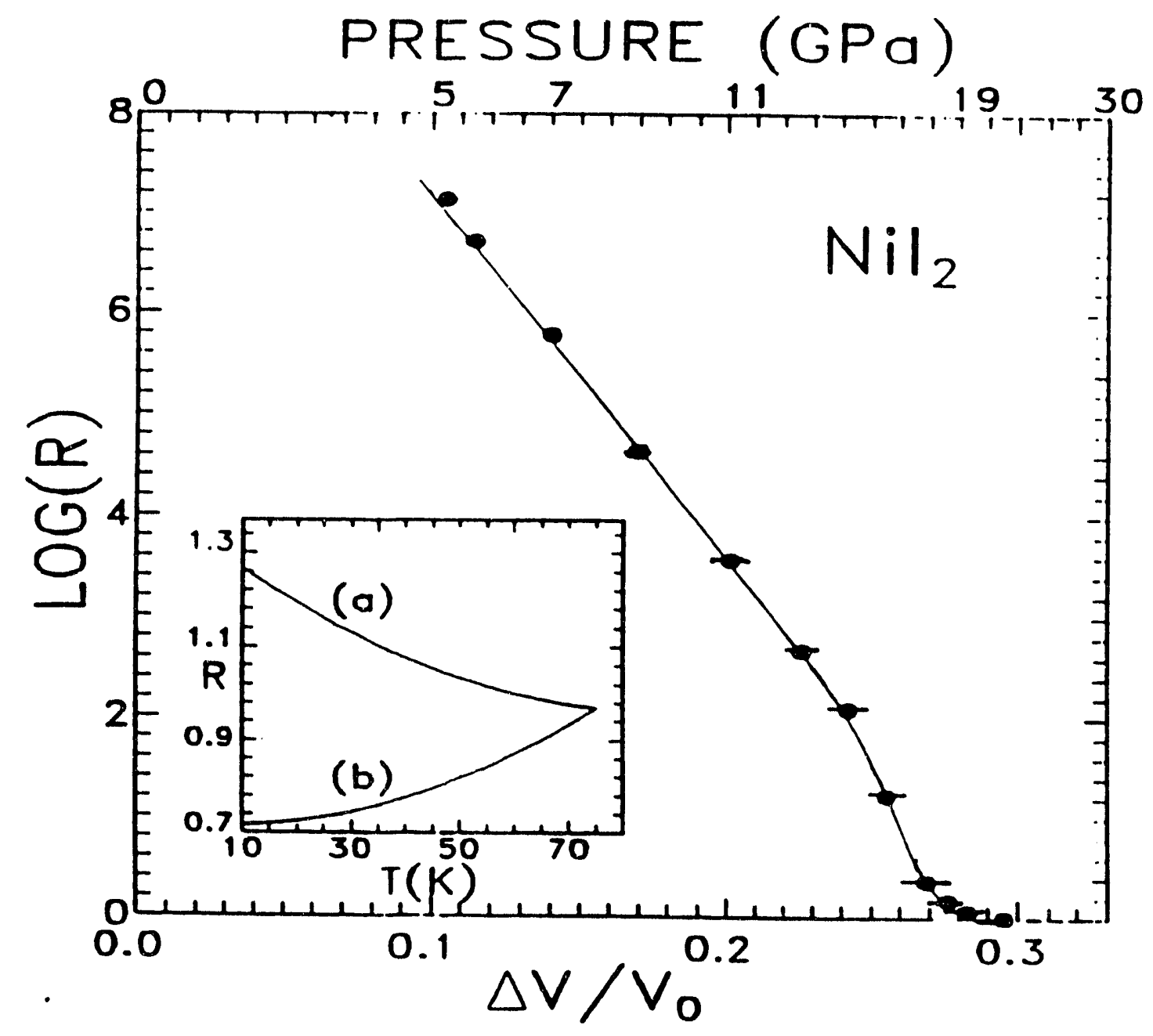

Fig. 6.1 


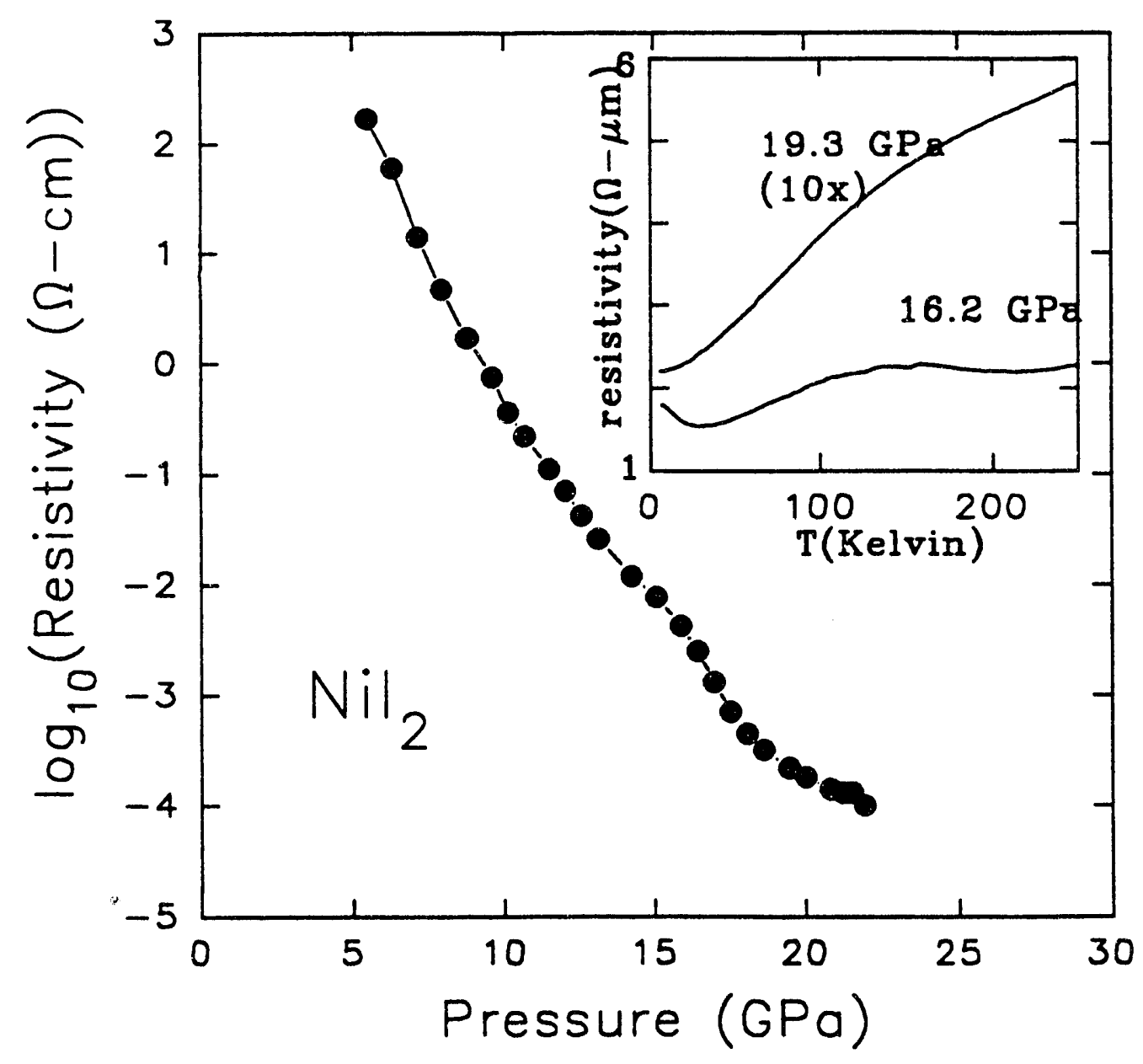

Fig. 6.2 


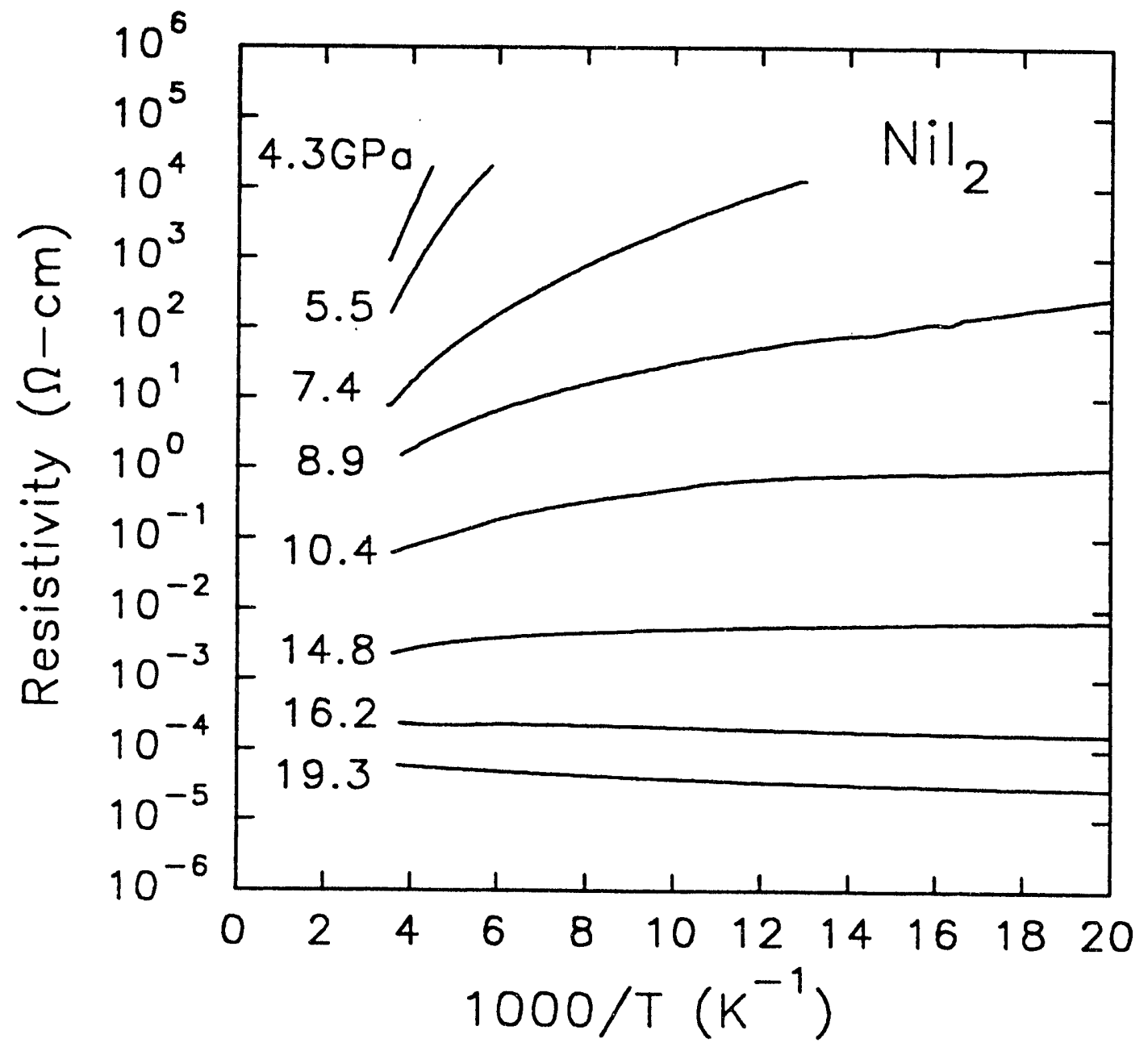

Fig. 6.3 


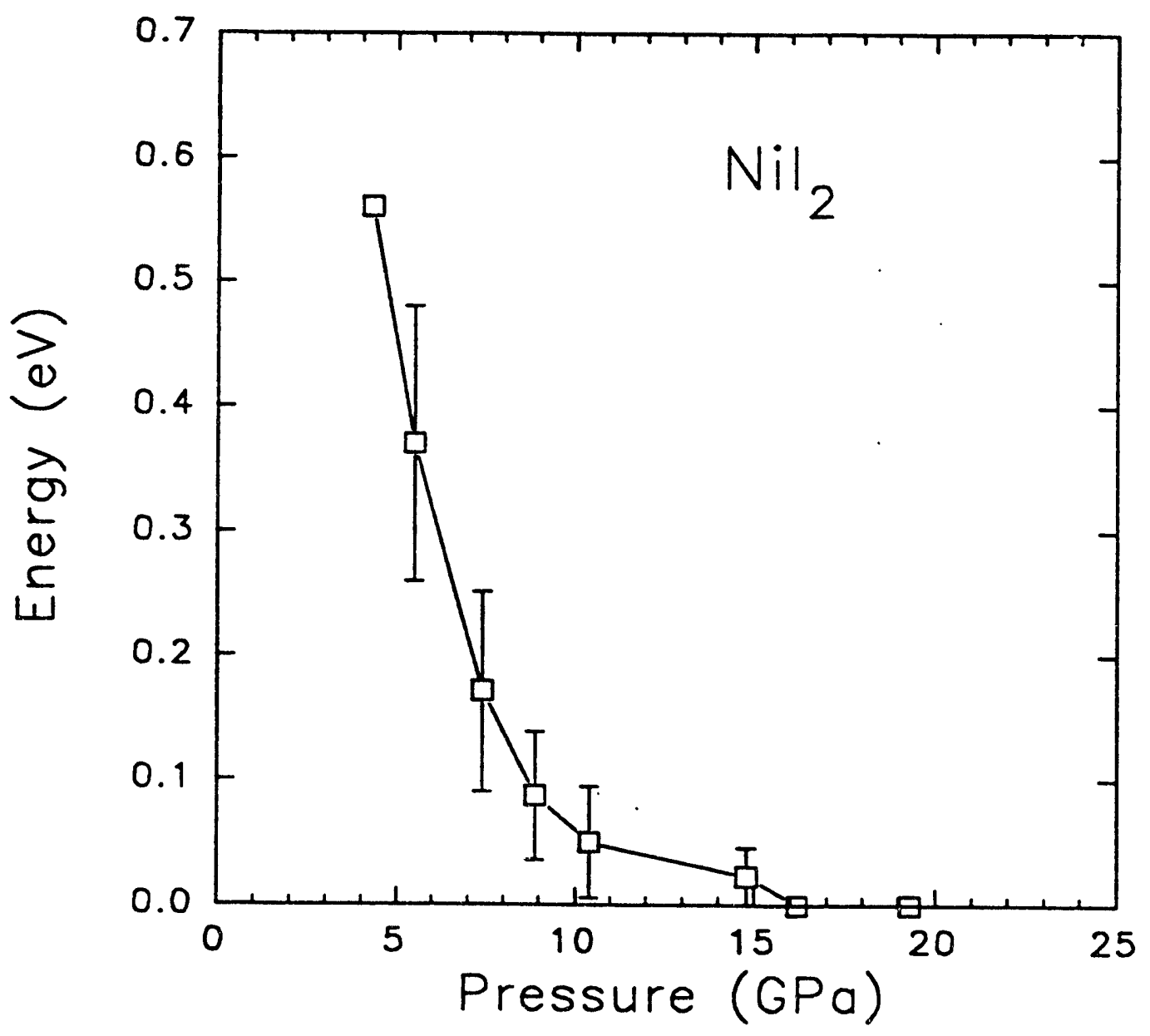

Fig. 6.4 


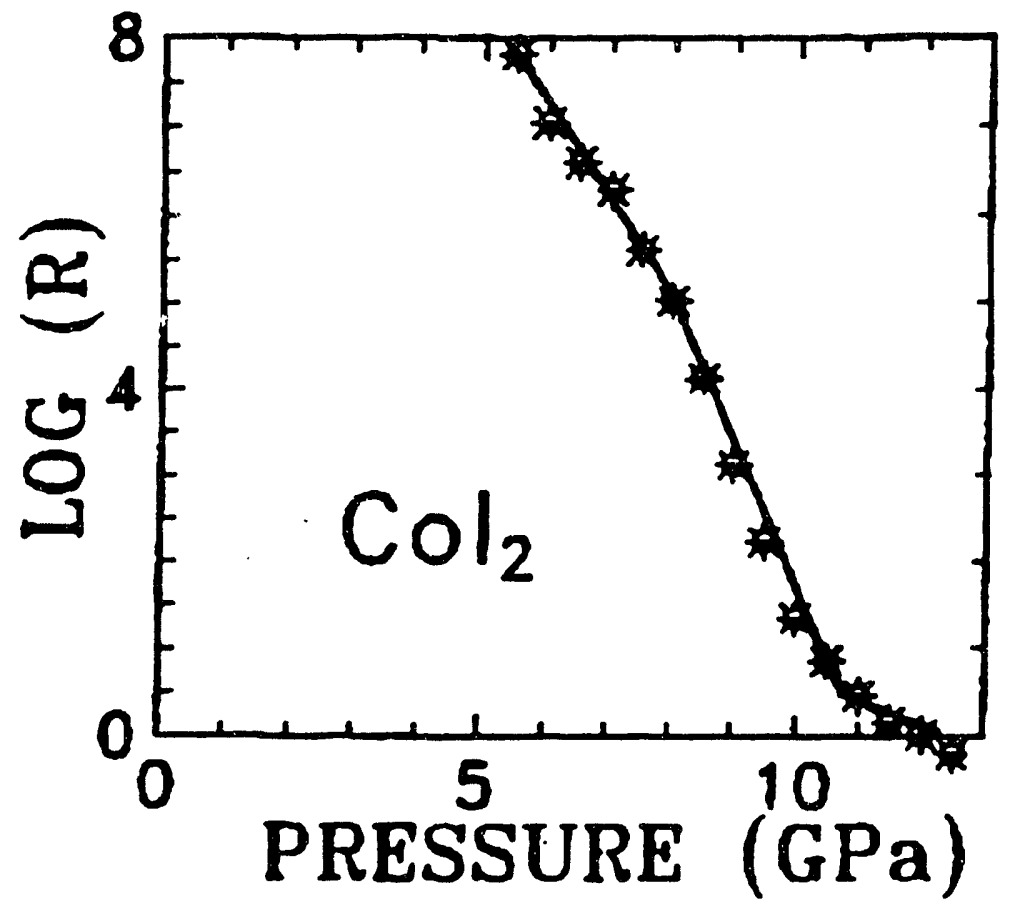

Fig. 6.5 


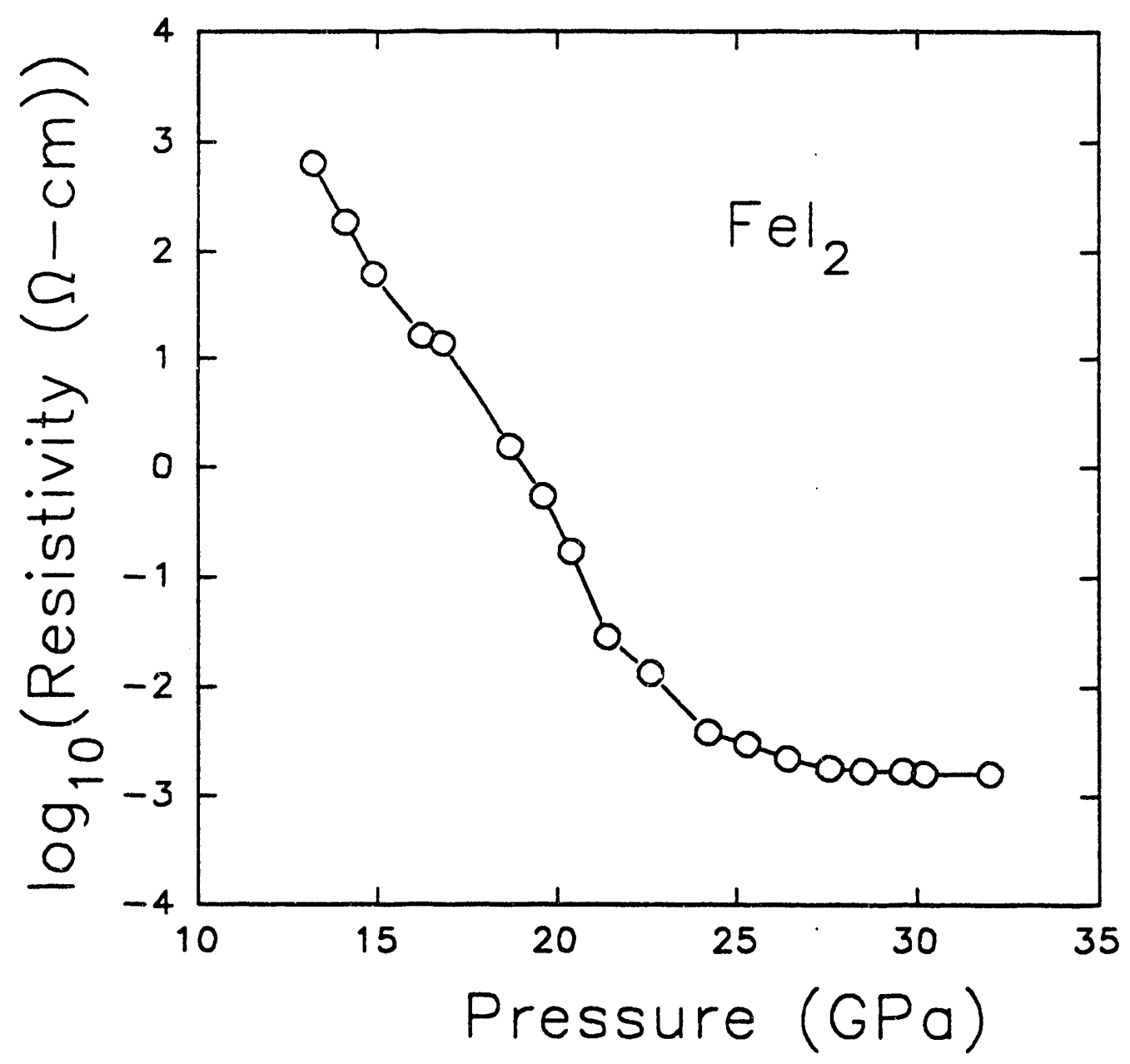

Fig. 6.6 


\section{Chapter VII: Absorption Measurements and Analysis}

To determine whether bandgap closure is causing metallization in the TMIs, we performed optical-absorption measurements. If the minimum energy gap is a dipole allowed transition, the fundamental absorption edge can easily be measured. Normal type Ia diamonds have a two phonon absorption band between $0.2 \mathrm{eV}$ and $0.3 \mathrm{eV}$ and have absorption from nitrogen impurities at lower energies. Therefore, we cannot follow the pressure dependence of any absorption edge to the point of closure. However, if the edge can be followed for a large range of pressures, it is possible to extrapolate its pressure dependence to the gap-closure pressure. This is a reasonable approach as long as structural phase transitions do not occur before the gap closure. Agreement between the extrapolated metallization pressure from the absorption measurements and the metallization pressure determined by resistivity measurements would be a strong indication that closure of the measured gap is the cause of the metallization. Furthermore, if the character of the minimum gap at ambient pressures were known, we could determine conclusively which gap is the cause of the metallization.

\section{A: Absorption Data of $\mathrm{NiI}_{2}$}

Spectra showing the absorbance of $\mathrm{NiI}_{2}$ at various pressures for light incident parallel to the c-axis are illustrated in Fig. 7.1. The spectra have been displaced vertically by one unit of the ordinate scale for clarity. The atmospheric-pressure spectrum contains two important features: a steeply rising absorption edge at $1.2 \mathrm{eV}$ and a small broad peak centered at approximately $0.9 \mathrm{eV}$. It has been deduced from a wealth of experimental data $[9,23,24,25]$ that this edge is associated with the charge-transfer transition. The lower energy peaks, which are designated by arrows, have been ascribed to the ${ }^{3} A_{28} \rightarrow{ }^{3} T_{28}$ transitions within the crystal-field split $\mathrm{Ni}^{2+} 3 d^{8}$ states $[24,25]$. Since these intracenter transitions are forbidden by parity conservation, these peaks are observable because of 
phonon participation [43]. The pressure dependence of these peaks is treated in the appendix.

Under increasing pressure the charge-transfer gap $\left(E_{\text {cт宀 }}\right)$ shifts to lower photon energies. To determine the energy of $E_{\text {CTG }}$ from our spectra we have simply extrapolated the absorption edge to the background absorption level as shown schematically in Fig. 7.1. This procedure is sufficient to determine the pressure dependence of $E_{\text {CTG }}$ since the absorption edge shifts uniformly with pressure. The resultant pressure dependence of $E_{\text {cTo }}$ is shown (closed circles) in Fig. 7.2. By fitting the data points to a straight line we find that $E_{\mathrm{cTG}}$ decreases with pressure at the rate of $-62 \mathrm{meV} / \mathrm{GPa}$ and extrapolates to zero at $21 \mathrm{GPa}$.

To investigate the position of the absorption edge at higher pressures, we also performed absorption measurements using a Fourier-transform infrared spectrometer, allowing measurements down to $0.3 \mathrm{eV}$. Three spectra, taken at $8 \mathrm{~K}$, show the absorption between 0.32 and $0.54 \mathrm{eV}$ at pressures of 12.8, 16.2, and 19.2 GPa (Fig. 7.3). They are not displaced vertically from each other. The $12.8 \mathrm{GPa}$ spectrum has a well defined absorption edge at about $0.53 \mathrm{eV}$. This data point is included in Fig. 7.2 and lies directly on the extrapolated line. If the edge continues to decrease linearly with pressure, the 16.2 GPa run should exhibit an edge at $-0.35 \mathrm{eV}$. Even if this edge is slightly lower in energy than predicted, one should be able to see at least the high-energy end of the absorption edge in the spectra in Fig. 7.3. Contrary to expectation this feature is absent and the 19.2 GPa spectrum shows a $1 / E$ dependence characteristic of free-carrier absorption, indicating that the sample is already metallic. Strangely, around $0.53 \mathrm{eV}$ both the $16.2 \mathrm{GPa}$ and the 19.2 GPa spectra actually have a lower absorbance than the 12.8 $\mathrm{GPa}$ run. If the metallization is caused by the precipitous closure of another lower energy gap, the absorption edge that we are following should still be present. Hence an increase in the absorption should occur around $0.5 \mathrm{eV}$. Most experimental artifacts, such as an increased reflectivity of the sample or a decrease in the gasket hole, lead to an increase in 
the observed absorbance and cannot account for the anomalous behavior. One plausible explanation is that the strength of the original absorption edge is drastically changed by the IM transition. These higher-pressure absorption measurements lead us to believe that the sample becomes metallic at a pressure between 16.2 and $19.2 \mathrm{GPa}$, which is consistent with the electrical resistivity results. The metallization pressure is therefore lower than suggested by the extrapolation of the charge-transfer gap.

\section{B: Absorption Data of $\mathrm{CoI}_{2}$ and $\mathrm{FeI}_{2}$}

The absorption spectra for $\mathrm{CoI}_{2}$ and $\mathrm{FeI}_{2}$ are shown in Figs.7.4 and 7.5. In $\mathrm{CoI}_{2}$ there is an absorption edge that begins at about $1.4 \mathrm{eV}$ at ambient pressures and decreases with increasing pressure. The peaks designated by arrows are assigned to an intra-3d transition within the $\mathrm{Co}^{2+}$ ion. The pressure dependence of this peak will be treated in the appendix. The absorption edge is determined in the same way as in the case of $\mathrm{NiI}_{2}$. Its pressure dependence is plotted in Fig. 7.6. A least-squares linear fit gives a gap-closure rate of $-82 \mathrm{meV} / \mathrm{GPa}$ and an extrapolated closure pressure of $17 \mathrm{GPa}$. This pressure is now significantly greater than the metallization pressure (10 GPa) determined from the resistivity results. The $10.1 \mathrm{GPa}$ absorption spectrum provides us with a hint of the reason for the discrepancy. That spectrum shows that the sample is now strongly absorbing (to within the amount of stray light) down to $0.5 \mathrm{eV}$ with no sign of an absorption edge even though, according to the extrapolation, the gap should be $0.6 \mathrm{eV}$. This is similar to the behavior in $\mathrm{NiI}_{2}$ but even more pronounced. Again, if the gap was at 0.3 or $0.4 \mathrm{eV}$ we should observe at least the high tail end of the absorption edge. The $10.1 \mathrm{GPa}$ spectrum suggests instead that the sample is already metallic at that pressure, in agreement with the resistivity measurement.

In $\mathrm{FeI}_{2}$ the behavior of $E_{\text {CTG }}$ is also quite similar to the other two iodides. The gap closure rate and the extrapolated gap closure pressure are $-90 \mathrm{meV} / \mathrm{GPa}$ and $22 \mathrm{GPa}$ respectively (Fig 7.6). However, unlike $\mathrm{NiI}_{2}$ or $\mathrm{CoI}_{2}$ we did not see any indication of a 
metallic phase in the absorption spectra up to $16.9 \mathrm{GPa}$. This is also consistent with the electrical resistivity results which suggest that $\mathrm{FeI}_{2}$ metallizes around $23 \mathrm{GPa}$. Out of the three TMIs that we have studied, $\mathrm{FeI}_{2}$ seems to be the only one where the extrapolated gap closure and metallization occur at the same pressure. 


\section{Figure Captions for Chapter VII}

Fig. 7.1 $\mathrm{NiI}_{2}$ near-IR absorbance spectra as a function of pressure. The spectra are displaced vertically from each other by 1 unit. The straight lines represent the absorption edge. The remaining peaks, which are marked with arrows, are identified with intra-3d transitions. The value of the charge-transfer gap is determined by the intercept of the line with the horizontal axis. All spectra were recorded at $300 \mathrm{~K}$.

Fig. 7.2 $\mathrm{NiI}_{2}$ charge-transfer energy gap and carrier-activation energy vs. pressure. The straight line is a least-squares fit to the data; it extrapolates to a zero gap at $21 \mathrm{GPa}$.

Fig. 7.3 Absorbance of $\mathrm{NiI}_{2}$ between 0.3 and $0.55 \mathrm{eV}$ recorded at $8 \mathrm{~K}$ for three different pressures. The $12.8 \mathrm{GPa}$ spectra shows an absorption edge at about $0.53 \mathrm{eV}$ while the 16.2 and 19.2 GPa runs show evidence of free-carrier absorption. Notice that the graphs are not displaced vertically from each other as in Fig. 7.1. Undulations in the spectra are Fabey-Perot-type interference fringes caused by the diamond anvils.

Fig. 7.4 $\mathrm{CoI}_{2}$ near-IR absorbance spectra as a function of pressure. The spectra are displaced vertically from each other by 1 unit. The spectra are decomposed into an absorption edge and the intra $3 d$ transitions (indicated by arrows) as in Fig. 7.1. All spectra were recorded at $300 \mathrm{~K}$.

Fig. 7.5 $\mathrm{Fel}_{2}$ near-IR absorbance spectra as a function of pressure. The spectra are displaced vertically from each other by 1 unit. The analysis of the spectra is the same as in Figs. 7.1 and 7.4. All spectra were recorded at $300 \mathrm{~K}$. 
Fig. 7.6 Charge-transfer energy gap as a function of pressure for $\mathrm{CoI}_{2}$ and $\mathrm{FeI}_{2}$. The straight lines are least-squares fits to the data. They extrapolate to a gap closure pressures of 17 and $22 \mathrm{GPa}$ for $\mathrm{CoI}_{2}$ and $\mathrm{FeI}_{2}$ respectively. 


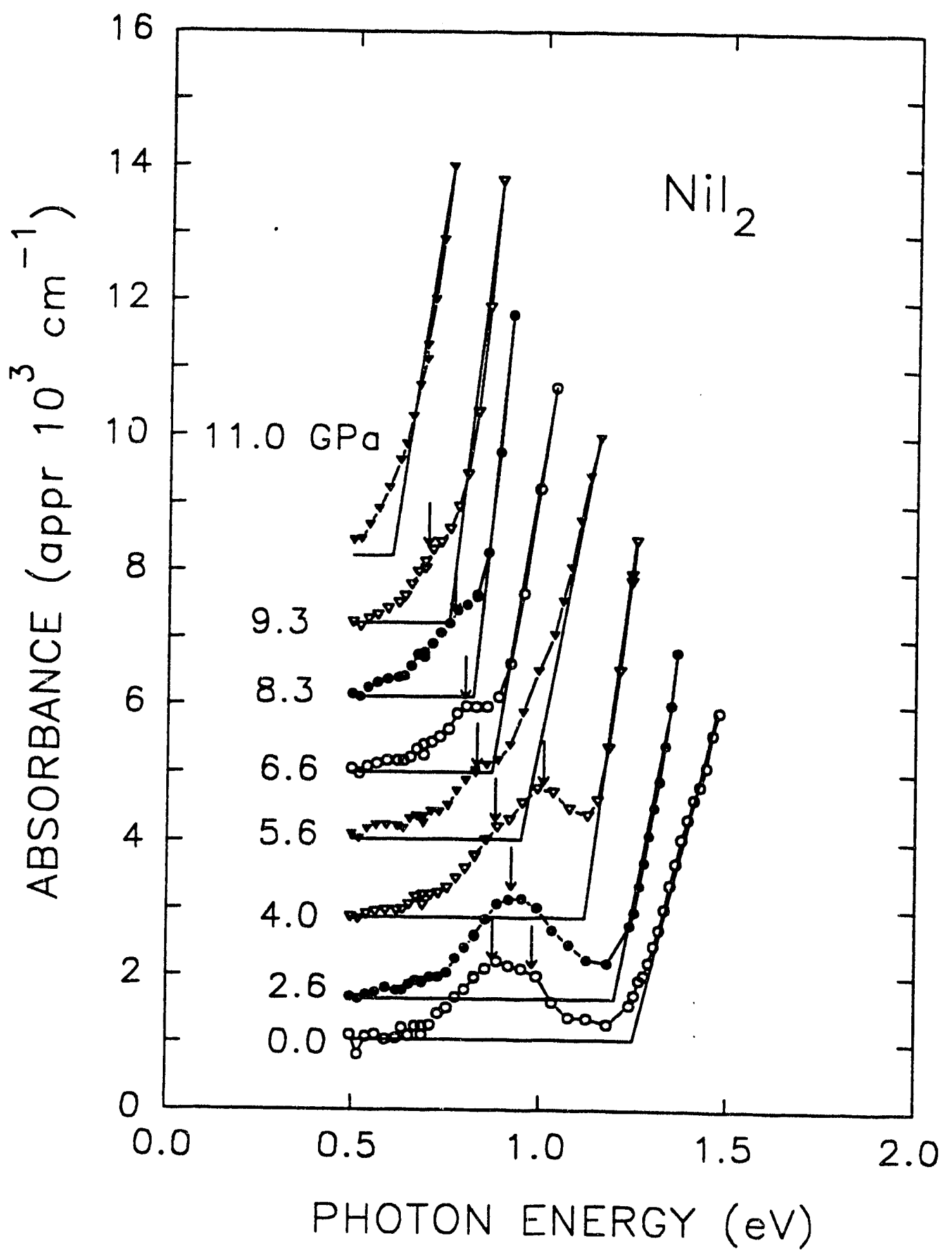

Fig. 7.1 


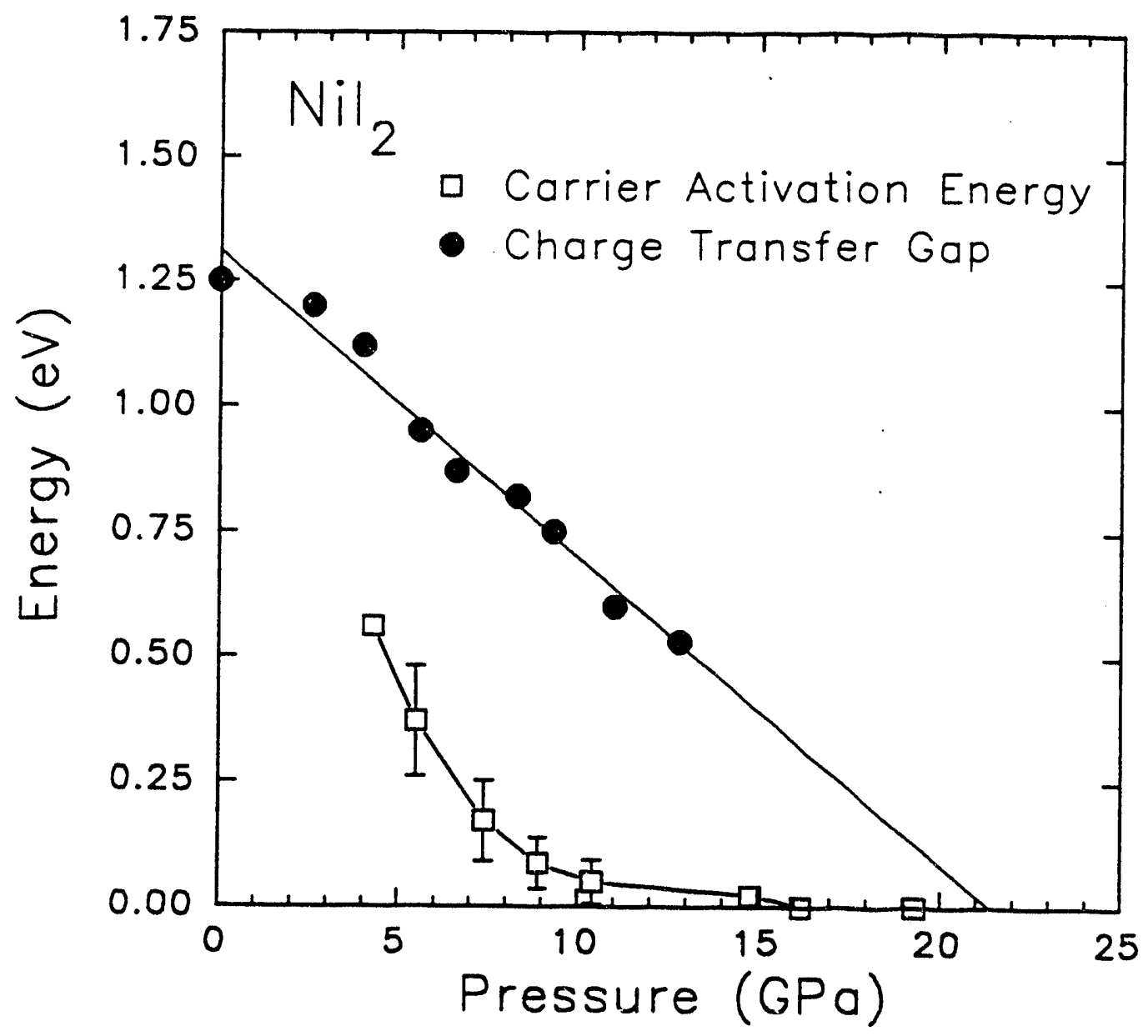

Fig. 7.2 


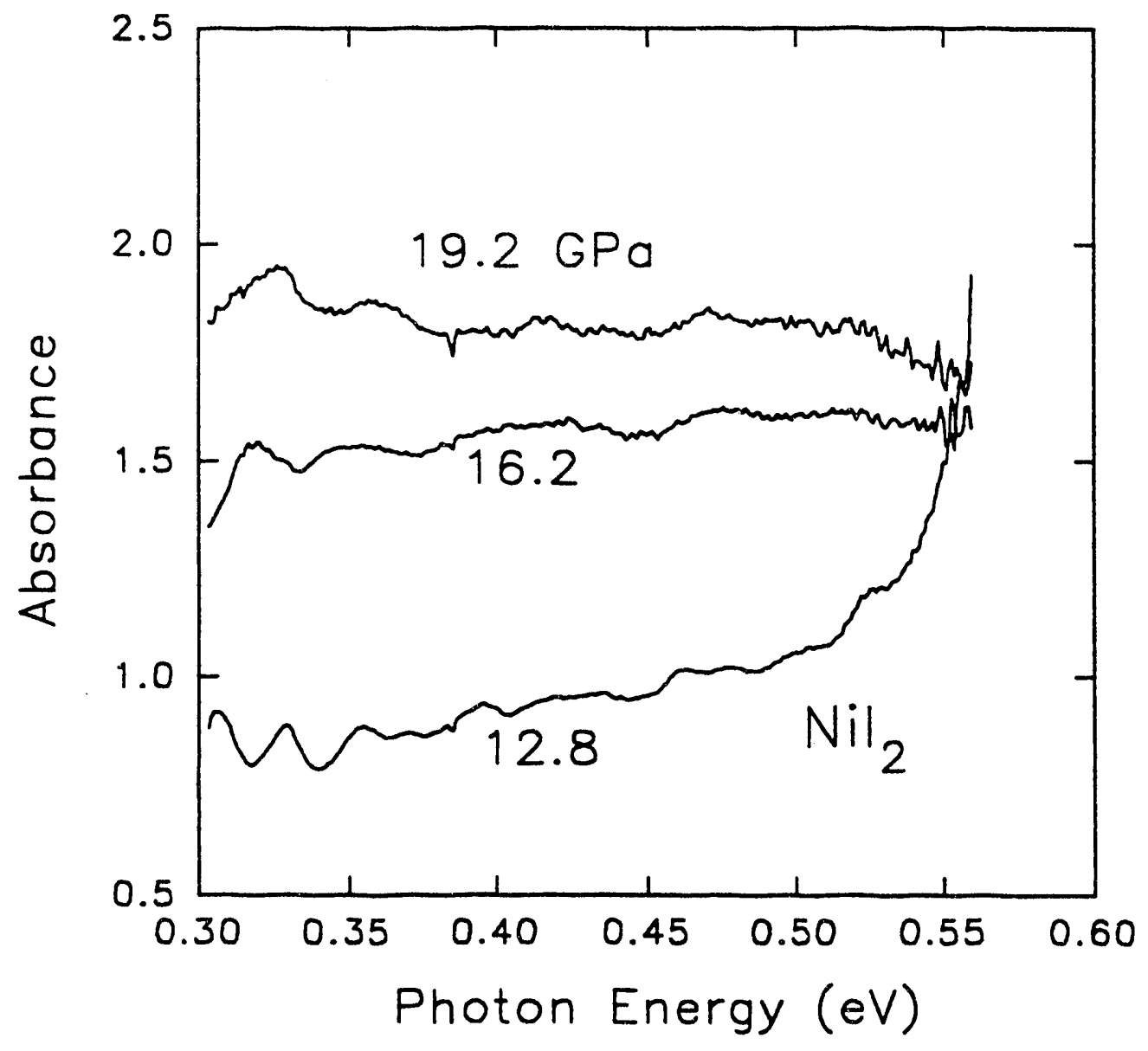

Fig. 7.3 


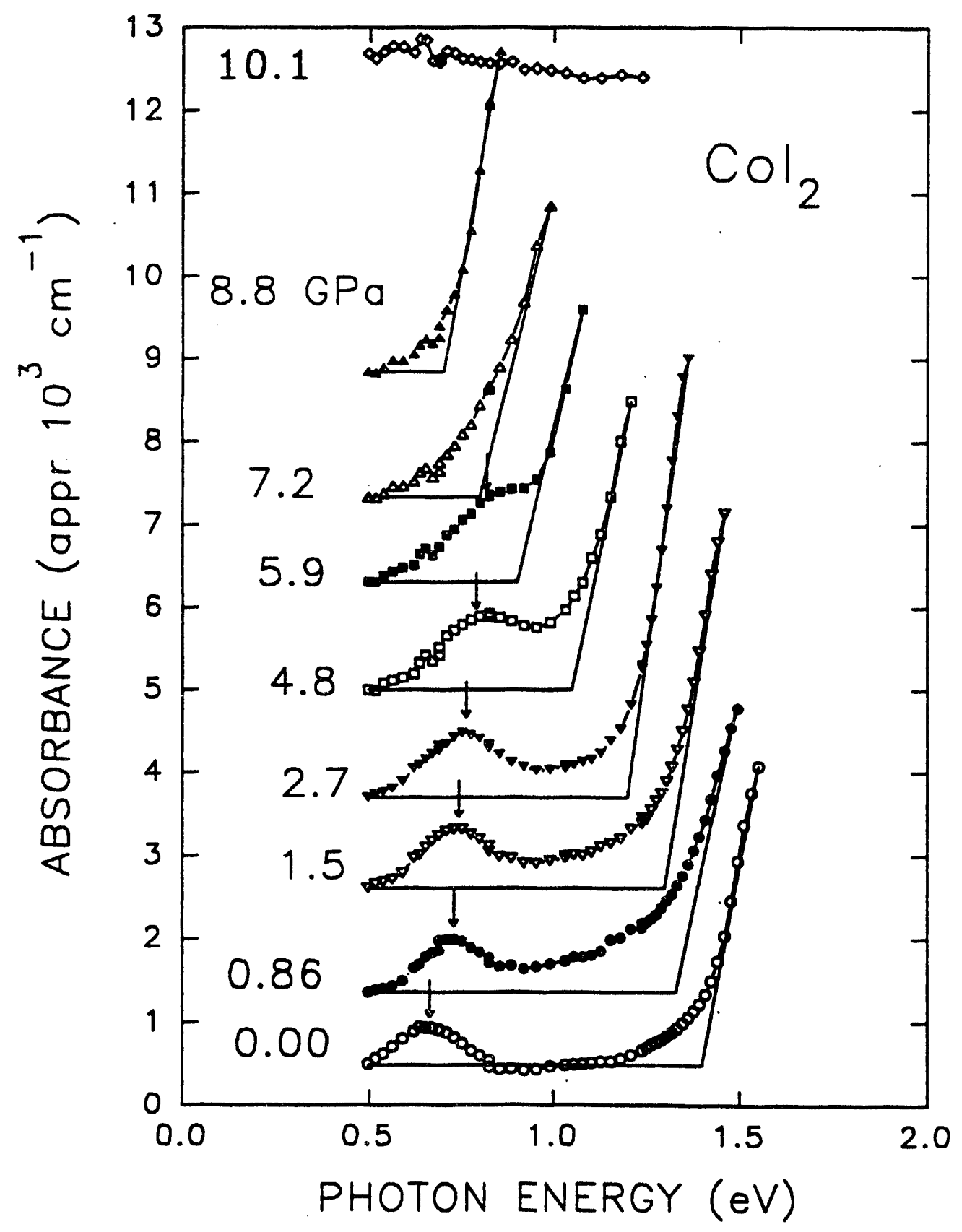

Fig. 7.4 


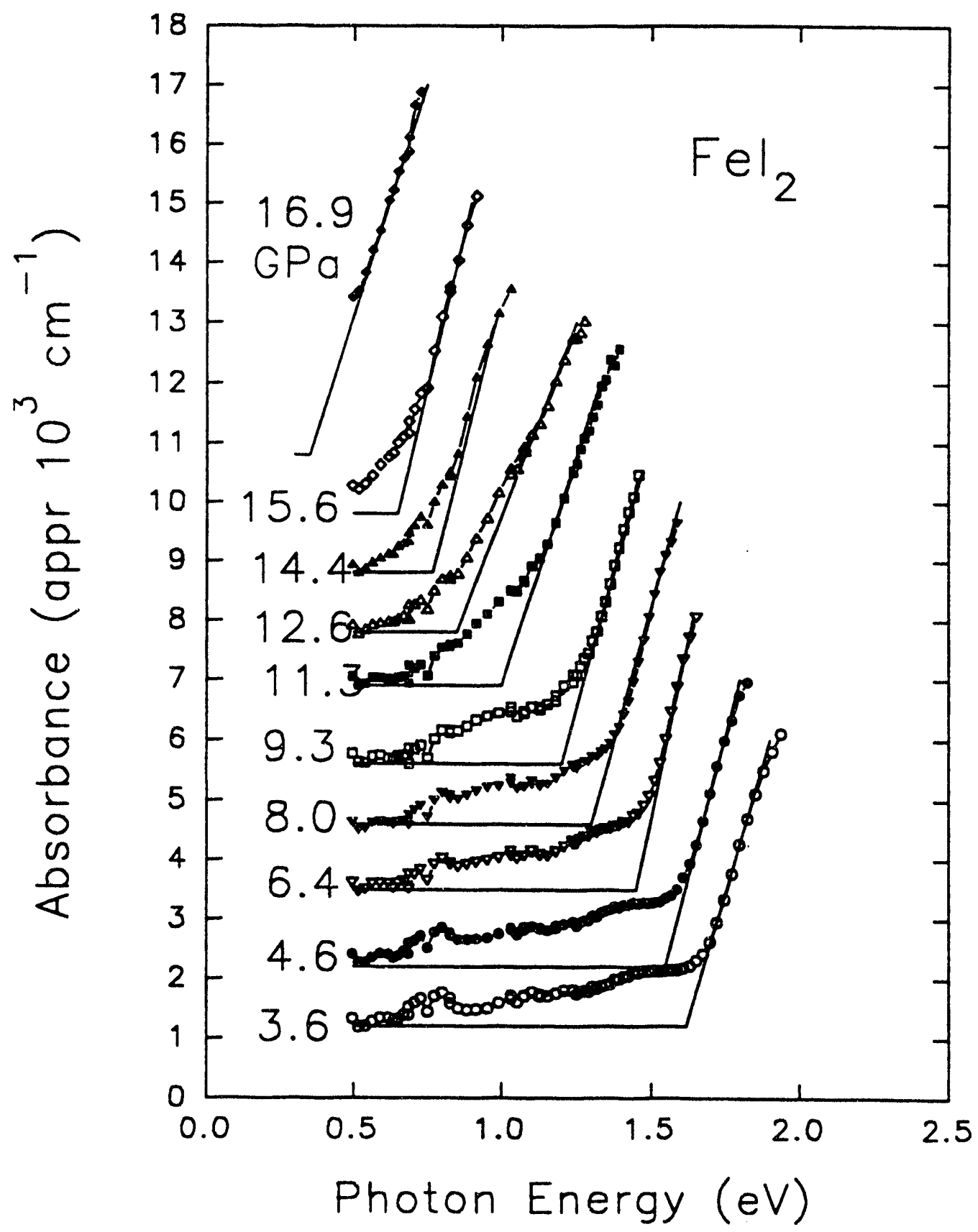

Fig. 7.5 


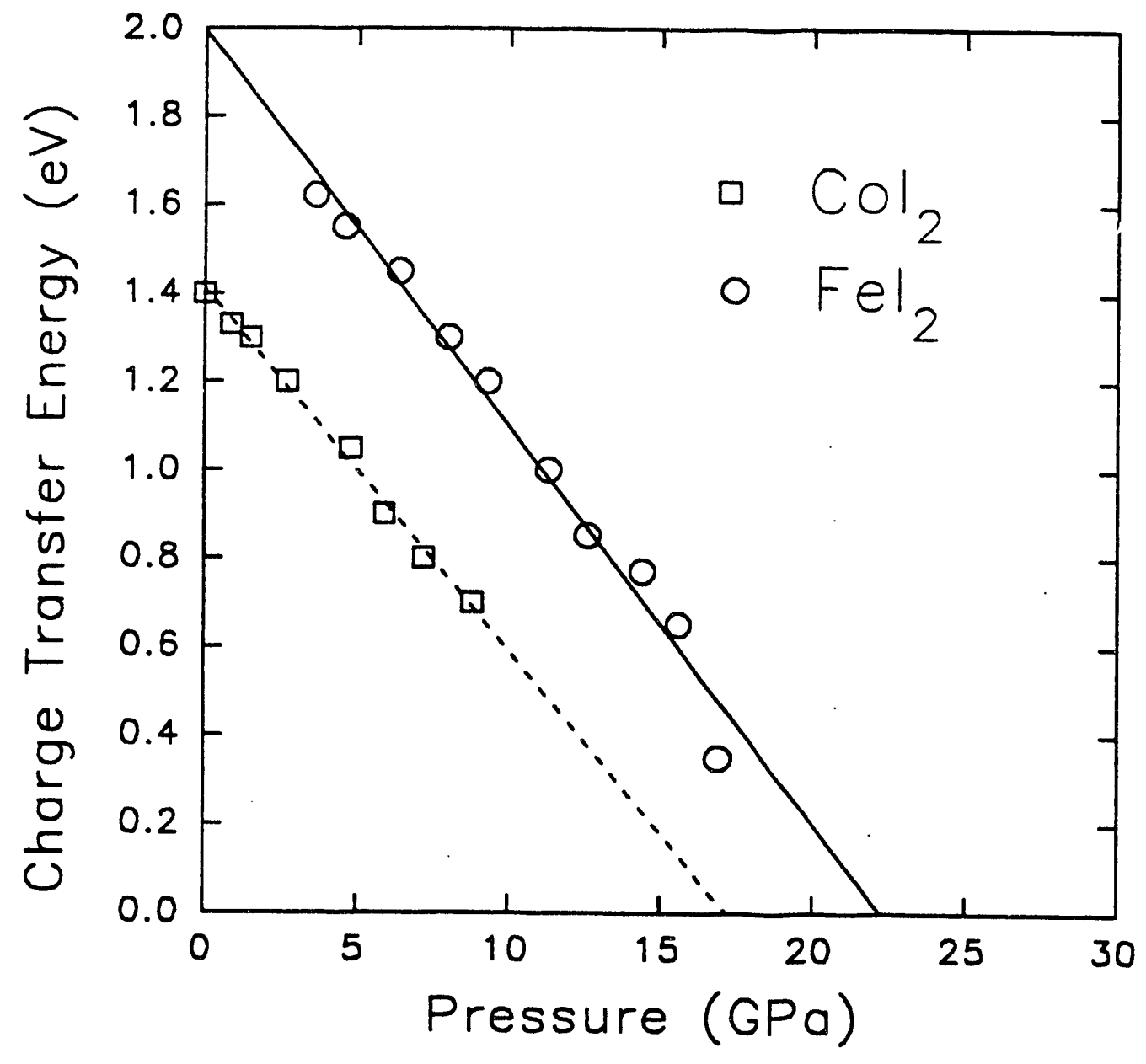

Fig. 7.6 


\section{Chapter VIII: Discussion}

The experimental data indicate that the transition-metal iodides undergo both $\mathbf{I M}$ and magnetic phase transition(s) under pressure. In this chapter we discuss the relationship between the observed magnetic and $\mathrm{IM}$ transitions. We also discuss the relationship between the resistivity and absorption measurements, in particular we argue that our data conclusively show metallization in the TMIs are induced by charge-transfer gap closure. We present evidence which points to a first-order phase transition in $\mathrm{NiI}_{2}$ and $\mathrm{CoI}_{2}$. Lastly we compare our experimental findings with the calculated phase diagrams of these materials.

\section{A: Origin of the IM Transitions and Its Relation to the Magnetic Transitions in the TMIs}

Because both the magnetic and the $\mathrm{MM}$ transitions of $\mathrm{NiI}_{2}$ occur within a narrow pressure range, the evidence strongly suggests that the two are related. The association is equally apparent in $\mathrm{CoI}_{2}$ although details are complicated by the coexistence of two phases after metallization. Any model that explains these results must inherently include the interrelation between the two kinds of transitions. Furthermore, the model must involve electronic changes orly since there is no change in crystal structure. The resistivity data imply that a transport gap continuously closes as a function of pressure, and the model calculations outlined in Chapter III assume that this gap is the charge-transfer gap. Although photoemission results suggest that these TMIs are charge-transfer insulators at ambient pressure, the effect of pressure on all its energy gaps is unknown. Therefore, we take a broader approach and consider the possible pressu:e-induced closing of three different gaps in the discussion below. These three scenarios are shown schematically in Fig. 8.1.

The $\mathrm{Ni}^{2+} 4 s$ band is the lowest energy extended conduction band. Under pressure it may widen so that its lower edge moves down and crosses the lower Hubbard band (Fig. 
8.1a). If a transfer of electrons from the $3 d^{8}$ shell to the $4 s$ band $\left(4 s^{0} 3 d^{8} \rightarrow 4 s^{1} 3 d^{7}\right)$ results, the magnetic moment of the $\mathrm{Ni}^{2+}$ would increase. Sirce the exchange coupling $J$ is proportional to the total spin $S$, an increase in $S$ is not likely to suppress the antiferromagnetic state. However, the $4 s$ conduction electrons can also screen the magnetic moments [2]. There are no reliable experiments or calculations that provide the relative location of the $4 s$ and the upper Hubbard band in $\mathrm{NiI}_{2}$. Only inverse photoemission can provide such information. Pseudopotential calculations utilizing the local-density approximation (LDA) are very reliable in determining ground-state energies but invariably underestimate the excited state energies. This scenario, of course, assumes that under pressure the $\mathrm{Ni}^{2+} 3 d$ states move to the top of the valence band.

The second possibility is the closure of the Mott-Hubbard gap, $E_{\mathrm{MHG}}$ (Fig. 8.1b). Excitations across the Mott gap are written as $3 d^{h} 3 d^{h} \rightarrow 3 d^{h-1} 3 d^{h+1}$. Under increasing pressure, lattice constants decrease and the overlap between the $d$ wavefunctions of adjacent $\mathrm{Ni}^{2+}$ ions increases, possibly widening the bandwidths. If the $d$ bands widen enough so that the two bands overlap, metallization results. This is the classic Mott transition from localized to delocalized states. The disappearance of the magnetic moments may be accounted for by screening from the conduction electrons or decrease in exchange energy resulting from delocalization.

Lastly we have the possibility that the charge-transfer gap $\left(E_{\text {crG }}\right)$, the energy gap between the I- $5 p$ band and the upper Hubbard band, closes (Fig. 8.1c). The chargetransfer energy $\Delta$ is essentially the energy required to move an electron from the anion $p$ band onto the transition-metal $d$ shell and is schematically written in the literature as $\Delta=$ $3 a^{\text {h }} \rightarrow 3 d^{\text {h+1 }} L$ where $L$ represents a ligand hole. $\Delta$ increases strongly with increasing anion electron affinity ard studies of transition-metal halides where the halogen is varied from fluorine to iodine confirm this $[24,25]$. Again $E_{\text {cto }}$ can close under pressure by an increasing anion $5 p$ bandwidth, $B_{\mathrm{p}}$. Once overlap occurs, electrons spill over into the upper Hubbard band decreasing the magnetic moment and suppressing the 
antiferromagnetic exchange by filling the $3 d$ shell. The holes created in the $5 p$ band by the transfer to the $d$ bands are responsible for the conduction. At the point where $E_{\text {ст⿻ }}$ disappears there are also holes and electrons in the upper Hubbard band but they are much heavier than the $5 p$ holes and are expected to contribute comparatively less to the conductivity.

As previously pointed out in Chapter II a study of the Ni dihalides shows that for $\mathrm{NiI}_{2}, U \sim 4.5 \mathrm{eV}$ while $\Delta \sim 1.5 \mathrm{eV}$ suggesting that $\mathrm{NiI}_{2}$ is a charge-transfer insulator[9]. The values obtained from photoemission most likely reflect the gaps $E_{\mathrm{MHG}}$ and $E_{\mathrm{cTG}}$ or their associated excitons instead. From the reflectivity study $\Delta$ is determined to be about $4.0 \mathrm{eV}$ [23]. From valence band photoemission data of $\mathrm{NiI}_{2}$ and band-structure calculations of isostructural $\mathrm{NiBr}_{2}$ one estimates that $B_{\mathrm{p}} \sim 5 \mathrm{eV}$ and $B_{\mathrm{U}} \sim 0.5 \mathrm{eV}[21,22]$ $B_{\mathrm{s}}$ is unknown but is probably smaller than thr $B_{\mathrm{p}}$ because the $4 s$ electrons are more tightly bound than the $5 p$ electrons. Because $B_{p}$ is already large, compression will increase it more rapidly than the other bandwidths. The values of $\Delta(4.0 \mathrm{eV}), B_{\mathrm{U}}(0.5 \mathrm{eV})$, and $B_{\mathrm{p}}$ (5 eV), when substituted into Eq. (2.1), give a value of $E_{\text {ctG }}$ of $-1.2 \mathrm{eV}$. There is a large uncertainty in this estimate because we are taking the difference of two larger numbers, both of which have uncertainties. From the resistivity data we have shown that $E_{t}$ extrapolates to a zero pressure value of about $1.1 \mathrm{eV}$ (Fig. 6.4), which agrees closely with the ambient pressure value of $E_{\text {стG }}$. These facts taken together makes closure of $E_{\text {cTG }}$ the most likely candidate for explaining the $\mathrm{IM}$ transition in $\mathrm{NiI}_{2}$. In the ZSA picture the charge-transfer materials can be either metals or insulators depending on the values of $\Delta$ and $B_{\mathrm{p}}$. In our work we have shown that pressure can increase $B_{\mathrm{p}}$ and induce this IM transition in the TMIs.

In theory the three possibilities can be differentiated by using a Hall effect measurement to determining the sign of the charge carriers. Cases (a) and (c) in Fig. 8.1 would have $n$ and $p$ type conduction respectively while case (b) should show a very weak Hall coefficient and very low mobility. In each case the large effective masses of the $d$ 
electrons effectively prohibits them from participating in the conductivity when compared to the highly mobile $4 s$ and $5 p$ electrons. In practice we found that we could not obtain a Hall signal because high-quality single crystals are necessary. We found that during the initial pressurization ( 0 to $0.5 \mathrm{GPa}$ ) the resistivity of $\mathrm{NiI}_{2}$ increased dramatically. Presumably dislocations at these pressures decrease the mobility. The signal-to-noise ratio of the Hall signal is proportional to carrier mobility so that any decrease in mobility makes a Hall measurement difficult. We have not been able to make a Hall effect measurement on $\mathrm{NiI}_{2}$ under pressure despite repeated attempts.

\section{B: Discussion of the Near-IR Absorption Data: \\ Metallization by Charge-Transfer Gap Closure}

We have seen in Chapter VII that near-IR absorption measurements in $\mathrm{NiI}_{2}$ show an edge that decreases linearly with increasing pressure and extrapolates to zero at 21 $\mathrm{GPa}$. This pressure is close, although higher, than the metallization pressure determined by the resistivity measurements. As discussed in Chapter II, the ambient pressure studies of the late-transition-metal halides suggest that they are charge-transfer insulators $[9,23$ 25]. These results all point to the possibility that closure of the charge-transfer gap is responsible for the $\mathrm{IM}$ transition in $\mathrm{NiI}_{2}$. In this section we will examine this idea further.

The pressure-induced shift of $E_{\text {CTG }}$ can mostly be attributed to a widening of the $5 p$ band. In Eq. (2.1) $B_{\mathrm{v}}$ and $B_{\mathrm{p}}$ are the bandwidth of the upper Hubbard band and the I$5 p$ band respectively. Pressure can presumably affect both $\Delta$ and the two bandwidths. While the pressure dependence of $\Delta$ is difficult to estimate, the pressure dependence of $B_{\mathrm{p}}$ can be easily calculated within the tight-binding approximation if the pressure-volume relation is known. If we assume that $B_{\mathrm{p}}$ is abcut $5 \mathrm{eV}$ at atmospheric pressure as oeduced from photoemission measurements and band-structure calculations [16,17], and $B_{\mathrm{p}}$ varies with the sample volume $V$ as $V^{-2 / 3}$ [44] then using the pressure-volume data (Fig. 4.5) we arrive at a value of $d\left(-B_{\mathrm{p}} / 2\right) / d P=-37 \mathrm{meV} / \mathrm{GPa}$. Since $B_{\mathrm{U}}$ is initially about $0.5 \mathrm{eV}$, a 
similar estimate of its pressure dependence yields a much smaller value, $\left(d\left(-B_{v} / 2\right) / d P=-4 \mathrm{meV} / \mathrm{GPa}\right)$. The sum of these two values is comparable to the experimental value of $d E_{\mathrm{CTG}} / d P=-62 \mathrm{meV} / \mathrm{GPa}$ for the case of $\mathrm{NiI}_{2}$. We conclude that the pressure-induced decrease in the charge-transfer gap is caused predominantly by the broadening of the iodine $5 p$ bands.

In Fig. 7.2, we compare the transport gap $E_{t}$ of $\mathrm{NiI}_{2}$ determined by the Arrhenius plots directly with $E_{\text {CTG }}$ as a function of pressure. Although the value $E_{l}$ does not follow $E_{\text {CTG }}, E_{t}$ always remains less than $E_{\text {CTG }}$ and both decrease monotonically. It is thus plausible that $E_{t}$ is determined by some levels within the charge-transfer gap. In our temperature-dependent measurements we observe only these levels because the temperature is low in comparison with $E_{\text {ctG }}$. The origin of these levels is not clear at this moment. They may be associated with dislocations or other defects created by the nonhydrostatic component of the applied stress. These levels may only be present in the electrical measurements because of the powder pressure medium. $E_{t}$ cannot be a measurement of $U$ since the value of $U$ determined from photoemission at atmospheric pressure $(\sim 5 \mathrm{eV})$ is much larger than the value of $E_{t}$ extrapolated to atmospheric pressure from Fig. 7.2.

As argued in Chapter VI, the resistivity measurements place the metallization pressure in $\mathrm{NiI}_{2}$ at $17 \pm 1 \mathrm{GPa}$. However, $E_{\text {сто }}$ determined by the absorption measurements extrapolates to zero at $21 \mathrm{GPa}$. Perhaps this difference in the metallization pressures determined by resistivity and absorption measurements may be attributed to the different pressure media used. In the case of the resistivity measurements, the inherent quasi-hydrostaticity of the medium coupled to the anisotropy of the TMI crystal str cture may change the observed transition pressure. Nevertheless, the decrease in $E_{\text {cTo }}$ and $E_{t}$ roughly parallel each other over a large pressure range. In addition it should be noted that the closure of $E_{\text {CTg }}$ will automatically also force the closure of any states within the charge-transfer gap. These considerations strongly suggest that the metallization in $\mathrm{NiI}_{2}$ 
is fundamentally driven by the closure of the charge-transfer energy gap. Closing of the Mott-Hubbard gap, $U$, is a less likely candidate since its value is about $4.5 \mathrm{eV}$ at ambient pressures.

It should be noted that the edge in our absorption measurements may actually correspond to the lower energy edge of the charge-transfer exciton peak, and not the fundamental $p \rightarrow d$ absorption peak. We do not know the effective mass of the anion $5 p$ holes and thus cannot estimate the exciton binding energy even if we do assume that the $3 d$ electrons are completely dispersionless. The optical and $\mathrm{UV}$ reflectivity data on $\mathrm{NiI}_{2}$ show the exciton peak to be located at $\sim 2 \mathrm{eV}$ while the charge-transfer continuum is centered at $4-5 \mathrm{eV}$, but do not clearly indicate where the edge of the continuum is relative to the charge-transfer exciton. Because of the uncertainty in the $p$ bandwidth, the exciton binding energy is difficult to estimate.

The absorption data of $\mathrm{CoI}_{2}$ and $\mathrm{FeI}_{2}$ coupled with their resistivity data as a function of pressure further reinforce the conclusion that metallization in this family of compounds is caused by charge-transfer gap closure. $\mathrm{CoI}_{2}$ metallizes at $-9-10 \mathrm{GPa}$ and its $E_{\text {СтO }}$ extrapolates to zero at $17 \mathrm{GPa}$ while in $\mathrm{FeI}_{2}$ both resistivity and extrapolation of $E_{\text {CTG }}$ show metallization at 22-23 GPa. The trend and agreement between the two types of measurements is too close to be coincidental.

\section{C: Evidence for a First-Order Insulator-Metal Transition}

Within the ZSA framework, the magnitude of the charge-transfer gap is sufficient to separate the metallic from the insulating phases. It cannot, however, describe the thermodynamics of the $\mathrm{IM}$ transition because other interactions can become important when $E_{\text {CTG }}$ is of the order of elementary excitations of the solid such as phonons. All relevant interactions will ultimately determine the pilase boundaries in this sensitive region. In this section we discuss the evidence that shows that the $\mathbb{I M}$ transition may be first-order 
in $\mathrm{NiI}_{2}$ and $\mathrm{CoI}_{2}$ and suggest that the transition is caused by spontaneous formation of charge-transfer excitons, as the model calculations described in Chapter IV predict.

In the $\mathrm{NiI}_{2}$ measurements three experimental findings suggest a first-order transition. As previously mentioned the resistivity-pressure curves in both Fig. 6.1 and 6.2 show a conspicuous knee that is not predicted by a continuous bandgap closure but rather seems to indicate a discontinuity in the resistance that is smoothed over by the pressure inhomogeneity. The extrapolation of $E_{\mathrm{crG}}$ suggests a metallization pressure $(21 \mathrm{GPa})$ that is higher than that determined by resistivity. Finally the higher pressure absorption measurements show evidence of free-carrier absorption and no edge at a pressure of 16.2 $\mathrm{GPa}$ well before is $E_{\mathrm{CTG}}$ extrapolated to close. These three points are naturally explained by a first-order, discontinuous gap closure at about $16 \mathrm{GPa}$. One might alternatively interpret the evidence as caused by the rapid closing of another energy gap that is originally above $E_{\mathrm{CTG}}$ but overtakes it at $\sim 16 \mathrm{GPa}$. This proposal does not explain why the charge-transfer edge is not visible on top of the free-carrier absorption in the $16.2 \mathrm{GPa}$ spectrum in Fig. 7.3. The disappearance of the absorption edge in the $16.2 \mathrm{GPa}$ spectrum is strong evidence that the charge-transfer gap collapses to zero discontinuously at $\sim 16$ GPa.

In $\mathrm{CoI}_{2}$ the discontinuous closure is also evident and seems to be even larger. There is a large knee in the resistance measurentent (Fig. 6.5) and the discrepancy between the extrapolated gap closure pressure (17 GPa) and the resistance metallization (10 GPa) is much larger. The absorption spectrum at $10.1 \mathrm{GPa}$ shows a strong and flat absorption down to $0.5 \mathrm{eV}$ whereas if the gap closes linearly it should show an edge at $0.6 \mathrm{eV}$ (Fig 7.4). This is evidence that the $\mathrm{IM}$ transicion is more strongly first-order in $\mathrm{CoI}_{2}$ than in $\mathrm{Nil}_{2}$ with $E_{\text {ctG }}$ closing from 0.6 to $0 \mathrm{eV}$ at $\sim 10 \mathrm{GPa}$. The Mössbauer data for $\mathrm{CoI}_{2}$ (Fig. 4.4) suggests that between 9-13 GPa two phases, one antiferromagnetic and one nonmagnetic, coexist. In addition there is a discontinuous isomer shift implying a change in bonding associated with the phase transitions. We speculate that the ongoing $x$-ray 
measurements for $\mathrm{CoI}_{2}$ will show a slight lattice discontinuity. In contrast the $\mathrm{FeI}_{2}$ results do not indicate that the transition is first-order even though the physics of the transition should be similar to that of $\mathrm{NiI}_{2}$ and $\mathrm{CoI}_{2}$.

Considerations by Mott and the model calculations. of Giesekus et. al. and Freericks et. al. (described in Chapter IV) both predict that band-overlap transitions in these materials should be first-order due to excitonic effects [2,26]. Although these models are not quantitative, they do contain the relevant physical concepts. Our work provides direct evidence that the assumptions and approximations made in these models are essentially correct. More specifically in $\mathrm{NiI}_{2}$ the metallization at $17 \mathrm{GPa}$, as evidenced by the transport measurements, suggests that there is a region between 17 and $19 \mathrm{GPa}$ that is both antiferromagnetic and metallic. The metallization seems to coincide with the onset of a decrease in the saturation hyperfine magnetic field at $\sim 16 \mathrm{GPa}$ before it completely disappears at $19 \mathrm{GPa}$. It is possible that there art separate magnetic and $\mathrm{IM}$ transitions. It has been suggested in the work of Giesekus et. al.[30] that for some parameters there is a first-order $\mathbf{I M}$ transition followed by a secord-order magnetic to non-magnetic transition.

If the $\mathrm{IM}$ transition is induced by the formation of excitons, the excitons must exist in an electron-hole liquid phase to exhibit metallic conductivity. The energy of the observed edge just before metallization (size of the gap discontinuity) should be the cohesive energy per exciton of the electron-hole liquid. We remind the reader that the edge itself is the free exciton absorption edge. Because there may be volume changes associated with this transition, other energies have to be carefully considered before we claim that the gap discontinuity quantitatively' reflects the previously mentioned cohesive energy.

The coexistence of two phases in $\mathrm{CoI}_{2}$ is rather puzzling. If the transition is firstorder there should be a discontinuity in the lattice constants. In $\mathrm{NiI}_{2}$ it is probably below the resolution of the $\mathrm{x}$-ray measurements. In $\mathrm{CoI}_{2}$ we expect it to be larger. One possible reason why two phases seem to coexist is that the Mössbauer measurements were taken 
with an unusual amount of pressure inhomogeneity. Pressure inhomogeneity is a serious problem in resistivity measurements where $\mathrm{CaSO}_{4}$ powder is used as the pressure medium. Since Mössbauer measurements typically use $\mathrm{Ar}$ as the pressure medium, pressure inhomogenieity should be much smaller than the range of $4 \mathrm{GPa}$ over which the two phases were found to coexist. The second possibility is that stresses within the crystal during the transformation widen the observed transition pressure by impeding a complete transition into the high-pressure phase. This effect has recently been theoretically studied [39a] and is expected to occur even in a completely hydrostatic medium. 


\section{Figure Captions for Chapter VIII}

Fig. 8.1 Density-of-states diagrams of the three possible scenarios for metallization by band gap closure in the transition-metal iodides. a) Closure of the gap between the $4 s$ band and lower Hubbard band. b) Closure of the Mott-Hubbard gap. c) Closure of the charge-transfer gap. 


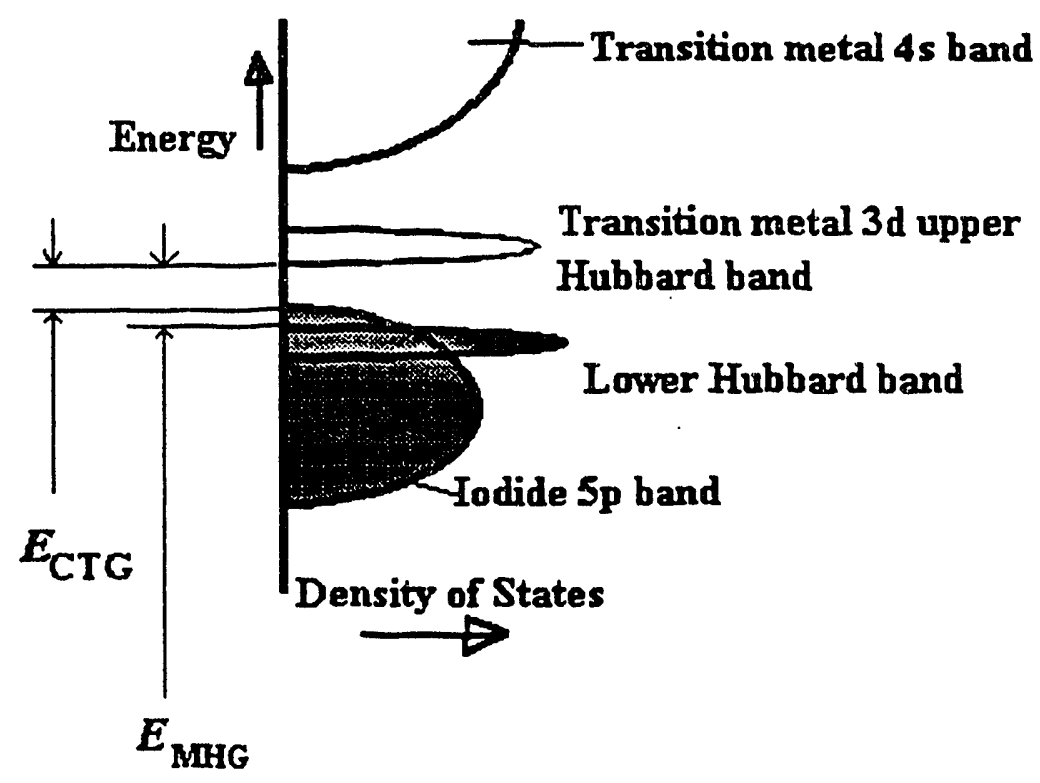

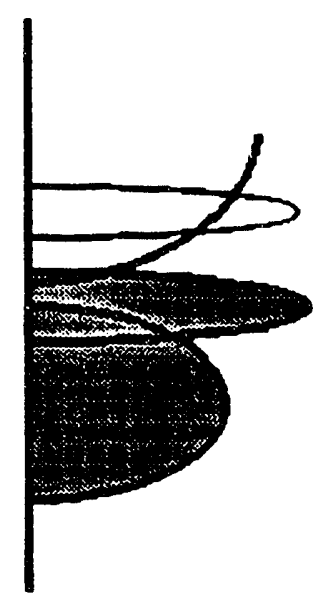

a: gap closure between $4 s$ and lower Hubbard band

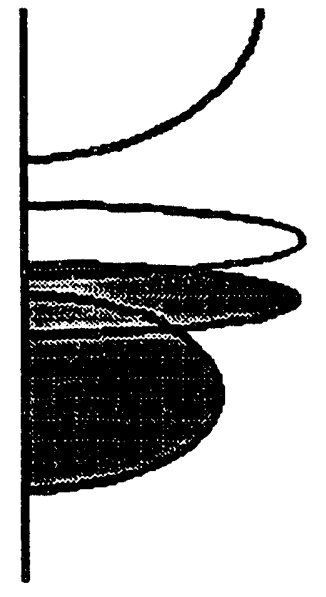

b: Mott Hubbard gap closure

Fig. 8.1

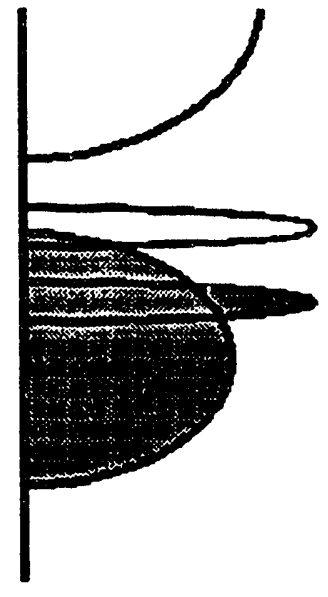

c: charge-transfer gap closure 


\section{Chapter IX: Conclusion and Future Efforts}

We have shown with resistivity and near-IR absorption measurements that the transition-metal halides $\mathrm{NiI}_{2}, \mathrm{CoI}_{2}$, and $\mathrm{FeI}_{2}$ metallize under pressure by closure of the charge-transfer energy gap at pressures of 17,10 , and $23 \mathrm{GPa}$ respectively. The closure of the charge-transfer energy gap occurs at a pressure that is close to the antiferromagnetic-diamagnetic transitions reported in $\mathrm{NiI}_{2}(19 \mathrm{GPa})$ and $\mathrm{CoI}_{2}(10 \mathrm{GPa})$, and we conclude that the magnetic transitions are fundamentally caused by the metallization. We find evidence in $\mathrm{NiI}_{2}$ and $\mathrm{CoI}_{2}$ that the $\mathrm{IM}$ transitions are first-order with a discontinuous closure of the charge-transfer gap. The difference between the $\mathbf{I M}$ transition pressure and the magnetic transition pressure in $\mathrm{NiI}_{2}$ further suggests the existence of a new antiferromagnetic-metallic phase between 17 and $19 \mathrm{GPa}$. Our results and conclusions are consistent with recent model calculations. The pressure properties of $\mathrm{NiI}_{2}, \mathrm{CoI}_{2}$, and $\mathrm{FeI}_{2}$ determined from this work are summarized in table 9.1.

These results are the first report of pressure-induced metallization of transitionmetal compounds where the mechanism of metallization has been identified as due to closure of the charge-transfer energy gap. Before this work and the introduction of the ZSA framework, these compounds were thought to be Mott insulators with $U$ determining the minimum transport gap. The theoretical proposal by ZSA that many of these compounds are in fact charge-transfer insulators has opened the possibility that motallization can be induced by closure of the charge-transfer gap. While the two types of IM transitions (charge-transfer and Mott) share many common features, we have shown that absorption measurements are capable of distinguishing them.

In the field of high-pressure physics there have been many reports of metallization by bandgap closure. In each of these cases no aromalies in the experimental results have been observed and the gap is assumed to close continuously. Our study shows that the fundamental energy gap can close discontinuously. Jur results are quantitatively 
explained theoretically by the spontaneous formation of a condensed phase of excitons when the gap is small resulting in a first-order transition.

There is further research to be done in this field. It is obviously desirable to obtain Mössbauer spectra on $\mathrm{FeI}_{2}$ and $\mathrm{x}$-ray measurements on $\mathrm{CoI}_{2}$ and $\mathrm{FeI}_{2}$. The magnetic properties such as magnetic susceptibility both in the insulating and metallic phases should be important for understanding the magnetic phase diagrams. The excitonic transitions also necessitate further study. If the cohesive energy of the condensed phase of excitons can be calculated for the three compounds, the value of the discontinuity in the absorption edge can be obtained for comparison with experimental results. Good agreement would be convincing evidence of an excitonic ground state in the metallic regime. In addition, the early-transition-metal iodides (e.g. $\mathrm{VI}_{2}$ and $\mathrm{TiI}_{2}$ ) also promise to be interesting because they are predicted to be true Mott insulators. The pressure dependence of $U$ is not known and a technique to measure it should be devised. It is expected that the charge-transfer gap will close faster than $U$ so that pressure-induced transitions from Mott insulators to charge-transfer insulators should be observable. 
Table 9.1 Summary of the properties of $\mathrm{NiI}_{2}, \mathrm{CoI}_{2}$, and $\mathrm{FeI}_{2}$ determined by this work

\section{Properties}

Metallization pressure determined by resistivity measurements (GPa)

$d E_{t} d P$ determined from temperature dependence of the resistivity (meV/GPa)

$d\left(E_{\mathrm{cTG}}\right) / d P \quad(\mathrm{meV} / \mathrm{GPa})$

Extrapolated $E_{\text {cTG }}$ closure pressure (GPa)

$\begin{array}{lll}\mathrm{NiI}_{2} & \mathrm{CoI}_{2} & \mathrm{FeI}_{2} \\ 17 & 1 \mathrm{C} & 23 \\ & \text { ref.[41] } & \end{array}$

$-110$

n.a.

n.a.

$-62$

$-82$

$-90$

21

17

22 


\section{References}

[1] N.F. Mott, Proc. Phys. Soc., London, Sect. A 62, 416 (1949), and Can. J. Phys. 34, 1356 (1956), and Philos. Mag. 6, 287 (1961).

[2] N.F. Mott, Metal-Insulator Transitions (Taylor and Francis, London, 1990).

[3] J. Hubbard, Proc. Roy. Soc. London, Ser. A 277, 237 (1964), and 281, 401 (1964).

[4] G.A. Sawatsky and J.W. Allen, Phys. Rev. Lett. 53, 2339 (1984).

[5] Jeffrey M McKay and Victor E. Henrich, Phys. Rev. Lett. 53, 2343 (1984).

[6] D.B. McWhan, J.P. Remeika, T.M. Rice, W.F. Brinkman, J.P. Maita, and A. Menth, Phys. Rev. Lett 27, 941(1971).

[7] F.J. Morin, Phys. Rev. Lett. 3, 34 (1959).

[8] J. Zaanen, G.A. Sawatzky, J.W. Allen, Phys. Rev. Lett. 55, 418 (1985).

[9] J. Zaanen, C. Westra, and G.A. Sawatzky, Phys. Rev. B 33, 8060 (1986).

[10] Y. Tokura, Y. Taguchi, Y. Okada, Y. Fujishima, T. Arima, K. Kumagai, Y. Iye, Phys. Rev. Lett. 70, 2126 (1993).

[11] J.B. Torrance, l?. Lacorre, and A.I. Nazzai, Phys. Rev. B 45, 8209 (1992).

[12] J.M. Mignot, G. Chouteau, G. Martinez, Phys. Rev. B 34, 3150 (1986).

[13] Kenneth A. Goettel, Jon. H. Egger, Isaac F. Silvera, Phys. Rev. Lett. 62, 665 (1989).

[14] H. K. Mao, R. J. Hemley, and M. Hanfland, Phys. Rev. Lett. 65, 484 (1990); Jon H. Eggert, Fred Moshary, William J. Evans, Hector E. Lorenzana, Kenneth A. Goettel, Issac F. Silvera, and William C. Moss, Phys. Rev. Lett. 66, 193 (1991).

[15] R.W.ن̀. Wyckoff, Crystal Structures, Vol I (Interscience, New York, 1963).

[16] Antônio J. R. da Silva and L.M. Falicov, Phys. Rev. B 45, 11511 (1992).

[17] Antônio J. R. da Silva, private communication.

[18] L.G. van Uitert, H. J. Williams, R.C. Sherwood, and J.J. Rubin, J. Appl. Phys. 36, 1029 (1965); H. Bizette, C. Terrier, and B. Tsai, C.R. Acad. Sci. 246, 
250 (1958); J.B. Goodenough, Magnetism and the Chemical Bond

(Interscience, New York, 1963).

[19] S.R. Kuindersma, J.P. Sanchez, and C. Haas, Physica 111B, 231 (1981).

[20] C.R. Ronda, G.J. Arends, and C. Haas, Phys. Rev. B 35, 4038 (1987).

[21] S. Antoci and L. Mihich, Phys. Rev. B 14, 5768 (1978); 21, 3383 (1980).

[22] H.I. Stamberg, M.T. Johnson, and H.P. Hughes, J. Phys. C 19, 2689 (1986).

[23] I. Pollini, J. Thomas, and A. Lenselink, Phys. Rev. B 30, 2140 (1984).

[24] S.R. Kuindersma, P.R. Boudewijn, and C. Haas, Phys. Stat. Sol. B 108, 187 (1981).

[25] M. Kozielski, I. Pollini, G. Spinolo, J. Phys. C 5, 1253 (1972).

[26] B.I. Halperin and T.M. Rice, Revs. of Mod. Phys. 40, 755. (1968).

[27] D.B. McWhan and T.M. Rice, Phys. Rev. Lett. 22, 887; D.B. McWhan, T.M. Rice, and P.H. Schmidt, Phys. Rev. 177, 1063.

[28] C. Kittel, Introduction to Solid State Physics (John Wiley and Sons, New York, 1986).

[29] R. Ramirez and L.M. Falicov, Phys. Rev. B 3 , 2425 (1971); A. Jayaraman, Phys. Rev. Lett. 29, 1674 (1972); A. Jayaraman, V. Narayanamurti, F. Bucher, and R.G. Maines, Phys. Rev. Lett 25, 368 and 1480 (1970).

[30] Andreas Giesekus and L.M. Falicov, Phys. Rev. B 44, 10449 (1991).

[31] J.K. Freericks and L.M. Falicov, Phys. Rev. B 45, 1896 (1992).

[32] A. Jayaraman, Rev. of Mod. Phys. 55, 65 (1983).

[33] D. Erskine, P. Y. Yu, and G. Martinez, Rev. Sci. Instrum. 58, 406 (1987).

[34] Anthony L. Chen, Steven P. Lewis, Zhenpeng Su, Peter Y. Yu, and Marvin L. Cohen, Phys. Rev. B 46, 5523 (1992).

[35] L. Merrill and W.A. Bassett, Rev. of Sci. Instr. 45, 290 (1974).

[36] J. A. Wolk, M.B. Kruger, J.N. Heyman, W. Walukiewicz, R. Jeanloz, and E. E. Haller, Semicond. Sci. Technol. 6, B78 (1991). 
[37] Moshe P. Pasternak, R. Dean Taylor, Anthony Chen, Charles Meade, L.M. Falicov, Andreas Giesekus, Raymond Jeanloz, and Peter Y. Yu, Phys. Rev. Lett. 65, 790 (1990).

[38] Moshe P. Pasternak, R. Dean Taylor, Raymond Jeanloz, J. Appl. Phys.70, 5956 (1992).

[39] Charles Meade and Raymond Jeanloz, private communication.

[39a] S. Morris, Proc. R. Soc. Lond. A 436, 203 (1992).

[40] Anthony L. Chen, Peter Y. Yu, and R. Dean Taylor, to be published.

[41] Eran Stener and Moshe P. Pastımak, High Pressure Research 1992 (Gordon and Breach Science Publishers '́.A., United Kingdom, 1992).

[42] T. Ishii, Y. Sakisaka, T. Matsukawa, S. Sato, and T. Sagawa, Sol. Sta. Comm., 13, 281 (1973).

[43] I. Pollini, G. Spinolo, G. Benedek, Phys. Rev. B 22, 6369 (1980).

[44] Walter A. Harrison, Electronic Structure and the Properties of Solids: The Physics of the Chemical Bond (W.H. Freeman and Company, San Francisco, 1980).

[45] H. G. Drickamer and C.W. Frank, Electronic Transitions and the High Pressure Chemistry and Physics of Solids (Chapman and Hall, London, 1973).

[46] Y.M. de Haan, Natl. Bur. Stand. (U.S.), Spec. Publ. 301, 233, (1969). 


\section{Appendix A: Pressure Dependence of the Intra $d$ Transitions}

In transition-metal compounds such as the transition-metal halides, the $3 d$ electrons are localized on the transition-metal ion. The energy levels in the solid arising from these localized $d$ states are not easily determined by band-structure calculations. It is a better approximation to treat the ion as a localized impurity state perturbed by the local crystal field of its nearest-neighbor ions. Since the crystal field imposes a symmetry that is less than the complete rotational symmetry of the free ion, the $3 d$ energy levels that were degenerate in the free ion are split into various levels. This simple theory was very successful explaining the observed spectroscopic lines in the IR and visible range of many insulators doped with transition-metal ions. These splittings are imposed solely by symmetry considerations.

Pressure can have two effects on the splittings of the $d$ levels within a crystal. First, pressure can strengthen the effect of the crystal field by decreasing the distance between the transition-metal ion and its nearest neighbor. Transitions between the split levels may shift in energy. For example, it is well known that an $O_{h}$ field splits the singleelectron, free ion $d$ levels into two degenerate levels, one with $T_{2 \mathrm{~g}}$ symmetry and the other with $E_{\mathrm{g}}$ symmetry [45]. The value of the splitting is conventionally denoted by $10 \mathrm{Dq}$. Pressure causes $10 D q$ to increase as $\left(r_{0} / r\right)^{5}$ where $r$ refers to the interatomic distance. This effect has been measured in systems where the transition-metal ion experiences a cubic crystal field (e.g. $\mathrm{MgO}$ doped with $\mathrm{Ni}^{2+}$ ) and calculated by molecular orbital theory. For a full treatment see ref. [45].

If, on the other hand, the symmetry is altered by application of an external parameter, the split levels of the transition-metal $3 d$ ion will shift and regroup in such a way as to reflect the new symmetry environment. For example, if one is somehow able to increase the lattice constants to infinity, the $3 d$ levels should asymptotically approach the free ion configuration. It is this second effect of pressure on the $d$ levels that we wish to study in $\mathrm{NiI}_{2}$ and $\mathrm{CoI}_{2}$. In these compounds the hexagonal crystal structure is completely 
determined by the $c$ and $a$ lattice constants and the angle $\chi$, defined as the angle made by the $c$-axis and a line joining a metal ion with any of its nearest neighbor iodine atoms (see Fig. A.1). For an arbitrary value of $\chi$, the point-group symmetry is trigonal $\left(D_{3 d}\right)$. However, when $\chi=x_{c}$ where $x_{c}$ is defined such that $\cos \left(x_{c}\right)=1 / \sqrt{3} \quad\left(x_{c}=54.7^{\circ}\right)$, the crystal field becomes cubic ( $O_{h}$ group) and the symmetry becomes higher. Extra symmetry operations become apparent. For example a $90^{\circ}$ rotation about the axis joining an $\mathrm{I}$ - ion with its nearest neighbor $\mathrm{Ni}^{2+}$ ion is normally not a symmetry operation when the symmetry is trigonal but becomes one when $\chi=\chi_{c}$. Under pressure, the value of $c$ decreases faster than the value of $a$, suggesting that $\chi$ will increase under pressure (Fig. Al). If $\chi<\chi_{c}$ at zero pressure and $\chi$ increases under pressure, the trigonal crystal field will pass through the point of higher, cubic symmetry at sufficiently high pressure. In the literature, the reported zero pressure value of $\chi$ for $\mathrm{CoI}_{2}$ is $54^{\circ}$ so that $\chi$ starts out less than $\chi_{c}[15]$. For $\mathrm{NiI}_{2}$ both $54^{\circ}\left(\chi<\chi_{c}\right)$ and $55.9^{\circ}\left(\chi>\chi_{c}\right)$ have been reported as zero pressure values $[15,46]$.

da Silva and Falicov (dSF) [16] have calculated the positions of the $3 d$ levels as a function of $\chi$ for $\mathrm{NiI}_{2}$ and $\mathrm{CoI}_{2}$. Because $\chi$ is almost equal to $\chi_{\mathrm{c}}$ in both materials, the crystal field can be decomposed into a strong cubic field plus a smaller trigonal distortion. In the free $\mathrm{Ni}^{2+}$ ion, the eight $d$ electrons have ${ }^{3} F$ symmetry. In $\mathrm{NiI}_{2}$ the ground-state representation of the $3 d$ electrons in the $O_{h}$ group is ${ }^{3} A_{2 \mathrm{~g}}$ and the first excited state has ${ }^{3} T_{2 \mathrm{~g}}$ symmetry. The trigonal distortion splits the ${ }^{3} T_{2 \mathrm{~g}}$ state into two states with ${ }^{3} e_{\mathrm{g}}$ and ${ }^{3} a_{1 \mathrm{~g}}$ representations of the $D_{3 d}$ point group (Fig. A2). Spin-orbit coupling is included in their calculations and accounts for the finer splittings. dSF's calculation of the energies of these levels in $\mathrm{NiI}_{2}$ as a function of $\chi$ are shown in Fig. A3. When $\chi<\chi_{c}$ the four lowest energy lines represent transitions from the ground state to the spin-orbit split ${ }^{3} e_{\mathrm{B}}$ states while the two highest energy lines are transitions from the ground state to the spin-orbit split ${ }^{3} a_{2 \mathrm{~B}}$ states. As $\chi$ increases these two groups of transitions approach each other. At the point $\chi=\chi_{c}$, states of the same symmetry from the two groups exhibit anti-crossing as 
a result of the disappearance of the trigonal field. States of different symmetry, such as $e$ and $a_{1}$, cross each other. Note that at values of $\chi>\chi_{c}$ the levels $e$ and $a_{2}$ cross also. Unlike the crossing at $\chi_{e}$, which is dictated by symmetry, the crossings at $\chi \neq \chi_{c}$ depend on the material parameters.

In $\mathrm{CoI}_{2}$ the cubic crystal field creates a ground state with representation ${ }^{4} T_{1 \mathrm{~g}}$ and a first excited state with representation ${ }^{4} T_{28}$ (Fig. A2). The trigonal crystal field and spinorbit coupling produce a manifold of states from the two representations. The transitions from ground state to the split ${ }^{4} T_{2 \mathrm{~g}}$ levels are shown as a function of $\chi$ in Fig. A4. As in $\mathrm{NiI}_{2}$ these transitions can be divided into two groups that come together and fan out again as $\chi$ passes through $\chi_{c}$.

Transitions within the $d$ states are dipole-forbidden but can be easily observed in crystals by involving phonon emissions and absorption [24,25]. The resultant phonon sidebands of these $d$-transitions are broad. These transitions in $\mathrm{NiBr}_{2}$ and $\mathrm{NiCl}_{2}$ measured at atmospheric pressure are shown in Fig. 2.4. Although in these crystals the halogen ions are different, the positions of the $d$ transitions are similar. Because the absorption peaks are broad, only the larger splittings can be seen. Our absorption measurements of $\mathrm{NiI}_{2}$ and $\mathrm{CoI}_{2}$ are displayed at the end of Chapter VII in Figs. 7.1 and 7.2 respectively. The positions of the broad intra-d transitions are marked with arrows. For $\mathrm{NiI}_{2}$, two overlapping peaks can be observed for the $0.0 \mathrm{GPa}$ spectrum. The spectrum shows excellent agreement with a higher-resolution, atmospheric pressure measurement in the literature [24]. At $2.6 \mathrm{GPa}$ the intra-d transitions become narrower, suggesting that the two peaks have merged. At $4.0 \mathrm{GPa}$ they split apart again; one moves toward higher energies while the other moves toward lower energies. Eventually at higher pressures, the absorption edge overlaps with these two peaks and makes it impossible to observe them. The absorption edge obscures both peaks as it shifts under pressure. However, between 5 and $10 \mathrm{GPa}$ the lower energy peak can still be discerned as a "pedestal" near the absorption edge. This "pedestal" is an additional feature that did not exist in the edge at 
$0.0 \mathrm{GPa}$. In contrast the $\mathrm{CoI}_{2}$ spectra show only a single peak that shifts toward higher energies under increasing pressure. There is no evidence of any sharpening or splitting of this peak under pressure. Again our atmospheric pressure measurement in $\mathrm{CoI}_{2}$ agrees very well with previous high-resolution experimental studies, which also show a single, unresolvable peak [24]. The peak positions of the intra-d transitions in both $\mathrm{NiI}_{2}$ and $\mathrm{CoI}_{2}$ are plotted in Fig. A5 as a function of pressure.

We interpret the behavior of the two intra-d transitions in $\mathrm{NiI}_{2}$ under pressure in terms of the two groups of transitions calculated by dSF. At zero pressure the two peaks have been assigned to the transitions from the ground state $\left({ }^{3} a_{2 \mathrm{~g}}\right)$ to the ${ }^{3} e_{\mathrm{g}}$ and ${ }^{3} a_{1 \mathrm{~g}}$ states of the trigonal crystal field. Because the transitions are broadened by phonon assistance, the individual spin-orbit split transitions cannot be resolved. Additional measurements were performed at $77 \mathrm{~K}$, but they did not show any significant sharpening of the transitions or any new structures. This is in agreement with the results on $\mathrm{NiCl}_{2}$ and $\mathrm{NiBr}_{2}$ (Fig. 2.4) reported by Kozielski et. al [25]. These authors have measured the ${ }^{3} T_{28}$ transitions of $\mathrm{Ni}^{2+}$ at temperatures down to $5 \mathrm{~K}$. They found no sharpening of the absorption peaks down to $77 \mathrm{~K}$ and only weak additional structures appearing at $5 \mathrm{~K}$. From our data in Fig. A5 it is impossible to tell whether the two peaks in $\mathrm{NiI}_{2}$ cross or anti-cross. Nevertheless the merging and separation of the two peaks under pressure is consistent with the theoretical calculations and suggests that in $\mathrm{NiI}_{2} \chi<\chi_{c}$ at atmospheric pressure and $\chi=\chi_{c}$ at about $2 \mathrm{GPa}$.

On the other hand, there is no experimental evidence of multiple peaks or crossings in the $\mathrm{CoI}_{2}$ data. The calculated values for the two groups of transitions do lie within the observed, broad single peak of width $-0.2 \mathrm{eV}$ at atmospheric pressure. Unlike $\mathrm{NiI}_{2}, \mathrm{CoI}_{2}$ has many low-lying states within $1000 \mathrm{~cm}^{-1}$ of the ground state that are thermally populated at $300 \mathrm{~K}$. Transitions from these states to the higher-energy levels can merge together with the calculated transitions from the ground state. If there were a cross-over, as predicted by theory, we would expect the overall width of the broad peak to decrease 
with pressure and then increase with pressure. We have seen only slight broadening of the absorption peak in $\mathrm{CoI}_{2}$. The results do not support a pressure-induced crossing at pressures less than $4.8 \mathrm{GPa}$ but also cannot rule out the possibility that crossings may occur within the broad peak.

In summary, a pressure induced crossing (or anti-crossing) of two intra-d transitions in $\mathrm{NiI}_{2}$ is observed by near-IR absorption measurements. They are consistent with calculations of the pressure dependence of such transitions by dSF, suggesting that at atmospheric pressure the trigonal crystal field of $\mathrm{NiI}_{2}$ corresponds to $\chi<\chi_{c}$ and that the field becomes cubic at a pressure of about $2 \mathrm{GPa}$. In contrast, the measurements of $\mathrm{CoI}_{2}$ clearly show only a single, unresolved peak that increases in energy with pressure. The latter result does not indicate that the point-group symmetry of $\mathrm{CoI}_{2}$ becomes cubic under pressure. 


\section{Figure Captions for Appendix A}

Fig. Al Proposed symmetry of the crystal field of the transition-metal halides under pressure. a) $\chi<\chi_{c}$, the point-group symmetry is $D_{3 d}$. b) $\chi=\chi_{c}: O_{h}$. c) $\chi>\chi_{c}: D_{3 d}$.

Fig. A2 Splitting of the $d$ levels of the $\mathrm{Ni}^{2+}$ and $\mathrm{Co}^{2+}$ ions by successive application of cubic, trigonal, and spin-orbit fields. Vertical arrows indicate transitions that occur in the near-IR

Fig. A3 Calculated crossings and anti-crossings of the near-IR intra-3d transitions in $\mathrm{NiI}_{2}$ as a function of $\chi$. From ref. [16].

Fig. A4 Calculated crossings and anti-crossings of the near-IR intra-3d transitions in $\mathrm{CoI}_{2}$ as a function of $\chi \cdot[16]$.

Fig. A5 Peak positions of the intra-3d transitions as a function of pressure. Lines are a least-squares fit to the data. 
a) $x<x_{c}$

b) $x=x_{c}$

c) $x>x_{c}$
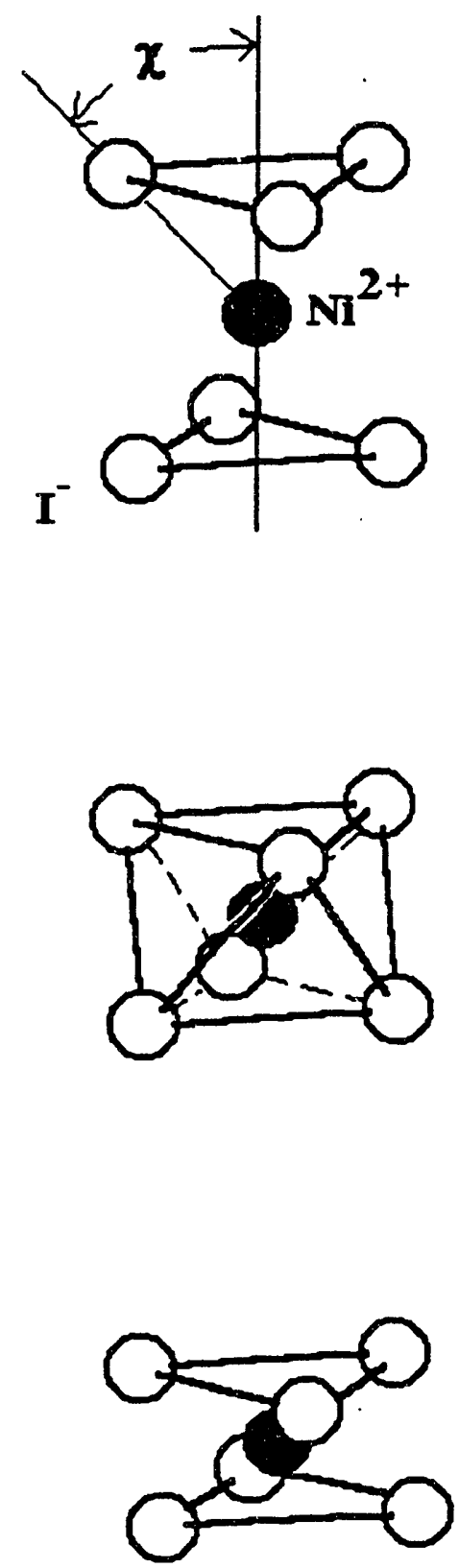

Fig. A1 
Free Cubic Trigonal Spin Orbit Ion Field Field
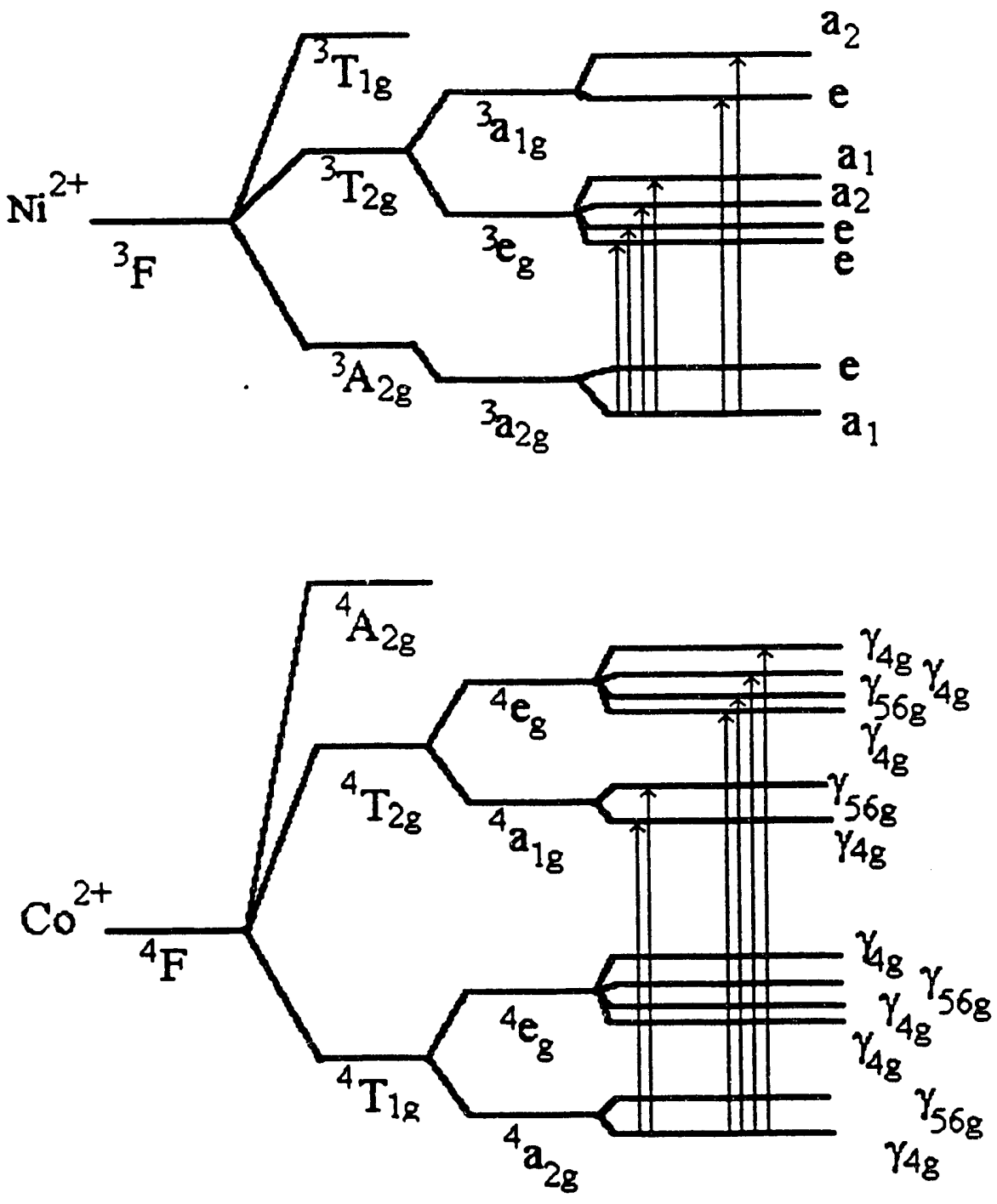

Fig. A2 


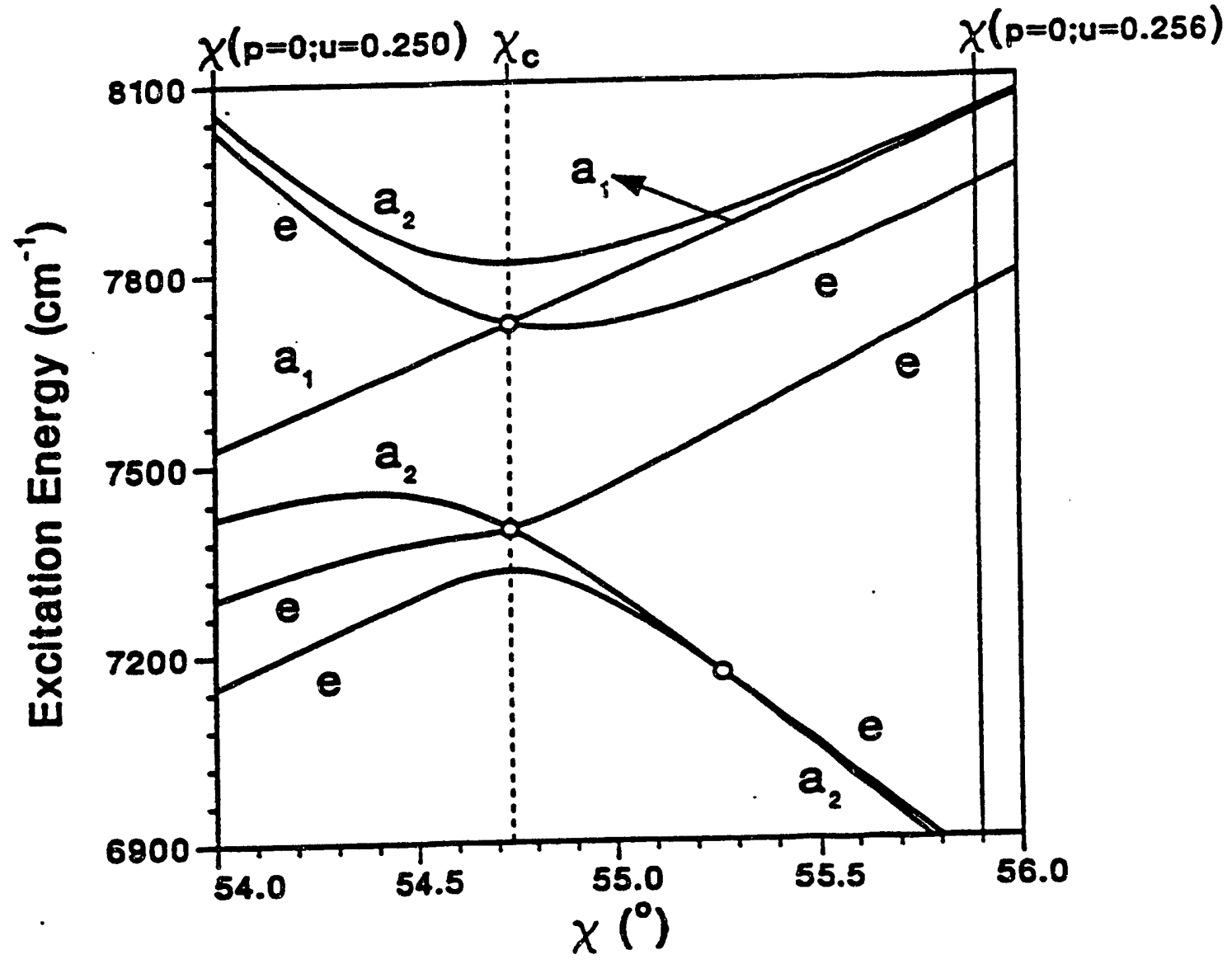

$\mathrm{NiI}_{2}$

Fig. A3 


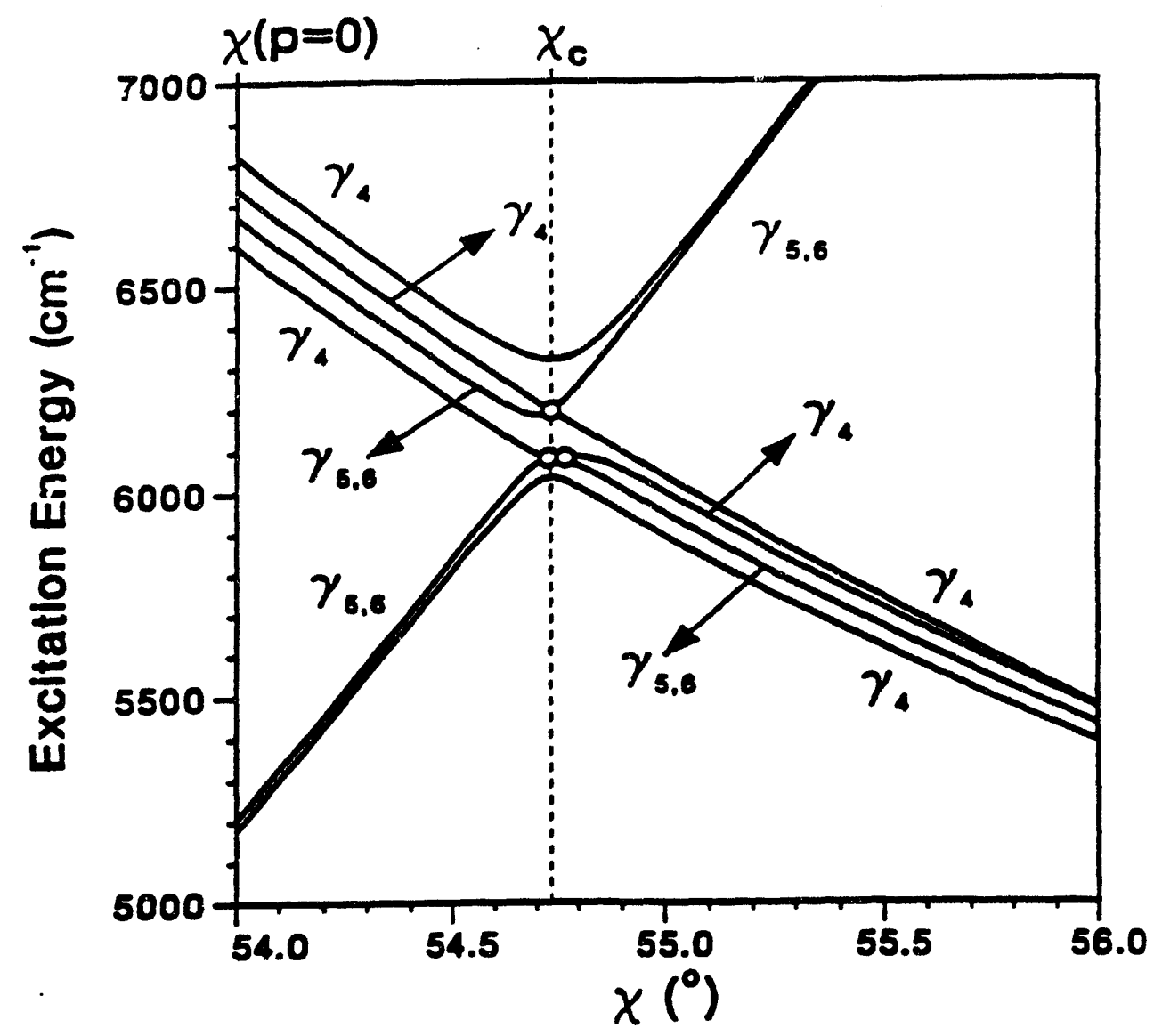

$\mathrm{CoI}_{2}$

Fig. A4 


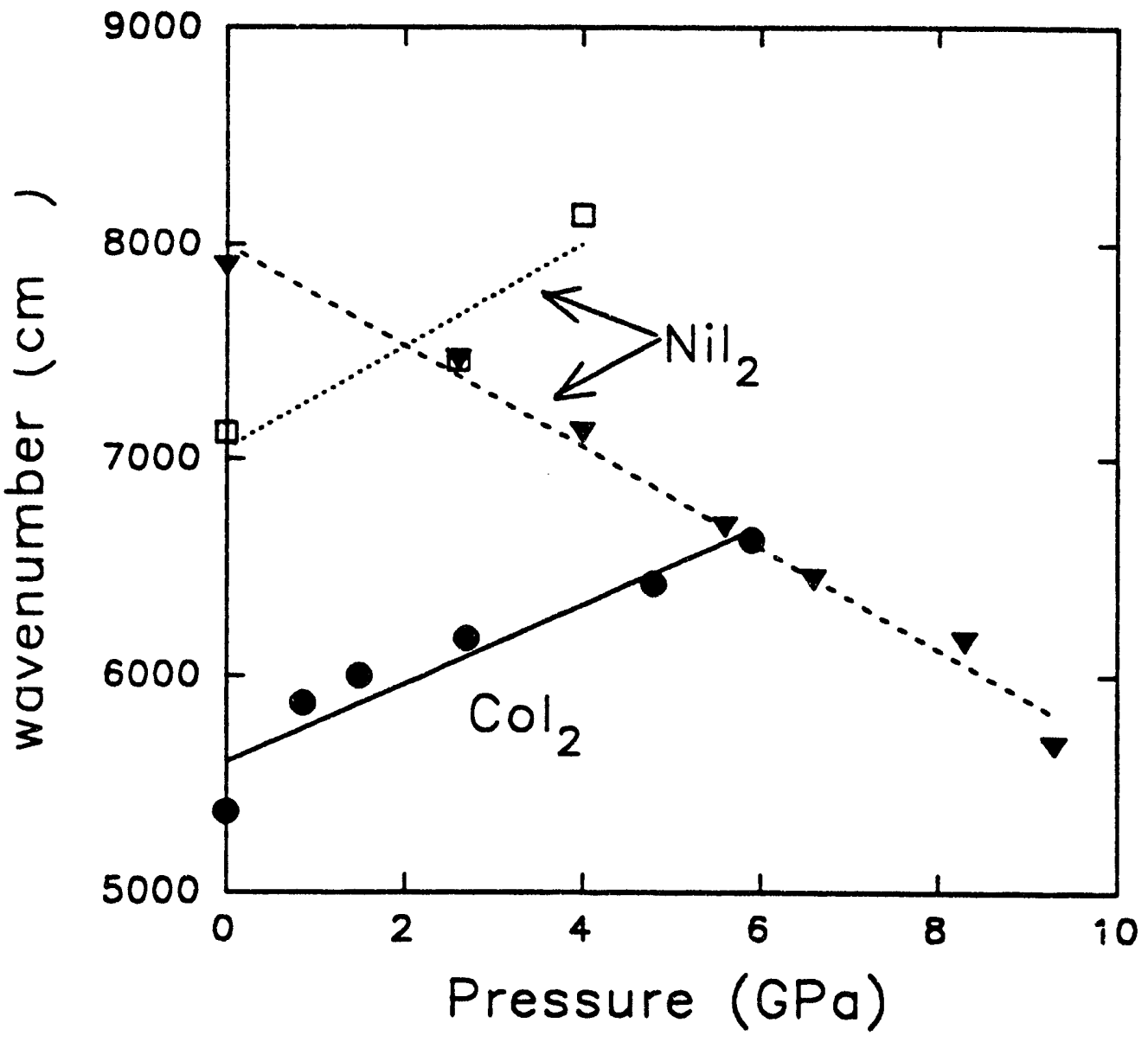

Fig. A5 

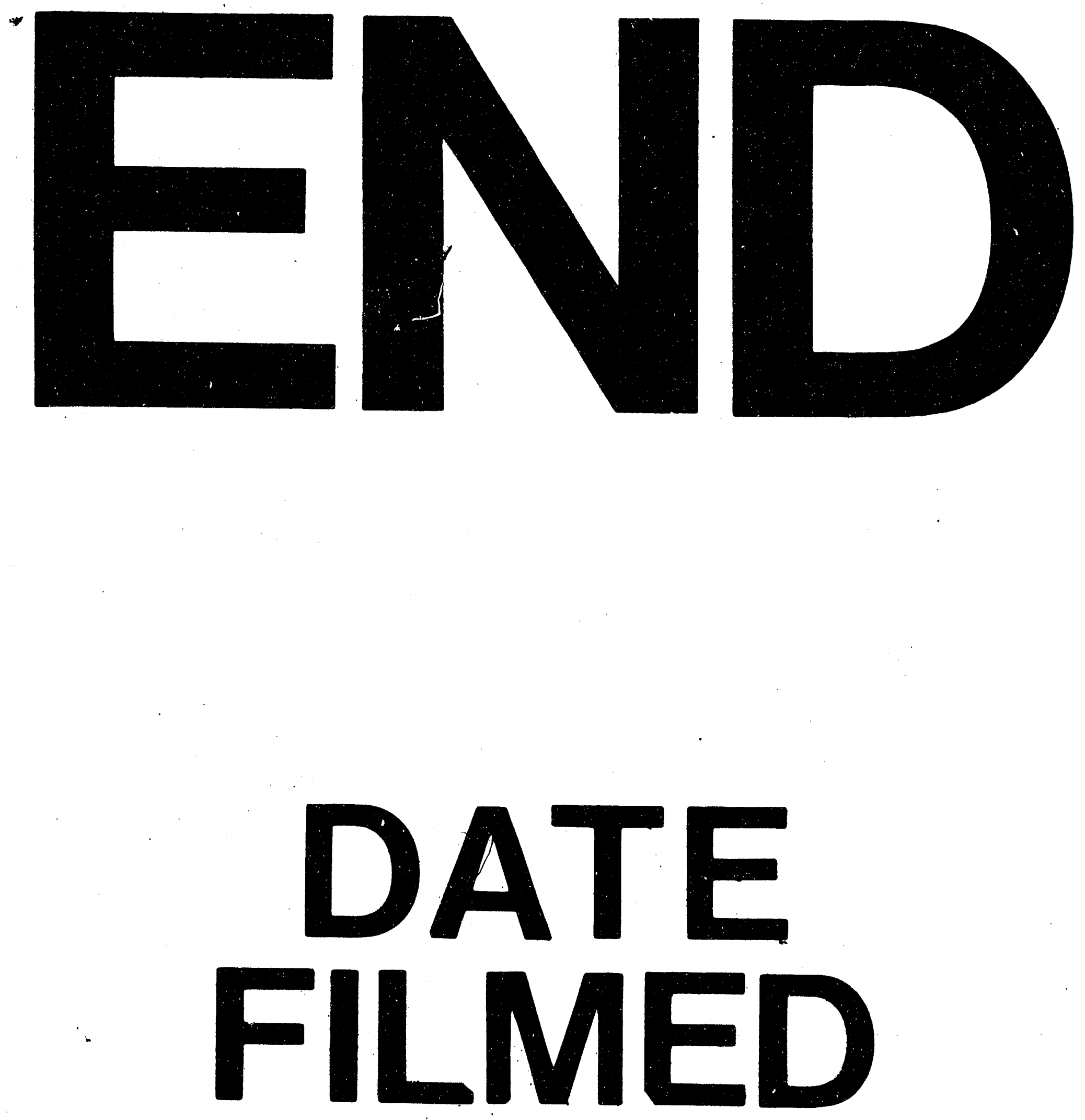

1

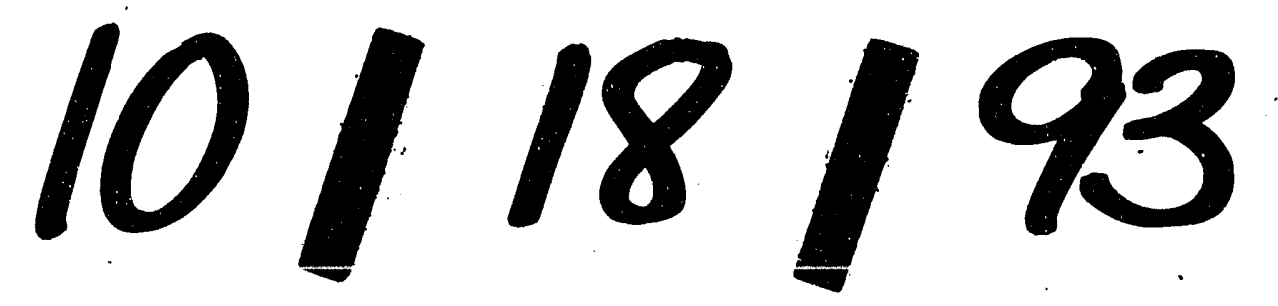


